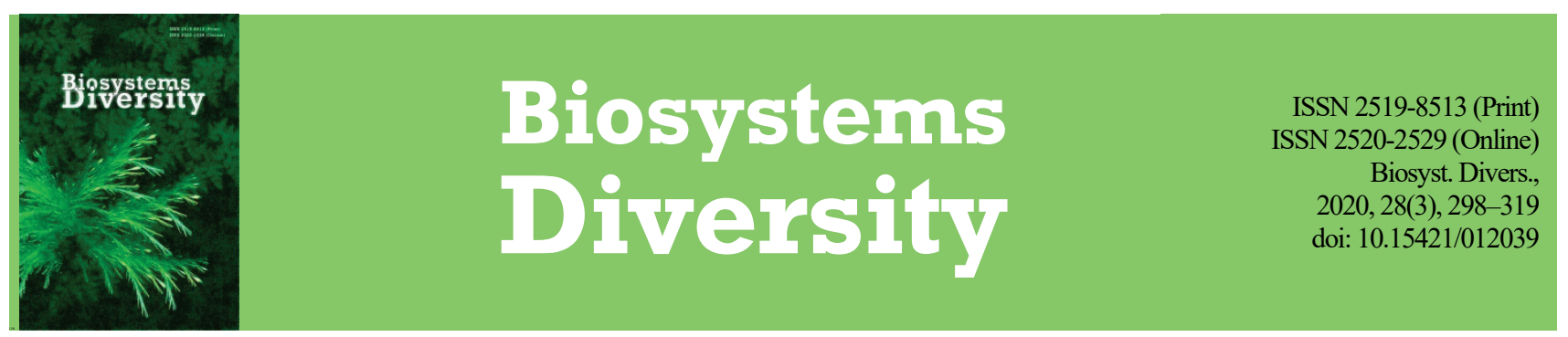

\title{
Syntaxonomy and ecological differentiation of the pioneer vegetation of Ukraine. 2. Helichryso-Crucianelletea maritimae, Festucetea vaginatae, Koelerio-Corynephoretea canescentis classes
}

\author{
D. V. Dubyna, T. P. Dziuba, S. M. Iemelianova, P. A. Tymoshenko \\ M. G. Kholodny Institute of Botany, National Academy of Sciences of Ukraine, Kyiv, Ukraine
}

Article info

Received 10.06.2020

Received in revised form 07.08 .2020

Accepted 09.08.2020

M. G. Kholodny Institute of Botany, NAS of Ukraine Tereshchenkivska st., 2 Kyiv, 01004, Ukraine Tel.: +38-099-079-01-08.

E-mail:ddub@ukr.net

\begin{abstract}
Dubyna, D. V., Dziuba, T. P., Iemelianova, S. M., \& Tymoshenko, P. A. (2020). Syntaxonomy and ecological differentiation of the pioneer vegetation of Ukraine. 2. Helichryso-Crucianelletea maritimae, Festucetea vaginatae, Koelerio-Corynephoretea canescentis classes. Biosystems Diversity, 28(3), 298-319. doi:10.15421/012039
\end{abstract}

Pioneer psammophytic vegetation is usually developed on wind-drift sandy substrates such as arenas, spits, beaches, river terraces, and this vegetation occupies significant areas in all three natural zones of Ukraine. The Koelerio-Corynephoretea canescentis class was represented by 13 associations, 3 alliances and 1 order; Festucetea vaginatae class by 22 associations, 2 alliances and 1 order; Helichryso-Crucianelletea maritimae by 10 associations, 4 alliances and 1 order. The results of cluster analysis and synoptic tables of the classes are presented. 9 alliances are briefly described. Leading factors of territorial and ecological differentiation are identified. It was found that the territorial distribution of plant communities is influenced by the character of ecotope mesorelief, soil composition and humus horizon thickness, as well as the degree of eolian processes development. The main factors of their ecological differentiation are soil acidity, salt regime and ombroregime. Based on the results of DCA-ordination of syntaxa within certain vegetation classes, it was found that their distribution is influenced by factors that correlate with the environment-specific conditions. It has emerged that an ecological differentiation of syntaxa within Festucetea vaginatae is determined by the integrated effect of gradients, and soil salinity is leading among them. Temperature regime and climate continentality are leading factors in the distribution of syntaxa within the Koelerio-Corynephoretea canescentis class. The gradients of ombroregime and soil humidity have a significant impact. The distribution of communities of the Helichryso-Crucianelletea maritimae class in the ecological space is determined mainly by factors of variability of damping, ombroregime and climate continentality. The author's syntaxonomic concept assumes the independence of the studied classes: Koelerio-Corynephoretea canescentis, Helichryso-Crucianelletea maritimae and Festucetea vaginatae, considering that the leading factors of community differentiation of the Festucetea vaginatae and Koelerio-Corynephoretea canescentis classes are the origin (genesis) of sandy substrates, as well as soil acidity. Phytosociological analysis of a large number of relevés of coastal littoral vegetation also provides support for independence of the Helichryso-Crucianelletea maritimae and Ammophiletea classes different floristically and ecologically. A review of the psammophytic vegetation of Ukraine will determine the place of the selected syntaxonomic units in the pan-European system.

Keywords: psammophytic vegetation; classification; new-formed ecotope; ecological gradients; cluster analysis; DCA-ordination.

\section{Introduction}

Pioneer psammophytic vegetation developed on wind-drift sandy substrates such as arenas, spits, beaches, terraces, etc., performs extremely important functions: biological, ecological, regulatory, recreational, and many others. It stabilizes loose scattered soils, creates preconditions for the development of vegetation in subsequent successional stages. The geocomplexes on which such vegetation occurs are sites of biological diversity formation and conservation. They are habitats for many plant and animal species, ways for dispersing their genetic material, and also serve as migration routes by which alien species spread actively. Newformed arena ecotopes are the centers of formation of endemic floristic complexes; they are characterized by generic endemism.

Syntaxonomy of psammophytic vegetation in Europe has been developed in sufficient detail. Classification schemes and characteristics were presented for the territories of the Czech Republic (Chytrý, 2007), Slovakia (Valachovič et al., 1995), Germany (Berg et al., 2004), Poland (Matuszkiewicz, 2008), France (Bardat et al., 2004), Romania (Sanda et al., 2008), Italy (Biondi et al., 2014), Spain and Portugal (RivasMartínez et al., 2001), Bulgaria (Tzonev et al., 2009), Hungary (Borhidi, 2003), and other countries. An overview of syntaxonomic units of the highest and middle levels in Europe is presented in generalized works: "Vegetation of Europe..." (Mucina et al., 2016), for the class Koelerio-
Corynephoretea canescentis - "Klasse Koelerio-Corynephoretea Klika in Klika et Novak 1941 - Sandtrockenrasen und Felsgrusfluren von der submeridionalen bis zur borealen Zone" (Dengler, 2004), HelichrysoCrucianelletea maritimae - "Classification of European and Mediterranean coastal dune vegetation" (Marcenò et al., 2018). However, discussions about syntaxonomic content and the structure of higher classification units of psammophytic vegetation are still ongoing.

The main areas of arenas in Ukraine are located in Polissia, and the smaller one in the forest-steppe zone. The largest continuous sand massifs in the steppe zone are located in the lower valley of the Dnieper River. This territory is called "Oleshkivsky Sands", and it occupies about 200 thousand hectares. Large areas are occupied by "Pridonetski Sands" (80 thousand ha) and sand areas on coasts, spits and islands of the Black and Azov Sea, Lower Danube and the Southern Bug River.

The macrorelief of sand massifs is mostly flat with small differences in elevation. However, the greatest importance in the distribution of plant communities belongs to the mesorelief of the sands, which is usually characterized by an alternation of high, up to 3-5 m, rounded (hills) or elongated dunes and fairly deep depressions. The topography and vegetation of wind-drift sands are mutually determining factors, since the absence of vegetation contributes to the dune formation, and their subsequent overgrowth promotes the formation of sand dunes, which over time are somewhat leveled in the hilly ones (Gordienko, 1969). 
Pioneer psammophytic vegetation is represented in Ukraine by three classes: Festucetea vaginatae, Helichryso-Crucianelletea maritimae and Koelerio-Corynephoretea canescentis. The syntaxonomy of vegetation of Festucetea vaginatae and Koelerio-Corynephoretea canescentis classes in Ukraine has been the subject of studies conducted by many authors. J. Vicherek on the territory of the lower and middle Dnieper River described Festucion beckeri alliance with 4 associations and, in fact, made an attempt to validate Festucetea vaginatae class, first identified by $R$. von Soó in 1968 (Vicherek, 1972). Secali-Stipetum borysthenicae association and two alliances: Verbascion pinnatifidi and Cynodonto-Teucrion polii were described for the coastal dune area in the Crimean Peninsula (Korzhenevsky, 1986; Korzhenevsky \& Klyukin, 1990). Arena landscapes of the "Biruchiy Island" spit in the Azov Sea were studied by Dubyna et al. (1995). On the territory of the spit, they identified 8 lowerrank syntaxa belonging to Festucetea vaginatae class, and two syntaxa attributed by the authors to Chenopodietea class. Three associations were described as new for science. Psammophytic vegetation of the Bilosaraiska Spit and the sea coast near Mariupol city was studied by Androsova \& Solomakha (1996). Didukh \& Korotchenko (1996) recorded a Centaureo borysthenicae-Festucetum beckeri association in the southern part of the forest-steppe zone of Ukraine, near the mouth of the Vorskla River and on islands of the Dniprodzerzhinsky and Pechenezhsky reservoirs. Umanets \& Solomakha (1999a, 1999b) conducted their research in the 1990s in the Central Black Sea region. In the Black Sea Biosphere Reserve, the vegetation syntaxonomy included without limitation psammophytic coenoses of Festucetea vaginatae class, within which the authors distinguished the Medicagini tenderiensis-Seselietalia tenderiensi order containing two alliances: Medicagini tenderiensisSeselion tenderiensi and Melico chrysolepi-Ephedrion distachyae. On the territory of the Danube Biosphere Reserve communities of 19 associations of the Festucetea vaginatae class were described (Dubyna et al., 1996, 2003). 11 associations of this class were identified on the territory of the National Nature Park "Dzharylhatskyi" (Dubyna \& Dziuba, 2005a; Davydova, 2019). In the Pryazovia spits: Obitochna, Berdianska, Kryva, Bezymenna, Shyrokinska, Bilosaraiska, Stepanivska and Fedotova, Tyshchenko (2006) recorded the distribution of communities of 9 associations belonging to the Festucetea vaginatae class; while three associations were newly identified in the course of surveys conducted by the author. In the estuarine region of the Dnieper River, Chynkina (2003) identified communities of one association. In addition to the southern regions, coenoses of the class were also observed in the Middle Dnieper River region, on islands of the Dnieper River within Kyiv city (Tsukanova, 2005; Aleshkina, 2011), as well as on the territory of the Kaniv Nature Reserve (Shevchyk \& Solomakha, 1996; Shevchyk et al., 1996) where the authors identified a Artemisio dniproicae-Salicion acutifoliae alliance. Its syntaxonomic status is debatable, since such communities involving shrubs are more often classified as Salicetea purpureae Moor 1958 (Mucina et al., 2016).

Phytocoenoses of sandy steppes were also observed in the northern steppe and forest-steppe zone of Ukraine, on the left bank of the Dnieper River (Bairak, 1998; Gomlya, 2005; Dziuba et al., 2010; Chusova, 2019); there the phytocoenoses occupy much smaller areas and are not characterized by coenotic diversity.

The syntaxonomic structure of the class has also been considered in generalized regional publications (Tymoshenko, 1999; Korzhenevsky et al., 2003; Dubyna et al., 2004; Solomakha, 2008; Dubyna et al., 2009).

Within the scope of final surveys on psammophytic vegetation in the Black Sea region, D. Dubyna and T. Dziuba in the "Prodrome of the vegetation of Ukraine" identified a new alliance Artemisio arenariaeFestucion beckeri in the Festucetea vaginatae class and isolated from one the class Helichryso-Crucianelletea maritimae, in which Ephedro distachyae-Medicaginetalia romanicae order was allocated for the Ukraine territory together with the alliance Ephedro distachyaeMedicaginion romanicae (Dubyna et al., 2019a). Unfortunately, the attempt of Korzhenevsky \& Kvitnitskaya (2014) to validate the alliances Verbascion pinnatifidi Korzhenevsky et Klyukin 1990 and CynodontoTeucrion polii Korzhenevsky et Klyukin 1990, as well as the vegetation associations of aeolian landforms in the Crimea, were not completely successful since expressis verbis "typus" should have been used to indi- cate the type of syntaxon name, according to article 5 of the ICPN (Weber et al., 2000).

Coenoses of the Koelerio-Corynephoretea canescentis class were first studied in Ukraine by Vicherek (1972) using the Braun-Blanquet approach on riverside sands of the Middle Dnieper River, where he described the Veronico dillenii-Corynephoretum association with its two subassociations. Later, coenoses belonging to this class (within the boundaries of Sedo-Scleranthetea and Festucetea vaginatae) were described for the Kaniv Nature Reserve area (Shevchyk \& Solomakha, 1996; Shevchyk et al., 1996). Didukh \& Korotchenko (1996) noted the distribution of plant communities belonging to the same association in the southern part of the left-bank forest-steppe zone of Ukraine, in the lower valley of the Vorskla River, in the upper valley of the Berestova River, and on islands of the Dniprodzerzhinsky and Pechenizhsky reservoirs. On left bank of the Dnieper River, coenoses belonging to this class were recorded by Bayrak (1998) and Galchenko (2006). V. Shevchyk and V. Solomakha, and later V. Shevchyk with O. Polishko described the communities of Thymo pallasiani-Centauretum sumensis and Cladonietum associations as part of the vegetation on the Dnieper River pine-forest terrace which grows along the bank of the Kaniv Reservoir (Shevchyk et al., 1996; Shevchyk \& Polishko, 2000; Polishko, 2001, 2005). On the Zhytomyr Polissia area, Yakushenko (2004) allocated the Artemisio campestris-Dianthetum borbasii association. Plant communities of the two associations were identified in area of the planned Korostyshivsky National Nature Park (Orlov \& Yakushenko, 2005). Coenoses of the Helichryso-Jasionetum association were described on the territory of the Polissky Nature Reserve (Vorobyov et al., 1998), and of Thymetum pulegioido-serpylli association were identified in the Cheremsky Nature Reserve (Konishchuk, 2006). Senchylo (2010) analyzed mainly coenoses of the KoelerioCorynephoretea canescentis class; the scientist performed phytocoenotic studies on 7 following plant associations in the Dnieper River floodplain area within the forest-steppe zone: Artemisio dniproicae-Sedetum sexangularis, Centaureo borysthenicae-Festucetum beckeri, Veronico dillenii-Secaletum sylvestri, Chamaecytiso ruthenici-Festucetum beckeri, Thymo pallasiani-Centauretum sumensis, Sedo sexangulare-Festucetum beckeri, Diantho borbasii-Agrostietum syreistschikovii. Among the syntaxonomic diversity of vegetation in the Pyryatinsky National Natural Park, Kovalenko (2016) identified communities of Veronico dilleniiSecalietum sylvestris, Linario odorae-Agropyretum dasyanthi and Chamaecytiso ruthenicae-Festucetum beckeri associations, which were assigned to the Festucetea vaginatae class. In Ukrainian Roztochia, Soroka (2008) identified Spergulo vernalis-Corynephoretum and Diantho-Armerietum associations.

Often plant communities of this class were described within SedoScleranthetea and, contrariwise, phytocoenoses associated with areas of poor skeletal soils and crystalline outcrops were sensu lato assigned to Koelerio-Corynephoretea canescentis class. Also, the structures of Festucetea vaginatae and Koelerio-Corynephoretea canescentis classes were sometimes intermixed (Shevchyk et al., 1996; Gaiova, 2015; Kovalenko, 2016). Regional publications summarized the study of the class in Ukraine (Onyshchenko, 2006; Solomakha, 2008; Dubyna et al., 2019a).

One of the generalizing works on classified syntaxa of xerophytic psammophytic vegetation in the forest and forest-steppe zones of the plain area of Ukraine was Kuzemko (2009) which includes syntaxonomic processing of 391 relevés. On the basis of cluster analysis and comparison of synoptic tables, the author identified 12 association-level syntaxa within Koelerio-Corynephoretea canescentis class (incl. Sedo-Scleranthetea and Festucetea vaginatae) and two associations within Molinio-Arrhenatheretea class. Phytosociological materials from Ukraine consisting of 179 relevés were included in the large-scale analysis of coastal dune vegetation in the Baltic-Atlantic, Mediterranean and Black Sea regions (Marcenò et al., 2018). The authors analyzed a large database (11,769 vegetation plots) and created the first formal classification of European coastal dune vegetation, accompanied by an expert system. This allowed them to identify 18 middle-rank syntaxa (alliances) which belong to the classes Ammophiletea, Honckenyo-Elymetea arenarii and KoelerioCorynephoretea canescentis, and providing a critical revision of the concept of the class Ammophiletea used in EuroVegChecklist. 
The issues on syntaxonomic structure and independence of KoelerioCorynephoretea canescentis, Festucetea vaginatae, and Helichryso-Crucianelletea maritimae classes are currently discussed. When they survey the areas where continental psammophytic steppes occupy significant areas and differ in phytocoenotic diversity, Ukrainian and Russian phytocoenologists are insistent on the independence of these classes (Demina, 2009, 2015; Dubyna et al., 2019a). Western European researchers more often adhere to a broad understanding of vegetation classes and suggest combining, in particular, Koelerio-Corynephoretea canescentis and Festucetea vaginatae (Mucina et al., 2016), as well as Ammophiletea and HelichrysoCrucianelletea maritimae (Marcenò et al., 2018). Phytocoenotic materials of the Sedo-Scleranthetea class very similar to Koelerio-Corynephoretea canescentis (Kuzemko, 2009; Kuzemko et al., 2014; Mucina et al., 2016) are subject to generalized analysis.

The analysis of significant databases of phytosociological relevés allows one to solve many issues on construction and effectiveness of vegetation classification (Lengyel et al., 2018; Willner et al., 2019; Bondareva et al., 2019; Landucci et al., 2020), biodiversity definition (Sabatini et al., 2018), biogeographic distribution of vegetation and its ecological affinity (Chytrý et al., 2019), and others. Currently, we have collected more than 1700 phytosociological relevés of psammophytic vegetation in Ukraine which includes more than 1000 relevés belonging to the classes Festucetea vaginatae, Helichryso-Crucianelletea maritimae, Koelerio-Corynephoretea canescentis, syntaxonomic processing of which, in our opinion, will make a certain contribution to studying of psammophytic vegetation in Europe and supplement its syntaxonomy.

In recent years, the ecological component of phytocoenoses has become increasingly used as an object of study and in classification proving (Çoban \& Willner, 2019; Zhou et al., 2019; Willner et al., 2019), including for the study of vegetation dynamics (Cao et al., 2019), identifying the main ecological factors of plant communities' differentiation (Kuzemko et al., 2016; Korolyuk et al., 2018), assessing the impact of environment on the floristic richness in phytocoenoses (Jansons et al., 2016; Yousaf et al., 2016; Slezák et al., 2017). The method developed by Didukh (2012) for determining the main eco-factors of the distribution of plant communities by 12 gradients completes phytosociological studies of vegetation and allows us to determine the ecosystem state by indicators of their biotic components.

The purpose of the paper was to generalize the accumulated phytocoenotic materials and existing data on syntaxonomy of the pioneer psammophytic vegetation in Ukraine, to clarify its current state and to develop a syntaxonomic structure, to establish the leading factors of ecological differentiation of phytocoenoses on the basis of ordination analysis, as well as to discuss controversial issues related to the syntaxonomic content of the classes Festucetea vaginatae, Koelerio-Corynephoretea canescentis, Helichryso-Crucianelletea maritimae.

\section{Materials and methods}

The work is based on the materials of field studies carried out by the authors during 1984-2018 according to the Braun-Blanquet approach to floristic classification (Braun-Blanquet, 1964; Westhoff \& van der Maarel, 1973). The standard size of plots was $4 \times 4 \mathrm{~m}$. Sometimes, in particular on elongated coastal zones and dunes, the plots have size $1 \times 4$ or $2 \times 5 \mathrm{~m}$. At the same time, requirements for structure uniformity of vegetation cover were met. Phytosociological analysis also included the relevés presented in abovementioned publications of J. Vicherek, O. Tyshchenko, Y. Didukh, I. Korotchenko, O. Senchylo, O. Umanets, I. Solomakha, V. Solomakha, A. Androsova, O. Orlova, D. Iakushenko, V. Shevchyk, O. Polishko, O. Bayrak, N. Galchenko, M. Soroka, A. Davydova, L. Gomlya, T. Chinkina, V. Korzhenevsky and A. Klyukin, and others. The database also included unpublished relevés of psammophytic vegetation from manuscripts and dissertations, kindly provided to us by A. Kuzemko, O. Kovalenko, O. Chusova, O. Senchylo, V. Kolomiychuk, T. Fitsailo, I. Goncharenko, V. Konishchuk, G. Tsukanova, Y. Gayova, D. Iakushenko, V. Konogray, I. Khomyak. The size of plots was not always specified in the relevés, but we used all the available ones.

The materials were ordered by development of a database of geobotanical relevés in TURBOVEG 2.79 (Hennekens \& Schaminée, 2001). Interpretation of the phytosociological material was carried out in several steps. At the initial one, the entire database of geobotanical relevés of halophytic, psammophytic and littoral vegetation in Ukraine (7388 relevés) was processed and divided into smaller groups based on their floristic differences using the method of two-way indicator species analysis (TWINSPAN), in particular its modified algorithm (Hill, 1979; Tichý, 2002; Roleček et al., 2009) implemented in the JUICE 7.0 software package. "Pseudospecies" cut level was $0 \%, 5 \%, 15 \%$ and $30 \%$. The Whittaker's beta was chosen as cluster heterogeneity measure (Whittaker, 1978). Further, the clusters were selected which corresponded to the classes of psammophytic vegetation, based on diagnostic species characteristics.

Since a significant number of relevés included only vascular plants, or when mosses and lichens were identidied only up to their genus, authors excluded mosses, lichens and algae species from processing in order to "align" the relevés. These species were added again at the final stages of compiling the phytocoenotic tables. Cluster analysis was carried out using the PC-ORD program after the rejection of a certain part of relevés related to Ammophiletea Br.-Bl. et Tx. ex Westhoff et al. 1946, Salicetea purpureae Moor 1958 (Artemisio dniproicae-Salicetum acutifoliae Shevchyk et Solomakha 1996), Pyrolo-Pinetea sylvestris Korneck 1974, Sedo-Scleranthetea Br.-Bl. 1955, relevés of ruderal vegetation, relevés attributed by the authors to communities, and also not containing dominant species at all. The Sörensen coefficient (Sörensen, 1948) was chosen as the similarity measure and grouping was performed using the "flexible" beta method at -0.25 . This resulted in finding smaller groups approximately corresponding to the association's rank. Relevés of Festucetea vaginatae class were grouped according to Ward's method (Ward, 1963). Identification of diagnostic species in syntaxa was carried out in accordance with the fidelity index - the Phi coefficient (Willner et al., 2009), the threshold values of which were assumed at the level of 25 . All relevé groups were standardized to equal size, and non-essential fidelity values were removed based on the Fisher exact test.

The taxonomic nomenclature was given by "Flora Europaea" (Tutin et al., 1964-1980). Some of the species (taxonomically problematic, narrow, considered differently by various authors) we have combined into aggregates. Specifically, Medicago sativa ssp. falcata agg. combined the species M. kotovii Wissjul., M. romanica Prodán, M. tenderiensis Opperman ex Klokov and actually M. falcata L. Crepis tectorum agg. includes $C$. tectorum L. and C. ramosissima D'urv. Artemisia campestris agg. combines $A$. campestris L., A. marschalliana Spreng. and $A$. dniproica Klokov.

Identification of the obtained phytocoenoses was carried out on the basis of their diagnostic species and floristic composition by comparison with foreign and Ukrainian publications (Demina, 2009, 2015; Mucina et al., 2016; Bulokhov, 2019; Dubyna et al., 2019a).

The method of DCA-ordination (Hill \& Gauch, 1980; ter Braak \& Smilauer, 2015) of the R-project software (Venables \& Smith, 2008) through the JUICE software package was used to determine the features of ecological differentiation of communities. The calculation of environmental parameters was carried out according to phytoindication scale developed by Didukh (2011), which allows ordination analysis to be performed on 12 factors: soil humidity $(\mathrm{Hd})$, variability of damping $(\mathrm{fH})$, soil aeration $(\mathrm{Ae})$, soil nitrogen content $(\mathrm{Nt})$, soil acidity $(\mathrm{Rc})$, salt regime $(\mathrm{Sl})$, carbonate content $(\mathrm{Ca})$, temperature regime $(\mathrm{Tm})$, ombroregime $(\mathrm{Om})$, climate continentality $(\mathrm{Kn})$, cryoregime $(\mathrm{Cr})$ and light intensity $(\mathrm{Lc})$.

\section{Results}

Cluster analysis of psammophytic vegetation database includes 1785 relevés and allowed several classes to be identified (Fig. 1). In particular, clusters 1-4 corresponded to the class Salicetea purpureae (Artemisio scopariae-Tamaricion ramosissimae), 5-12 - Ammophiletea, 13-15 Cakiletea maritimae, 16-20 and 32-35 - Helichryso-Crucianelletea maritimae, 21-31 - Koelerio-Corynephoretea canescentis, 36-41 and 49-70 - Festucetea vaginatae, 42-48 - Stellarietea mediae (ruderal vegetation on sandy soils).

At the next stage of processing, after exclusion of relevés related to Ammophiletea, Salicetea purpureae, Pyrolo-Pinetea sylvestris, relevés of disturbed habitats, as well as those that do not contain dominant species, phytocoenons with the studied classes remained: Helichryso-Crucianelletea maritimae, Koelerio-Corynephoretea canescentis and Festucetea vaginatae - 955 relevés (Fig. 2). Then we performed cluster analysis 
separately per classes. It should be noted that a significant number of sample sites within the studied classes were identified by us as agglomerative groups representing the initial stages of sand overgrowth, or as rankless (basal/derrivate) communities (a total of 258 relevés), which were not included by the authors at this stage in the classification scheme.

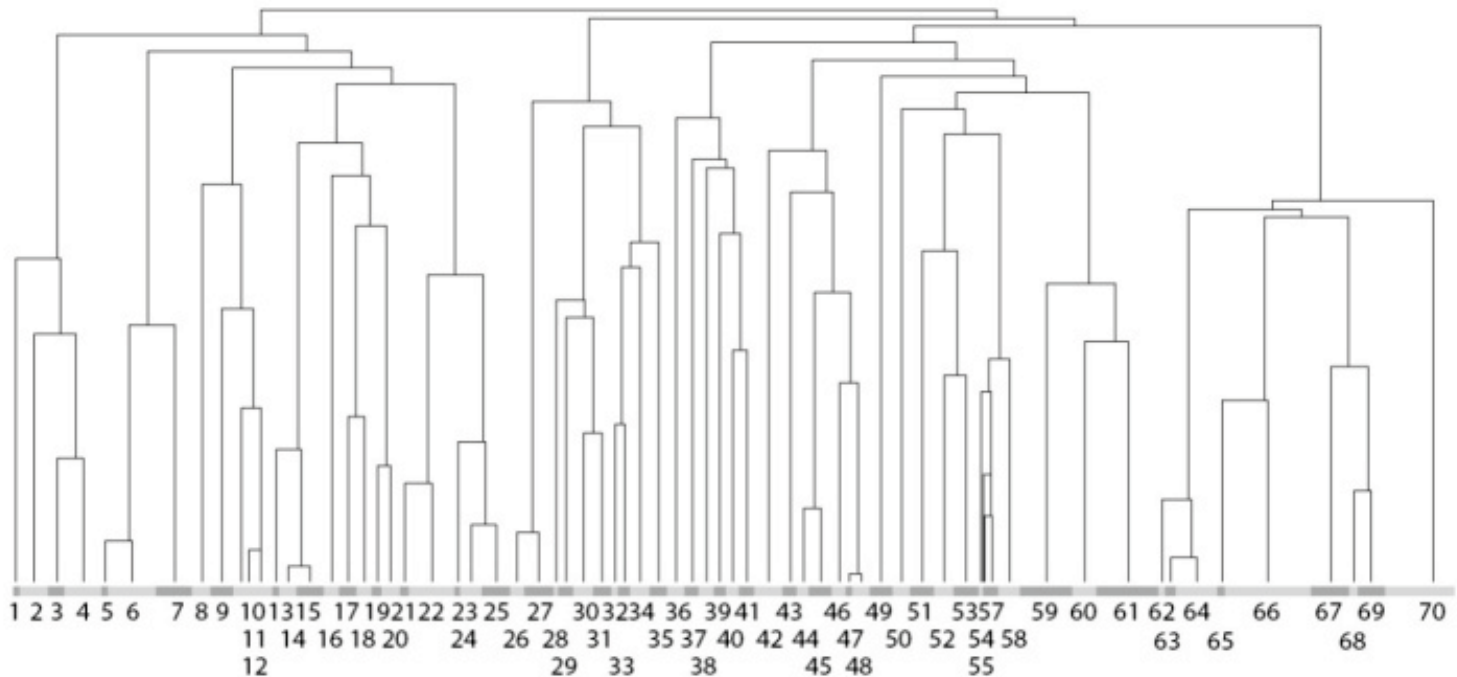

Fig. 1. Hierarchical cluster classification of relevés of psammophytic vegetation of Ukraine (1785 relevés) (PC-ORD)

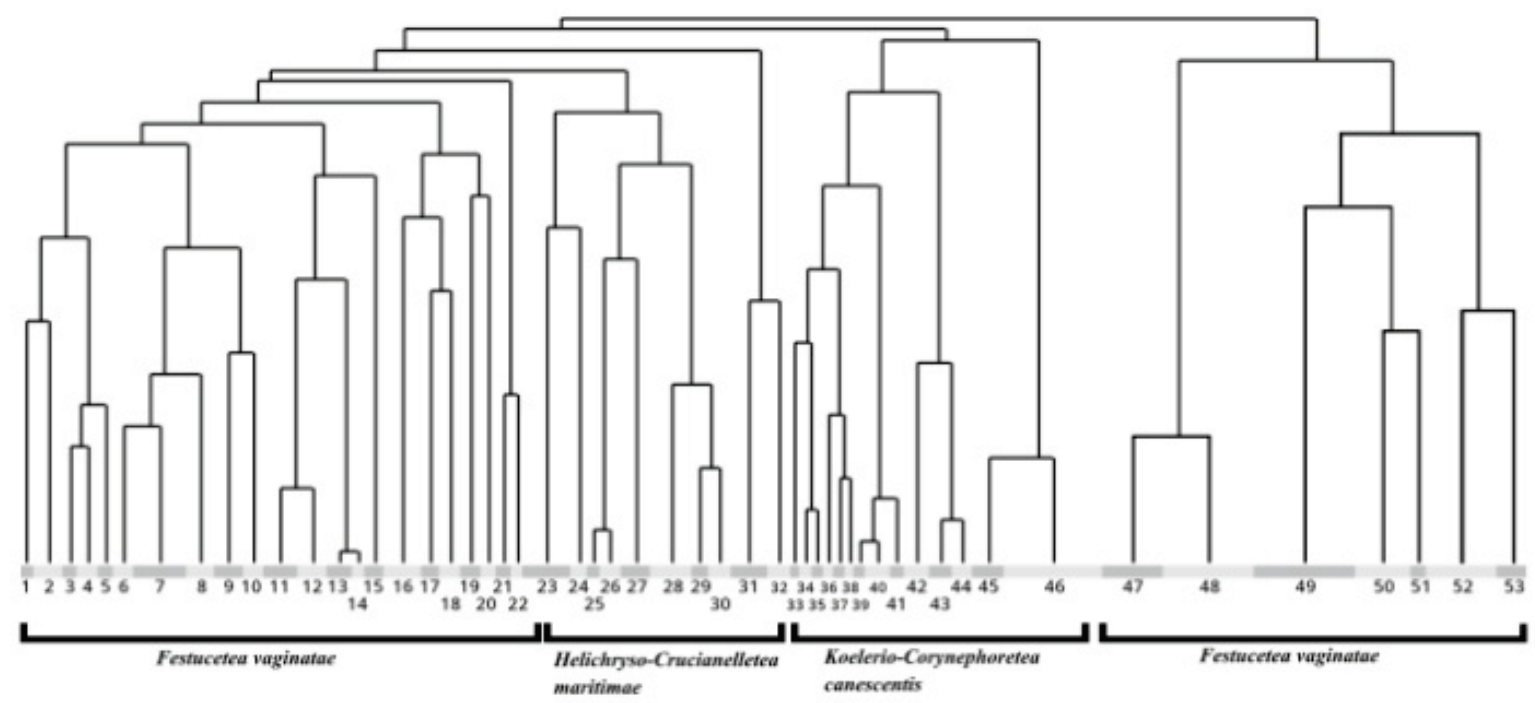

Fig. 2. Hierarchical cluster classification of psammophytic vegetation relevés of Ukraine (955 relevés) (PC-ORD)

Based on processing of phytocoenotic tables and cluster analysis results, a refined classification scheme of psammophytic vegetation of Ukraine was obtained.

Classification scheme of psammophytic vegetation of Ukraine Koelerio-Corynephoretea canescentis Klika in Klika et Novák 1941 Corynephoretalia canescentis Klika 1934

Corynephorion canescentis Klika 1931

Veronico dillenii-Corynephoretum Passarge 1960

Corniculario aculeatae-Corynephoretum canescentis Steffen 1931 (incl. Helichryso-Jasionetum sensu Vorobyov et al. 1998 non Libbert 1940) Armerion elongatae Potsch 1962

Diantho deltoidis-Armerietum elongatae Krausch 1959

Koelerion glaucae Volk 1931

Veronico dillenii-Secaletum sylvestris Shevchyk et Solomakha 1996

Chamaecytiso ruthenicae-Festucetum beckeri Shevchyk etal. 1996

Jasiono montanae-Thymetum serpylli Bulokhov 2019 (Thymetum

pulegioido-serpylli Konishchuk 2003 nom. inval. (syntax. syn.))

Jasiono montanae-Festucetum ovinae Klika 1941

Corynephoro-Silenetum tataricae Libbert 1931

Artemisio campestris-Dianthetum borbasii Yakushenko 2004

Artemisio dniproicae-Sedetum sexangularis Shevchyk et Solomakha in

Shevchyk et al. 1996 (incl. Sedo sexangulare-Festucetum Solomakha,

Shevchyk et Senchylo 1997 (art. 10); Poetum bulbosae Shevchyk et

Polishko 2000 (syntax. syn.))
Diantho borbasï-Agrostietum syreistschikovï Vicherek 1972

Thymo angustifolii-Festucetum beckeri Vicherek 1972

Centaureo borysthenicae-Festucetum beckeri Vicherek 1972

Festucetea vaginatae Soó exVicherek 1972

Festucetalia vaginatae Soó 1957

Artemisio arenariae-Festucion beckeri Dubyna et Dziuba in Dubyna et al. 2019 Aperetum maritimae Popescu, Sanda et Doltu 1980 (incl. Apero maritimiChrysopogonetum grylli Davydova 2019p.p. (syntax. syn.)

Danco guttati-Chrysopogonetum grylli Popescu, Sanda et Doltu 1980 (incl. Apero maritimi-Chrysopogonetum grylli Davydova 2019 (syntax. syn.))

Carici colchicae-Holoschoenetum vulgaris Sorbu et al. 1995

Salici rosmarinifoliae-Holoschoenetum vulgaris Mititelu et al. 1973 (incl. Galio ruthenici-Salicetum rosmarinifoliae I. Solomakha, Vorobyov et Moysienko 2015 nom. inval. (syntax. syn.))

Allio guttati-Festucetum rupicolae Umanets et Solomakha 1999

Heliotropio dolosi-Brometum japonici Dubyna, Neuhäuslová et ShelyagSosonko 1995

Trago-Anthemietum ruthenicae Pușcaru-Soroceanu etal. 1963

Plantaginetum arenariae (Buia etal. 1960) Popescu et Sanda 1987

Linario odorae-Agropyretum dasyanthi Vicherek 1972

Cynodonto-Medicaginetum minimae Popescu et Sanda 1975

Secali-Cynodontetum dactyli Dubyna, Neuhäuslová et Shelyag-Sosonko 1995

Secali-Stipetum borysthenicae Korzhenevsky ex Dubyna, Neuhäuslová et Shelyag-Sosonko 1995 
Secaletum sylvestris Popescu et Sanda 1973

Secali sylvestri-Brometum tectorum Hargitai 1940

Centaureo odessanae-Caricetum colchicae Tyschenko 1999 (incl. Secali sylvestri-Caricetum colchicae Davydova 2019 (syntax. syn.))

Centaureo odessanae-Stipetum capillatae Dubyna, Neuhäuslová et Shelyag-Sosonko 1995

Poo bulbosae-Caricetum colchicae Dubyna, Neuhäuslová et ShelyagSosonko 1995

Achilleo setacei-Festucetum beckeri Chusova 2019 nom. inval.(art. 3b)

Centaureo gerberi-Chamaecytisetum borysthenici Demina 2009

Festucion beckeri Vicherek 1972

Festucetum beckeri Ad. Oprea 1998

Centaureo odessanae-Festucetum beckeri Vicherek 1972

Centanreo brevicipiti-Festucetum beckeri Vicherek 1972

Helichryso-Crucianelletea maritimae Géhu et al. in Sissingh 1974

Ephedro distachvae-Medicaginetalia romanicae Dubyna et Dziuba in Dubyna et al. 2019 (incl. Medicagini tenderiensis-Seselietalia tenderiensi Umanets et I. Solomakha 1999 (syntax. syn.))

Ephedro distachyae-Medicaginion romanicae Dubyna et Dziuba in

Dubyna et al. 2019

Anisantho tectorum-Medicaginetum kotovii Tyschenko 1996

Anisantho tectorum-Helichrysetum arenariae Tyschenko 1999

Ephedro-Caricetum colchicae (Prodan 1939) Sanda et Popescu 1973

Scabiosion ucranicae Sanda et al. 1980

Scabioso ucranicae-Caricetum ligericae (Simon 1960) Krausch 1965

Secali sylvestri-Alyssetum borzaeani (Borza 1931) Morariu 1959

Medicagini tenderiensis-Seselion tenderiensi Umanets et I. Solomakha

1999 (incl. Melico chrysolepi-Ephedrion distachyae Umanets et I. Solomakha 1999 (syntax. syn.))

Medicagini tenderiensis-Seselietum tenderiensi Umanets et I. Solomakha 1999 (incl. Sileno subconicae-Galietum tenderiensi Umanets et I. Solomakha 1999 (syntax. syn.); Elytrigio elongataeGalietum tenderiensi Umanets et I. Solomakha 1999 (syntax. syn.); Consolido paniculatae-Anchusetum gmelini Umanets et I. Solomakha 1999 (syntax. syn.))

Cynodonto-Teucrion polii (Korzhenevsky et Klyukin 1990) Korzhenevsky et Kvitnytskaya 2014 nom. inval. (art. 5) (incl. Verbascion pinnatifidi Korzhenevsky et Klyukin 1990 (syntax. syn.))

Cynodonto-Ajugetum chiae (Korzhenevsky et Klyukin 1990)

Korzhenevsky et Kvitnytskaya 2014 nom. inval. (art. 5)

Carici liparicarpo-Centaureetum adpressae (Korzhenevsky et

Klyukin 1990) Korzhenevsky et Kvitnytskaya 2014 nom. inval. (art. 5)
Astragalo borysthenici-Ephedretum distachyae (Korzhenevsky et Klyukin 1990) Korzhenevsky et Kvitnytskaya 2014 nom. inval. (art. 5) Leymo-Verbascetum pinnatifidi (Korzhenevsky et Klyukin 1990) Korzhenevsky et Kvitnytskaya 2014 nom. inval. (art. 5)

Koelerio-Corynephoretea canescentis class includes pioneer communities on fluvioglacial and riverine sandy, shallow, weakly mobile soils, mostly dry and poor in nutrients, having acidic reaction. According to our results, the class in Ukraine include 1 order, 3 alliances and 13 associations (Fig. 3, Table 1).

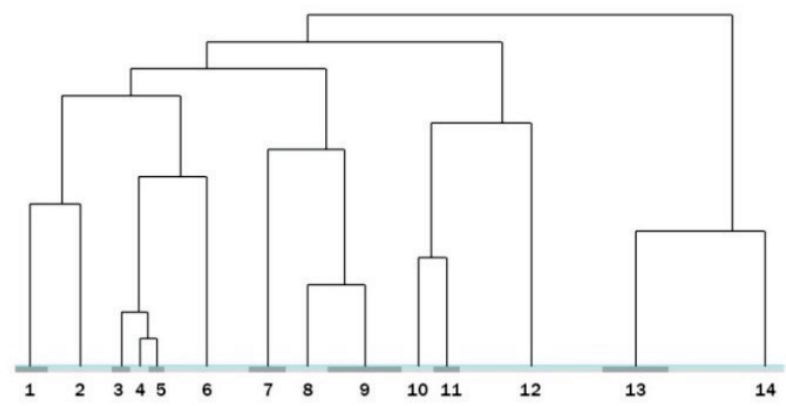

Fig. 3. Cluster analysis of phytosociological data of KoelerioCorynephoretea canescentis class: clusters: 1 - Veronico dilleniiSecaletum sylvestris association; 2 -Chamaecytiso ruthenicae-Festucetun beckeri; 3 -Diantho deltoidis-Armerietum elongatae;

4 -Jasiono montanae-Thymetum serpylli; 5 -Jasiono montanae-

Festucetum ovinae; 6-Corynephoro-Silenetum tataricae; 7 -Artemisio campestris-Dianthetum borbasii; 8-Artemisio dniproicae-Sedetum sexangularis; 9-Veronico dillenii-Secaletum sylvestris (sensu

Kovalenko); 10 - Diantho borbasii-Agrostietum syreistschikovii; 11 -

Thymo angustifolii-Festucetum beckeri; 12 - Centaureo borysthenicae-

Festucetum beckeri; 13 - Veronico dillenii-Corynephoretum;

14 -Corniculario aculeatae-Corynephoretum canescentis

Table 1

Synoptic table of the class Koelerio-Corynephoretea canescentis

\begin{tabular}{|c|c|c|c|c|c|c|c|c|c|c|c|c|c|c|}
\hline No. of syntaxa & 1 & 2 & 3 & 4 & 5 & 6 & 7 & 8 & 9 & 10 & 11 & 12 & 13 & 14 \\
\hline Number of relevés & 14 & 24 & 7 & 7 & 38 & 6 & 8 & 44 & 12 & 9 & 25 & 29 & 15 & 83 \\
\hline Aristolochia clematitis & 42.4 & - & - & - & - & - & - & - & - & - & - & - & - & - \\
\hline Tragopogon ucrainicus & 38.7 & - & - & - & - & - & - & - & - & - & - & - & 15.3 & - \\
\hline Vicia tetrasperma & 32.3 & - & - & - & - & - & - & - & - & - & - & - & - & - \\
\hline Cardaminopsis arenosa & 25.8 & - & - & - & - & - & - & - & - & - & - & - & - & - \\
\hline Salix acutifolia & 25.8 & - & - & - & - & - & - & - & - & - & - & - & - & - \\
\hline Galium ruthenicum & 25.8 & - & - & - & - & - & - & - & - & - & - & - & - & - \\
\hline Peucedanum oreoselinum & - & 64.1 & - & - & 19.3 & - & - & - & - & - & - & - & - & - \\
\hline Verbascum densiflorum & - & 63.7 & - & - & - & - & - & - & - & - & - & - & - & - \\
\hline Pulsatilla pratensis & - & 59.8 & - & - & - & - & - & - & - & - & - & - & - & - \\
\hline Centaurea sumensis & - & 56.3 & - & - & - & - & - & - & - & - & - & - & - & - \\
\hline Jurinea ewersmanii & - & 52.6 & - & - & - & - & - & - & - & - & - & - & - & - \\
\hline Artemisia vulgaris & - & 52.4 & - & - & - & - & - & - & - & - & - & - & - & - \\
\hline Poa angustifolia & - & 50.8 & - & - & - & - & - & 19.3 & - & - & - & - & - & - \\
\hline Arabis glabra & - & 44.3 & - & - & - & - & - & - & - & - & - & - & - & - \\
\hline Veronica spicata s. incana & - & 43.6 & - & - & - & - & - & - & - & - & - & - & - & - \\
\hline Stellaria graminea & - & 39.6 & - & - & - & - & - & - & - & - & - & - & - & - \\
\hline Viola tricolor s. matutina & - & 37.7 & - & - & - & - & - & - & - & - & - & - & - & - \\
\hline Jurinea cyanoides s. tenuiloba & - & 34.2 & - & - & - & - & - & - & - & - & - & - & - & - \\
\hline Silene nutans & - & 33.6 & - & - & - & - & - & - & - & - & - & - & - & - \\
\hline Sedum telephium s. maximum & - & 32.5 & - & - & - & - & - & - & - & - & - & - & - & - \\
\hline Vincetoxicum hirundinaria & - & 30.9 & - & - & - & - & - & - & - & - & - & - & - & - \\
\hline Thesium ebracteatum & - & 27.9 & - & - & - & - & - & - & - & - & - & - & - & - \\
\hline Luzula pallescens & - & 27.9 & - & - & - & - & - & - & - & - & - & - & - & - \\
\hline Filipendula vulgaris & - & 27.9 & - & - & - & - & - & - & - & - & - & - & - & - \\
\hline Stachys recta & - & 27.9 & - & - & - & - & - & - & - & - & - & - & - & - \\
\hline Centaurea arenaria s. majorovii & - & 27.9 & - & - & - & - & - & - & - & - & - & - & - & - \\
\hline Artemisia austriaca & - & 27.9 & - & - & - & - & - & - & - & - & - & - & - & - \\
\hline Phleum phleoides & - & 27.9 & - & - & - & - & - & - & - & - & - & - & - & - \\
\hline Euphorbia esula s. tommasiniana & - & 27.9 & - & - & - & - & - & - & - & - & - & - & - & - \\
\hline Scorzonera purpurea & - & 27.9 & - & - & - & - & - & - & - & - & - & - & - & - \\
\hline Sempervivum ruthenicum & - & 27.8 & - & - & - & - & - & - & - & - & - & - & - & - \\
\hline Dianthus deltoides & - & - & 96.0 & - & - & - & - & - & - & - & - & - & - & - \\
\hline
\end{tabular}




\begin{tabular}{|c|c|c|c|c|c|c|c|c|c|c|c|c|c|c|}
\hline No. of syntaxa & 1 & 2 & 3 & 4 & 5 & 6 & 7 & 8 & 9 & 10 & 11 & 12 & 13 & 14 \\
\hline Number of relevés & 14 & 24 & 7 & 7 & 38 & 6 & 8 & 44 & 12 & 9 & 25 & 29 & 15 & 83 \\
\hline Armeria maritima s. elongata & - & - & 83.6 & - & - & - & - & - & - & - & - & - & - & - \\
\hline Deschampsia cespitosa & - & - & 74.4 & - & - & - & - & - & - & - & - & - & - & - \\
\hline Poapratensis & - & - & 74.4 & - & - & - & - & - & - & - & - & - & - & - \\
\hline Dactylis glomerata & - & - & 63.1 & - & - & - & - & - & - & - & - & - & - & - \\
\hline Anthoxanthum odoratum & - & - & 62.1 & - & - & - & - & - & - & - & - & - & - & - \\
\hline Agrostis capillaris & - & - & 61.9 & - & - & - & - & - & - & - & - & - & - & 11.6 \\
\hline Elymus repens & - & - & 54.0 & - & - & - & - & 16.8 & - & - & - & - & - & - \\
\hline Medicago lupulina & - & - & 52.0 & - & - & - & - & - & - & - & - & - & - & - \\
\hline Thuidium abietinum & - & - & 52.0 & - & - & - & - & - & - & - & - & - & - & - \\
\hline Cerastium arvense & - & - & 52.0 & - & - & - & - & - & - & - & - & - & - & - \\
\hline Lolium perenne & - & - & 52.0 & - & - & - & - & - & - & - & - & - & - & - \\
\hline Equisetum arvense & - & - & 48.6 & - & - & - & - & - & - & - & - & - & - & - \\
\hline Veronica chamaedrys & - & - & 40.8 & - & 11.9 & - & - & - & - & - & - & - & - & - \\
\hline Knautia arvensis & - & - & 40.6 & - & - & - & - & - & - & - & - & - & - & - \\
\hline Rumex thyrsiflorus & - & - & 39.5 & - & - & - & - & 19.7 & - & - & - & - & - & - \\
\hline Ranunculus acris & - & - & 36.6 & - & - & - & - & - & - & - & - & - & - & - \\
\hline Alyssum alyssoides & - & - & 36.6 & - & - & - & - & - & - & - & - & - & - & - \\
\hline Vicia hirsuta & - & - & 36.6 & - & - & - & - & - & - & - & - & - & - & - \\
\hline Festuca pratensis & - & - & 36.6 & - & - & - & - & - & - & - & - & - & - & - \\
\hline Lotus corniculatus & - & - & 36.6 & - & - & - & - & - & - & - & - & - & - & - \\
\hline Fragaria viridis & - & - & 36.6 & - & - & - & - & - & - & - & - & - & - & - \\
\hline Senecio sylvaticus & - & - & 36.6 & - & - & - & - & - & - & - & - & - & - & - \\
\hline Briza media & - & - & 36.6 & - & - & - & - & - & - & - & - & - & - & - \\
\hline Bromus commutatus & - & - & 36.6 & - & - & - & - & - & - & - & - & - & - & - \\
\hline Scirpus sylvaticus & - & - & 36.6 & - & - & - & - & - & - & - & - & - & - & - \\
\hline Alchemilla vallesiaca & - & - & 36.6 & - & - & - & - & - & - & - & - & - & - & - \\
\hline Plantago major & - & - & 36.6 & - & - & - & - & - & - & - & - & - & - & - \\
\hline Trisetum sibiricum & - & - & 36.6 & - & - & - & - & - & - & - & - & - & - & - \\
\hline Potentilla reptans & - & - & 36.6 & - & - & - & - & - & - & - & - & - & - & - \\
\hline Prunella vulgaris & - & - & 36.6 & - & - & - & - & - & - & - & - & - & - & - \\
\hline Leucanthemum vulgare & - & - & 36.6 & - & - & - & - & - & - & - & - & - & - & - \\
\hline Rubus caesius & - & - & 36.6 & - & - & - & - & - & - & - & - & - & - & - \\
\hline Centaurea jacea & - & - & 36.6 & - & - & - & - & - & - & - & - & - & - & - \\
\hline Plantago media & - & - & 36.6 & - & - & - & - & - & - & - & - & - & - & - \\
\hline Hylocomium splendens & - & - & 36.6 & - & - & - & - & - & - & - & - & - & - & - \\
\hline Agrimonia eupatoria & - & - & 36.6 & - & - & - & - & - & - & - & - & - & - & - \\
\hline Agrimonia procera & - & - & 36.6 & - & - & - & - & - & - & - & - & - & - & - \\
\hline Роа аппиа & - & - & 36.6 & - & - & - & - & - & - & - & - & - & - & - \\
\hline Daucus carota & - & - & 33.2 & - & - & - & - & - & - & - & - & - & - & - \\
\hline Clinopodium vulgare & - & - & 25.0 & - & - & - & 21.4 & - & - & - & - & - & - & - \\
\hline Thymus pulegioides & - & - & - & 53.1 & - & - & - & - & - & - & - & - & - & - \\
\hline Silene armeria & - & - & - & 52.0 & - & - & - & - & - & - & - & - & - & - \\
\hline Galeopsis ladanum & - & - & - & 52.0 & - & - & - & - & - & - & - & - & - & - \\
\hline Anthemis cotula & - & - & - & 49.7 & - & - & - & - & - & - & - & - & - & - \\
\hline Silene vulgaris & - & - & - & 44.3 & - & - & - & - & - & - & - & - & - & - \\
\hline Sedum telephium s. ruprechtii & 16.2 & - & - & 37.7 & - & - & - & - & - & - & - & - & - & - \\
\hline Trifolium alpestre & - & - & - & 36.6 & - & - & - & - & - & - & - & - & - & - \\
\hline Ononis arvensis & - & - & - & 36.6 & - & - & - & - & - & - & - & - & - & - \\
\hline Potentilla collina & - & - & - & 36.6 & - & - & - & - & - & - & - & - & - & - \\
\hline Dianthus arenarius s. pseudoserotinus & - & - & - & 36.6 & - & - & - & - & - & - & - & - & - & - \\
\hline Jovibarba globifera s. globifera & - & - & - & 36.6 & - & - & - & - & - & - & - & - & - & - \\
\hline Polypodium vulgare & - & - & - & 36.6 & - & - & - & - & - & - & - & - & - & - \\
\hline Centaurea rhenana s. pseudomaculosa & - & - & - & 33.6 & - & - & - & - & - & - & - & - & - & - \\
\hline Potentilla cinerea & - & 23.4 & - & 32.6 & - & - & - & - & - & - & - & 17.8 & - & - \\
\hline Chondrilla juncea & - & 15.9 & - & 30.6 & 11.4 & - & - & - & - & - & 20.7 & 18.8 & - & - \\
\hline Centaurea phrygia & - & - & - & - & 63.5 & - & - & - & - & - & - & - & - & - \\
\hline Artemisia scoparia & - & - & - & - & 57.6 & - & - & - & - & - & - & - & - & - \\
\hline Jasione montana & - & - & - & - & 45.1 & - & - & - & - & - & - & - & - & 20.4 \\
\hline Pteridium aquilinum & - & - & - & - & 35.5 & - & - & - & - & - & - & - & - & - \\
\hline Achillea millefolium & - & - & - & - & 32.7 & - & - & - & - & - & - & - & - & 16.8 \\
\hline Anthericum ramosum & - & - & - & - & 30.0 & - & - & - & - & - & - & - & - & - \\
\hline Oenothera rubricaulis & - & - & - & - & 28.1 & - & - & - & - & - & - & - & - & - \\
\hline Allium oleraceum & - & - & - & - & 27.2 & - & - & - & - & - & - & - & - & - \\
\hline Ajuga reptans & - & - & - & - & 27.2 & - & - & - & - & - & - & - & - & - \\
\hline Scabiosa ochroleuca & - & - & - & - & - & 100.0 & - & - & - & - & - & - & - & - \\
\hline Centaurea rhenana & - & - & - & - & - & 98.1 & - & - & - & - & - & - & - & - \\
\hline Silene tatarica & - & - & - & - & - & 80.8 & - & 15.1 & - & - & - & - & - & - \\
\hline Verbascum lychnitis & - & 9.7 & - & - & - & 71.2 & - & - & - & - & - & - & - & - \\
\hline Medicago sativa s. falcata & - & - & - & - & - & 69.4 & - & - & - & - & - & - & - & - \\
\hline Euphorbia esula & - & - & - & - & - & 62.8 & - & 18.6 & - & - & - & - & - & - \\
\hline Koeleria macrantha & - & - & - & - & - & 56.3 & - & - & - & - & - & - & - & - \\
\hline Viola arvensis & - & - & - & - & - & 49.5 & - & - & - & - & - & - & - & 8.9 \\
\hline Silene latifolia s. alba & - & - & - & - & - & 46.9 & - & - & - & - & - & - & - & - \\
\hline Seneciojacobaea & - & 13.4 & - & - & - & 44.7 & - & - & - & - & - & - & - & - \\
\hline Leontodon hispidus & - & - & - & - & - & 43.7 & - & - & - & - & - & - & - & 21.2 \\
\hline Erigeron annuus & - & - & - & - & - & 43.1 & - & 4.5 & - & - & - & - & - & - \\
\hline Echium vulgare & - & - & - & - & - & 34.5 & 24.2 & - & - & - & - & - & - & - \\
\hline Genista tinctoria & - & 23.1 & - & - & - & 33.1 & - & - & - & - & - & - & - & - \\
\hline Poa compressa & - & - & - & - & - & - & 85.0 & - & - & - & - & - & - & - \\
\hline Potentilla argentea & - & - & - & - & 14.8 & - & 54.6 & 12.4 & - & - & - & - & - & - \\
\hline
\end{tabular}




\begin{tabular}{|c|c|c|c|c|c|c|c|c|c|c|c|c|c|c|}
\hline No. of syntaxa & 1 & 2 & 3 & 4 & 5 & 6 & 7 & 8 & 9 & 10 & 11 & 12 & 13 & 14 \\
\hline Number of relevés & 14 & 24 & 7 & 7 & 38 & 6 & 8 & 44 & 12 & 9 & 25 & 29 & 15 & 83 \\
\hline Verbascum nigrum & - & - & - & - & - & - & 48.6 & - & - & - & - & - & - & - \\
\hline Melampyrum nemorosum & - & - & - & - & - & - & 48.6 & - & - & - & - & - & - & - \\
\hline Oenothera biennis & 15.5 & - & - & - & - & - & 45.5 & - & - & - & - & - & - & - \\
\hline Quercus robur & - & - & - & - & - & - & 42.9 & - & - & - & - & - & - & 7.2 \\
\hline Calamagrostis epigejos & - & 16.2 & - & - & - & - & 40.3 & 13.3 & - & - & - & - & - & - \\
\hline Hieracium pilosella & - & - & 23.8 & - & - & - & 35.8 & - & - & - & - & - & - & 17.0 \\
\hline Solidago virgaurea & - & - & - & - & - & - & 34.9 & - & - & - & - & - & - & 18.1 \\
\hline Elytrigia intermedia & - & - & - & - & - & - & 34.2 & - & - & - & - & - & - & - \\
\hline Polygonatum odoratum & - & - & - & - & - & - & 34.2 & - & - & - & - & - & - & - \\
\hline Luzula pilosa & - & - & - & - & - & - & 34.2 & - & - & - & - & - & - & - \\
\hline Lepidium campestre & - & - & - & - & - & - & 32.5 & - & - & - & - & - & - & - \\
\hline Acinos arvensis & - & - & - & - & - & - & 30.6 & - & - & - & - & - & - & - \\
\hline Bromus inermis & - & - & - & - & - & - & - & 68.6 & - & - & - & - & - & - \\
\hline Tanacetum vulgare & - & - & - & - & - & - & - & 62.4 & - & - & - & - & - & - \\
\hline Veronica spicata & - & - & - & - & - & - & - & 51.6 & - & - & - & - & - & - \\
\hline Achillea cartilaginea & - & - & - & - & - & - & - & 48.6 & - & - & - & - & - & - \\
\hline Eryngium planum & - & - & - & - & - & - & - & 46.3 & - & - & - & - & - & - \\
\hline Gratiola officinalis & - & - & - & - & - & - & - & 46.3 & - & - & - & - & - & - \\
\hline Veronica praecox & - & - & - & - & - & - & - & 43.9 & - & - & - & - & - & - \\
\hline Atriplex prostrata & - & - & - & - & - & - & - & 38.7 & - & - & - & - & - & - \\
\hline Veronica verna & - & - & - & - & - & - & - & 36.9 & - & - & - & - & - & - \\
\hline Allium angulosum & - & - & - & - & - & - & - & 32.6 & - & - & - & - & - & - \\
\hline Scirpus holoschoenus & - & - & - & - & - & - & - & 29.1 & - & - & - & - & - & - \\
\hline Sedum argutum & - & - & - & - & - & - & - & 26.3 & - & - & - & - & - & - \\
\hline Bromus squarrosus & - & - & - & - & - & - & - & 26.1 & - & - & - & - & - & - \\
\hline Bromus hordeaceus & - & - & - & - & - & - & - & - & 97.8 & - & - & - & - & - \\
\hline Spergularia rubra & - & - & - & - & - & - & - & - & 97.5 & - & - & - & - & - \\
\hline Arenaria serpyllifolia v. stepicola & - & - & - & - & - & - & - & - & 91.0 & - & - & - & - & - \\
\hline Spergula arvensis & - & - & - & - & - & - & - & - & 90.5 & - & - & - & - & - \\
\hline Polygonum aviculare & - & - & - & - & - & - & - & - & 80.9 & - & - & - & - & - \\
\hline Buglossoides arvensis s. arvensis & - & - & - & - & - & - & - & - & 72.3 & - & - & - & - & - \\
\hline Carex praecox & - & - & - & - & - & - & - & - & 54.4 & - & - & - & - & - \\
\hline Vicia cracca & - & 14.4 & - & - & - & - & - & - & 46.9 & - & - & - & - & - \\
\hline Linaria vulgaris & - & 13.4 & - & - & - & - & - & - & 44.7 & - & - & - & - & - \\
\hline Thesium procumbens & - & 20.2 & - & - & - & - & - & - & 28.3 & - & - & - & - & - \\
\hline Taraxacum officinale & - & - & - & - & - & - & - & 12.6 & 28.0 & - & - & - & - & - \\
\hline Hieracium umbellatum & - & 6.5 & - & - & - & - & - & - & - & 84.3 & - & - & - & - \\
\hline Agrostis vinealis & - & - & - & - & - & - & - & 16.6 & - & 70.1 & - & - & - & - \\
\hline Crepis tectorum agg. & - & - & - & - & - & - & - & - & - & 52.7 & - & - & - & - \\
\hline Herniaria polygama & - & - & - & - & - & - & - & - & 24.2 & 44.7 & 22.7 & 12.8 & - & - \\
\hline Linaria genistifolia & - & 12.6 & - & - & - & - & - & - & - & 29.9 & 18.8 & 18.0 & - & - \\
\hline Thymus serpyllum s. serpyllum & - & - & - & - & - & - & - & - & - & - & 93.4 & - & - & - \\
\hline Dianthus arenarius s. pseudosquarrosus & - & - & - & - & - & - & - & - & - & - & 69.5 & 21.4 & - & - \\
\hline $\begin{array}{l}\text { Jurinea cyanoides } \\
\text { Such }\end{array}$ & - & - & - & - & - & - & - & - & - & - & 69.4 & 21.6 & - & - \\
\hline Sedum telephium s. telephium & - & - & - & - & - & - & - & - & - & - & 56.8 & - & - & - \\
\hline Androsace septentrionalis & - & - & - & - & - & - & - & - & - & - & 47.6 & - & - & - \\
\hline Hierochloe stepporum & - & - & - & - & - & - & - & - & - & - & 43.4 & - & - & - \\
\hline Astragalus arenarius & - & - & - & - & - & - & - & - & - & - & 43.4 & - & - & - \\
\hline Sempervivum zeleborii & - & - & - & - & - & - & - & - & - & - & 27.3 & - & - & - \\
\hline Thymus pallasianus & - & - & - & - & - & - & - & - & - & - & - & 91.9 & - & - \\
\hline Minuartia viscosa & - & - & - & - & - & - & - & - & - & - & - & 84.2 & - & - \\
\hline Agropyron cristatum s. sabulosum & - & - & - & - & - & - & - & - & - & - & - & 75.4 & - & - \\
\hline Achillea micrantha & - & - & - & - & - & - & - & - & - & - & - & 75.4 & - & - \\
\hline Polygonum arenarium s. arenarium & - & - & - & - & - & - & - & - & - & - & - & 75.4 & - & - \\
\hline Alyssum tortuosum & - & - & - & - & - & - & - & - & - & - & - & 75.4 & - & - \\
\hline Astragalus varius & - & - & - & - & - & - & - & - & - & - & - & 75.4 & - & - \\
\hline Tragopogon brevirostris s. brevirostris & - & - & - & - & - & - & - & - & - & - & - & 73.0 & - & - \\
\hline Syrenia cana & - & - & - & - & - & - & - & - & - & - & - & 72.7 & - & - \\
\hline Dianthus platyodon & - & - & - & - & - & - & - & - & - & - & - & 70.6 & - & - \\
\hline Cladonia subcariosa & - & - & - & - & - & - & - & - & - & - & - & 70.6 & - & - \\
\hline Asperula graveolens & - & - & - & - & - & - & - & - & - & - & - & 70.6 & - & - \\
\hline Senecio borysthenicus & - & - & - & - & - & - & - & - & - & - & - & 69.9 & - & - \\
\hline Xanthopaemelia somloensis & - & - & - & - & - & - & - & - & - & - & - & 68.1 & - & - \\
\hline Anchusa gmelinii & - & - & - & - & - & - & - & - & - & - & - & 68.1 & - & - \\
\hline Dianthus squarrosus & - & - & - & - & - & - & - & - & - & - & - & 65.6 & - & - \\
\hline Stipa borysthenica & - & - & - & - & - & - & - & - & - & - & - & 64.9 & - & - \\
\hline Agropyron dasyanthum & - & - & - & - & - & - & - & - & - & - & - & 62.9 & - & - \\
\hline Asperula leiograveolens & - & - & - & - & - & - & - & - & - & - & - & 57.3 & - & - \\
\hline Scabiosa argentea & - & - & - & - & - & - & - & - & - & - & - & 57.3 & - & - \\
\hline Syrenia montana & - & - & - & - & - & - & - & - & - & - & - & 57.3 & - & - \\
\hline Seseli arenarium & - & - & - & - & - & - & - & - & - & - & - & 54.3 & - & - \\
\hline Minuartia setacea & - & - & - & - & - & - & - & - & - & - & - & 51.1 & - & - \\
\hline Allium guttatum & - & - & - & - & - & - & - & - & - & - & - & 47.8 & - & - \\
\hline Gypsophila paniculata & - & - & - & - & - & - & - & - & - & - & 18.3 & 44.8 & - & - \\
\hline Scorzonera ensifolia & - & - & - & - & - & - & - & - & - & - & - & 44.2 & - & - \\
\hline Pulsatilla patens & - & - & - & - & - & - & - & - & - & - & - & 43.7 & - & - \\
\hline Jurinea paczoskiana & - & - & - & - & - & - & - & - & - & - & - & 40.3 & - & - \\
\hline Carex ligerica & - & - & - & - & - & - & - & 12.9 & - & - & 16.1 & 39.0 & - & - \\
\hline Jurinea polyclonos & - & - & - & - & - & - & - & - & - & - & - & 36.0 & - & - \\
\hline Artemisia tschernieviana & - & - & - & - & - & - & - & - & - & - & - & 31.1 & - & - \\
\hline
\end{tabular}




\begin{tabular}{|c|c|c|c|c|c|c|c|c|c|c|c|c|c|c|}
\hline No. of syntaxa & 1 & 2 & 3 & 4 & 5 & 6 & 7 & 8 & 9 & 10 & 11 & 12 & 13 & 14 \\
\hline Number of relevés & 14 & 24 & 7 & 7 & 38 & 6 & 8 & 44 & 12 & 9 & 25 & 29 & 15 & 83 \\
\hline Diploschistes scruposus & - & - & - & - & - & - & - & - & - & - & - & 31.1 & - & - \\
\hline Cladonia pocillum & - & - & - & - & - & - & - & - & - & - & 17.9 & - & 63.4 & - \\
\hline Cladonia degenerans & - & - & - & - & - & - & - & - & - & - & - & - & 50.2 & - \\
\hline Cladonia mitis & - & - & - & - & - & - & - & - & - & - & - & - & - & 38.4 \\
\hline Hypochoeris radicata & - & - & - & - & - & - & - & - & - & - & - & - & - & 30.0 \\
\hline Cladonia rangiferina & - & - & - & - & - & - & - & - & - & - & - & - & - & 28.1 \\
\hline Scleranthus perennis & - & - & - & - & - & - & - & - & - & - & - & - & - & 26.5 \\
\hline Arenaria serpyllifolia & - & - & - & - & - & - & - & - & - & - & - & - & - & 26.1 \\
\hline Dicranum scoparium & - & - & - & - & - & - & - & - & - & - & - & - & - & 26.0 \\
\hline Logfia minima & - & - & - & - & - & - & - & - & - & - & - & - & - & 26.0 \\
\hline Secale sylvestre & 55.0 & - & - & - & - & - & - & - & 42.0 & - & - & 7.4 & - & - \\
\hline Anthemis ruthenica & 40.9 & - & - & - & - & - & - & 32.7 & - & - & - & - & - & - \\
\hline Lepidium densiflorum & 31.3 & - & - & - & - & - & - & - & 77.2 & - & - & - & - & - \\
\hline Asparagus officinalis & 27.4 & - & - & - & - & - & - & 34.5 & - & - & 16.5 & - & - & - \\
\hline Chamaecytisus ruthenicus & - & 47.9 & - & - & - & - & - & - & 36.6 & - & - & - & - & - \\
\hline Centaurea arenaria s. borysthenica & - & 45.1 & - & - & - & - & - & - & - & - & - & 61.6 & - & - \\
\hline Galium verum & - & 38.9 & - & - & 12.2 & - & - & 8.8 & - & 44.0 & - & - & - & - \\
\hline Rumexacetosa & - & 25.4 & - & - & - & 30.1 & 20.8 & - & - & - & - & - & - & - \\
\hline Euphorbia cyparissias & - & - & 29.2 & - & 29.7 & - & - & - & - & - & - & - & - & - \\
\hline Pinus sylvestris & - & - & - & 41.7 & - & 30.8 & - & - & - & - & - & - & - & 3.8 \\
\hline Festuca ovina & - & - & 24.2 & 33.8 & 49.6 & - & - & - & - & - & - & - & - & - \\
\hline Trifolium arvense & - & - & - & - & 32.0 & - & - & 31.4 & - & - & - & - & - & - \\
\hline Sedum sexangulare & - & - & - & - & - & 52.5 & - & 50.9 & - & - & - & - & - & - \\
\hline Berteroa incana & - & - & - & - & 8.7 & 47.1 & 23.3 & - & 26.0 & - & - & - & - & - \\
\hline Carexhirta & - & - & - & - & - & 45.6 & 42.1 & - & - & - & - & - & - & 7.8 \\
\hline Plantago lanceolata & - & - & - & - & - & 33.5 & - & 8.9 & 15.9 & 25.7 & - & - & - & - \\
\hline Artemisia absinthium & - & - & - & - & - & 30.6 & 35.4 & 10.8 & - & - & - & - & - & - \\
\hline Pinus sylvestris & - & - & - & - & - & 28.9 & 47.2 & - & - & - & - & - & - & 7.0 \\
\hline Genista tinctoria & - & - & - & - & - & 28.6 & 60.2 & - & - & - & - & - & - & - \\
\hline Sedum telephium & - & - & - & - & - & - & - & 31.9 & - & 49.4 & - & - & - & - \\
\hline Scleranthus annuus & - & - & - & - & - & - & - & - & 48.2 & 50.4 & - & - & - & - \\
\hline Sedum acres. acre & - & - & - & - & - & - & - & - & - & 70.7 & 28.3 & - & - & - \\
\hline Gypsophila muralis & - & - & - & - & - & - & - & - & - & 62.7 & 13.0 & 25.9 & - & - \\
\hline Rumex acetosella s. acetosella v. tenuifolius & - & - & - & - & - & - & - & - & - & 47.1 & 24.5 & 35.2 & 13.3 & - \\
\hline Silene conica & - & - & - & - & - & - & - & - & - & 46.3 & 15.2 & 32.3 & - & - \\
\hline Polytrichum piliferum & - & - & - & - & - & - & - & - & - & 40.5 & 35.8 & - & 20.7 & 11.9 \\
\hline Tortula ruralis & - & - & - & - & - & - & - & - & - & - & 58.8 & 54.1 & - & - \\
\hline Coelocaulon aculeatum & - & - & - & - & - & - & - & - & - & - & 56.1 & 50.4 & - & - \\
\hline Euphorbia seguierana & - & - & - & - & - & - & - & - & - & - & 49.3 & 61.1 & - & - \\
\hline Alyssum minutum & - & - & - & - & - & - & - & - & - & - & 49.3 & 48.4 & - & - \\
\hline Hieracium echioides & - & - & - & - & - & - & - & - & - & - & 37.0 & 43.5 & - & - \\
\hline Silene borysthenica & - & - & - & - & - & - & - & - & - & - & 36.9 & 43.3 & - & - \\
\hline Cerastium semidecandrum & - & 19.6 & - & - & - & - & - & - & - & - & 36.5 & 45.5 & - & - \\
\hline Racomitrium canescens & - & - & - & - & - & - & - & - & - & - & 29.1 & - & 35.8 & - \\
\hline Helichrysum arenarium & - & - & - & - & - & - & - & - & - & - & 25.9 & 40.1 & - & - \\
\hline Corynephorus canescens & - & - & - & - & - & - & - & - & - & - & 23.0 & - & 48.8 & 48.0 \\
\hline Festuca beckeri & 19.3 & 35.8 & - & - & - & - & - & - & - & - & 46.2 & 46.2 & - & - \\
\hline Achillea collina & - & 6.4 & 31.3 & - & - & 49.9 & 25.4 & - & - & - & - & - & - & - \\
\hline Thymus serpyllum & - & - & - & 54.2 & - & 31.4 & 28.5 & - & - & - & - & - & - & 4.7 \\
\hline Sedum acre & - & - & - & 32.2 & 7.7 & - & 25.8 & 28.5 & 15.8 & - & - & - & - & - \\
\hline Dianthus borbasii & - & - & - & - & - & - & 34.0 & 21.1 & - & 34.0 & 29.5 & - & - & - \\
\hline Erophila verna & - & - & - & - & - & - & - & - & - & 45.4 & 40.4 & 45.4 & 12.2 & - \\
\hline Ceratodon purpureus & - & - & - & - & - & - & 19.3 & - & - & 41.9 & 41.9 & 31.5 & 21.8 & - \\
\hline Myosotis stricta & - & - & - & - & - & - & - & - & - & 33.8 & 48.1 & 45.9 & 13.9 & - \\
\hline Bassia laniflora & - & - & - & - & - & - & - & - & - & 32.9 & 45.0 & 56.2 & - & - \\
\hline Cladina arbuscula & - & - & - & - & - & - & - & - & - & 29.2 & 44.5 & 36.7 & - & - \\
\hline Tragopogon brevirostris s. podolicus & - & - & - & - & - & - & - & - & - & 27.3 & 50.8 & 49.5 & - & - \\
\hline Cladonia rangiformis & - & - & - & - & - & - & - & - & - & - & 48.3 & 51.9 & 33.3 & - \\
\hline Artemisia campestris agg. & - & - & - & - & - & 25.3 & 25.3 & 24.0 & - & 25.3 & 23.1 & 25.3 & - & - \\
\hline Poabulbosa & - & - & - & - & - & - & - & - & 37.9 & 43.0 & 28.4 & 38.8 & - & - \\
\hline Koeleria glauca & - & - & - & - & 9.7 & - & - & - & 30.2 & 30.2 & 30.2 & 30.2 & - & - \\
\hline Cladonia foliacea & - & - & - & - & - & - & - & - & - & 41.5 & 40.9 & 48.6 & 27.2 & - \\
\hline Veronica dillenii & 12.6 & - & - & - & - & - & - & - & 32.6 & 32.6 & 28.1 & 30.7 & 32.6 & - \\
\hline Conyza canadensis & - & - & - & - & - & - & - & - & - & - & - & - & - & 23.8 \\
\hline Cladonia coniocraea & - & - & - & - & - & - & - & - & - & - & - & - & - & 23.7 \\
\hline Digitaria sanguinalis & - & - & - & - & - & - & - & - & - & - & - & - & - & 21.2 \\
\hline Polytrichum juniperinum & - & - & - & - & - & - & - & - & - & - & - & - & - & 21.2 \\
\hline Nardus stricta & - & - & - & - & - & - & - & - & - & - & - & - & - & 21.2 \\
\hline Campanula patula s. abietina & - & - & - & - & - & - & - & - & - & - & - & - & - & 21.2 \\
\hline Verbascum phlomoides & - & - & - & - & - & - & - & - & - & - & - & - & - & 18.3 \\
\hline Calluna vulgaris & - & - & - & - & - & - & - & - & - & - & - & - & - & 18.3 \\
\hline Cladonia mediterranea & - & - & - & - & - & - & - & - & - & - & - & - & - & 18.3 \\
\hline Setaria viridis & - & - & - & - & - & - & - & - & - & - & - & - & - & 18.3 \\
\hline Cladonia uncialis & - & - & - & - & - & - & - & - & - & - & - & - & - & 18.3 \\
\hline Agrostis canina & - & - & - & - & - & - & - & - & - & - & - & - & - & 17.0 \\
\hline Cladonia species & - & - & - & - & - & - & - & - & - & - & - & - & - & 13.7 \\
\hline Logfia arvensis & - & - & - & - & - & - & - & - & - & - & - & - & - & 12.8 \\
\hline Herniaria glabra & - & - & - & - & - & - & - & - & - & - & - & - & - & 10.6 \\
\hline Erigeron acer & - & - & - & - & - & - & - & - & - & - & - & - & - & 9.0 \\
\hline Brachythecium albicans & - & - & - & - & - & - & - & - & - & - & - & - & - & 8.3 \\
\hline
\end{tabular}




\begin{tabular}{|c|c|c|c|c|c|c|c|c|c|c|c|c|c|c|}
\hline No. of syntaxa & 1 & 2 & 3 & 4 & 5 & 6 & 7 & 8 & 9 & 10 & 11 & 12 & 13 & 14 \\
\hline Number of relevés & 14 & 24 & 7 & 7 & 38 & 6 & 8 & 44 & 12 & 9 & 25 & 29 & 15 & 83 \\
\hline Festuca rubra & - & - & - & - & - & - & - & - & - & - & - & - & - & 8.3 \\
\hline Hypericum perforatum & - & - & - & - & - & - & - & - & - & - & - & - & - & 5.6 \\
\hline Bromus tectorum & 24.8 & - & - & - & - & - & - & - & - & - & - & - & - & - \\
\hline Bromus secalimus & - & - & - & - & - & - & - & 20.6 & - & - & - & - & - & - \\
\hline Calamagrostis arundinacea & - & - & - & - & - & - & - & 20.6 & - & - & - & - & - & - \\
\hline Draba nemorosa & - & - & - & - & - & - & - & 20.6 & - & - & - & - & - & - \\
\hline Fallopia convolvulus & - & - & - & - & - & - & - & 20.6 & - & - & - & - & - & - \\
\hline Rhinanthus minor & - & - & - & - & - & - & - & 20.6 & - & - & - & - & - & - \\
\hline Lychnis viscaria & - & 15.1 & - & - & - & - & - & - & - & - & - & - & - & - \\
\hline Carex pediformis s. rhizodes & - & - & - & - & 22.1 & - & - & - & - & - & - & - & - & - \\
\hline Carex ericetorum & - & - & - & - & 17.9 & - & - & - & - & - & - & - & - & - \\
\hline Pinus sylvestris & - & 22.6 & - & - & - & - & - & - & - & - & - & - & - & - \\
\hline Juncus compressus & - & - & - & - & 22.1 & - & - & - & - & - & - & - & - & - \\
\hline Campanula patula & - & - & - & - & 18.4 & - & - & - & - & - & - & - & - & - \\
\hline Chenopodium rubrum & - & - & - & - & 22.1 & - & - & - & - & - & - & - & - & - \\
\hline Glechoma hederacea & - & - & - & - & 22.1 & - & - & - & - & - & - & - & - & - \\
\hline Dichanthium ischaemum & - & - & - & - & 22.1 & - & - & - & - & - & - & - & - & - \\
\hline Cerastium fontamum s. vulgare & - & - & - & - & 22.1 & - & - & - & - & - & - & - & - & - \\
\hline Teucrium chamaedrys & - & - & - & - & 21.1 & - & - & - & - & - & - & - & - & - \\
\hline Spergula morisonii & - & - & - & - & - & - & - & - & - & - & - & - & 24.9 & - \\
\hline Leontodon autumnalis & - & - & - & - & 9.1 & - & - & - & - & - & - & - & - & - \\
\hline Festuca valesiaca & - & - & - & - & - & - & - & 17.9 & - & - & - & - & - & - \\
\hline Descurainia sophia & - & - & - & - & 22.1 & - & - & - & - & - & - & - & - & - \\
\hline Fragaria vesca & - & - & 24.0 & 24.0 & - & - & - & - & - & - & - & - & - & - \\
\hline
\end{tabular}

Notes: numbers mark syntaxa: 1 - Veronico dillenii-Secaletum sylvestris; 2 -Chamaecytiso ruthenicae-Festucetum beckeri; 3 - Diantho deltoidis-Armerietum elongatae; 4 - Jasiono montanae-Thymetum serpylli; 5 - Jasiono montanae-Festucetum ovinae; 6 - Corynephoro-Silenetum tataricae, 7 - Artemisio campestris-Dianthetum borbasii; 8 - Artemisio dniproicae-Sedetum sexangularis; 9 - Veronico dillenii-Secaletum sylvestris (sensu Kovalenko); 10 - Diantho borbasii-Agrostietum syreistschikovii; 11 - Thymo angustifoliiFestucetum beckeri; 12-Centaureo borysthenicae-Festucetum beckeri; 13-Veronico dillenii-Corynephoretum; 14-Corniculario aculeatae-Corynephoretum canescentis.

The alliance Corynephorion canescentis combines coenoses in the structure of which mosses and especially lichens play a significant role, and which develop on weakly mobile sandy soils on fluvioglacial deposits, mainly in the Ukrainian Polissia. The Koelerion glaucae alliance includes multi-species communities growing on neutral sandy soils which developed mainly on alluvial deposits. They are common in the Ukrainian Polissia and, less often, in the forest-steppe on the pine-forest terraces of rivers and natural levees. Their territorial differentiation is determined by the relief and soil reaction.

According to the results of ordination analysis of communities in the class Koelerio-Corynephoretea canescentis, it was found that temperature regime and climate continentality play a leading role in their ecological differentiation (Fig. 4). These parameters are most important for coenoses within Corynephorion canescentis. Also, the distribution of communities is significantly affected by the gradients of ombroregime and soil humidity. Communities of Corynephoro-Silenetum tataricae, Thymo angustifolii-Festucetum beckeri, Jasiono montanae-Thymetum serpylli and Jasiono montanaeFestucetum ovinae associations are more "sensitive" to changes in these parameters. Communities of the last two syntaxa will also respond to concentration of available nitrogen forms in soil substrate. Variability of damping (fH) can be considered the leading ecological factor for the coenoses Diantho borbasii-Agrostietum syreistschikovii, Veronico dillenii-Secaletum sylvestris and Chamaecytiso ruthenicae-Festucetun beckeri. Communities of the Diantho deltoidis-Armerietum elongatae association were described on the territory of the Ukrainian Roztochia on specific moraine deposits, and their distribution in the ecological space determines content of carbonates and other salts in the soil. Diantho deltoidis-Armerietum elongatae, Corynephoro-Silenetum tataricae, Artemisio dniproicae-Sedetum sexangularis and Thymo angustifoliiFestucetum beckeri communities differ significantly in their ecological amplitude (Fig. 4).

Table 2

Synoptic table of the Festucetea vaginatae class

\begin{tabular}{|c|c|c|c|c|c|c|c|c|c|c|c|c|c|c|c|c|c|c|c|c|c|c|c|c|c|c|c|}
\hline No. of syntaxa & 1 & 2 & 3 & 4 & 5 & 6 & 7 & 8 & 9 & 10 & 11 & 12 & 13 & 14 & 15 & 16 & 17 & 18 & 19 & 20 & 21 & 22 & 23 & 24 & 25 & 26 & 27 \\
\hline Number of relevés & 10 & 17 & 20 & 13 & 18 & 4 & 9 & 43 & 9 & 10 & 14 & 5 & 10 & 7 & 5 & 12 & 10 & 13 & 21 & 8 & 47 & 16 & 10 & 10 & 10 & 92 & 47 \\
\hline Juncus bufonius & 51 & 21 & - & - & - & - & - & - & - & - & - & - & - & - & - & - & - & - & - & - & - & - & - & - & - & - & - \\
\hline Trifolium fragiferum & 44 & - & - & - & - & - & - & - & - & - & - & - & - & - & - & - & - & - & - & - & - & - & - & - & - & - & - \\
\hline Aster tripolium & 44 & - & - & - & - & - & - & - & - & - & - & - & - & - & - & - & - & - & - & - & - & - & - & - & - & - & - \\
\hline Sonchus palustris & 44 & - & - & - & - & - & - & - & - & - & - & - & - & - & - & - & - & - & - & - & - & - & - & - & - & - & - \\
\hline Carex extensa & 44 & - & - & - & - & - & - & - & - & - & - & - & - & - & - & - & - & - & - & - & - & - & - & - & - & - & - \\
\hline Barbarea stricta & 44 & - & - & - & - & - & - & - & - & - & - & - & - & - & - & - & - & - & - & - & - & - & - & - & - & - & - \\
\hline Odontites verna s. serotina & 41 & - & - & - & - & - & - & - & - & - & 18 & - & - & - & - & - & - & - & - & - & - & - & - & - & - & - & - \\
\hline Medicago lupulina & 41 & - & - & - & - & - & - & - & - & - & - & - & - & - & - & - & - & - & - & - & - & 15 & - & - & - & - & - \\
\hline Limonium bellidifolium & 38 & - & - & - & - & - & - & - & - & - & - & - & - & - & - & - & - & - & - & - & - & - & - & - & - & - & - \\
\hline Plantago major & 37 & - & - & - & - & - & - & - & - & - & - & - & - & - & - & - & - & - & - & - & - & - & - & - & - & - & - \\
\hline Agrostis gigantea s. maeotica & 34 & - & - & - & - & - & - & - & - & - & - & - & - & - & - & - & - & - & - & - & - & - & - & - & - & - & - \\
\hline Phragmites australis & 34 & 19 & - & - & 18 & - & - & - & - & - & - & - & - & - & - & - & - & - & - & - & - & - & - & - & - & - & - \\
\hline Rumex acetosa & 31 & - & - & - & - & - & - & - & - & - & - & - & - & - & - & - & - & - & - & - & - & - & - & - & - & - & - \\
\hline Inula britannica & 31 & - & - & - & - & - & - & - & - & - & - & - & - & - & - & - & - & - & - & - & - & - & - & - & - & - & - \\
\hline Elytrigia intermedia & 31 & - & - & - & - & - & - & - & - & - & - & - & - & - & - & - & - & - & - & - & - & - & - & - & - & - & - \\
\hline Aster sedifolius s. dracunculoides & 31 & - & - & - & - & - & - & - & - & - & - & - & - & - & - & - & - & - & - & - & - & - & - & - & - & - & - \\
\hline Xanthium strumarium & 29 & - & - & - & - & - & - & - & - & - & - & - & - & - & - & - & - & - & - & - & - & - & - & - & - & - & - \\
\hline Cirsium ukranicum & 28 & - & - & - & - & - & - & - & - & - & - & - & - & - & - & - & - & - & - & - & - & - & - & - & - & - & - \\
\hline Bromus squarrosus & 28 & 16 & - & - & 14 & - & - & - & - & - & 25 & - & - & - & - & - & - & - & - & - & - & 14 & - & - & - & - & - \\
\hline Chrysopogon gryllus & 22 & 85 & - & - & - & - & - & - & - & - & - & - & - & - & - & - & - & - & - & - & - & - & - & - & - & - & - \\
\hline Picris hieracioides s. hieracioides & - & 36 & - & - & - & - & - & - & - & - & - & - & - & - & - & - & - & - & - & - & - & - & - & - & - & - & - \\
\hline Asparagus litoralis & - & 35 & - & - & - & - & - & - & - & - & - & - & - & - & - & - & - & - & - & - & - & - & - & - & - & - & - \\
\hline Convolvulus lineatus & - & 34 & - & - & - & - & - & - & - & - & - & - & - & - & - & - & - & - & - & - & - & - & - & - & - & - & - \\
\hline Orchis morio s. picta & - & 34 & - & - & - & - & - & - & - & - & - & - & - & - & - & - & - & - & - & - & - & - & - & - & - & - & - \\
\hline Plantago maritima & 21 & 33 & - & - & - & - & - & - & - & - & - & - & - & - & - & - & - & - & - & - & - & - & - & - & - & - & - \\
\hline Schoenus nigricans & - & 32 & - & - & - & - & - & - & - & - & - & - & - & - & - & - & - & - & - & - & - & - & - & - & - & - & - \\
\hline Rumex acetosella & - & 31 & - & - & - & - & - & - & - & - & - & - & - & - & - & - & - & - & - & - & 18 & - & - & - & - & - & 12 \\
\hline
\end{tabular}




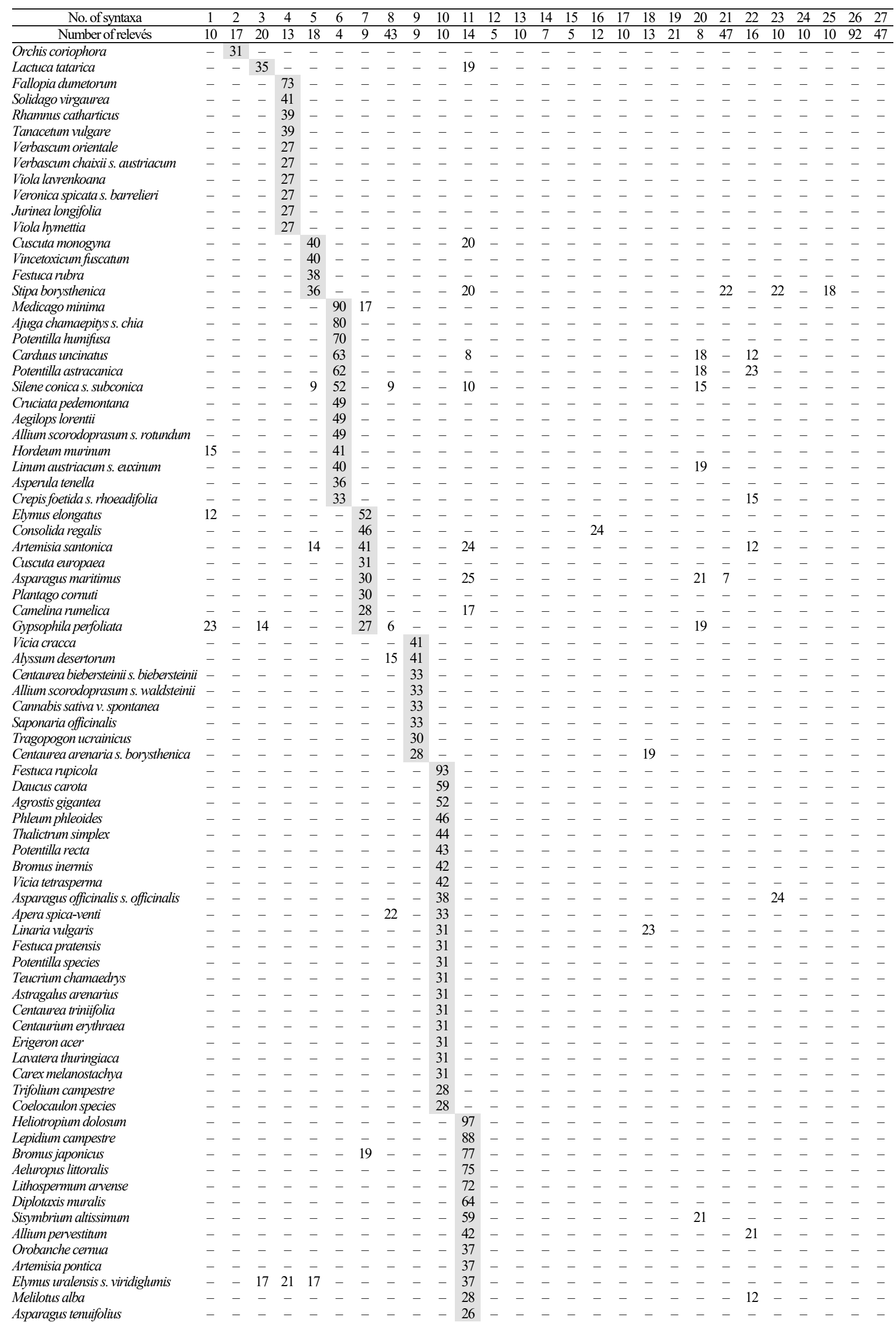




\begin{tabular}{|c|c|c|c|c|c|c|c|c|c|c|c|c|c|c|c|c|c|}
\hline No. of syntaxa & 1 & 2 & 3 & 4 & 5 & 6 & 7 & 8 & 9 & 10 & 11 & 12 & 13 & 14 & 15 & 16 & 17 \\
\hline Number of relevés & 10 & 17 & 20 & 13 & 18 & 4 & 9 & 43 & 9 & 10 & 14 & 5 & 10 & 7 & 5 & 12 & 10 \\
\hline Polygonum arenarium s. pulchellum & - & - & - & - & - & - & - & - & - & - & 26 & - & - & - & - & - & - \\
\hline Halimione verrucifera & - & - & - & - & - & - & - & - & - & - & 26 & - & - & - & - & - & - \\
\hline Thesium linophyllon & - & - & - & - & - & - & - & - & - & - & 26 & - & - & - & - & - & - \\
\hline Tragus racemosus & - & - & - & - & - & - & - & - & - & - & - & 72 & - & - & - & - & - \\
\hline Lotus tenuis & - & - & - & - & - & - & - & - & - & - & - & 66 & - & - & - & - & - \\
\hline Eragrostis pilosa & - & - & - & - & - & - & - & - & - & - & - & 48 & - & - & 22 & - & - \\
\hline Corispermum ucrainicum & - & - & - & - & 10 & - & 24 & - & - & - & - & 45 & - & - & - & - & - \\
\hline Thlaspi arvense & - & - & - & - & - & - & - & - & - & - & - & 34 & - & - & - & - & - \\
\hline Corispermum nitidum & - & - & - & - & - & - & - & - & - & - & - & - & 99 & - & - & - & - \\
\hline Linaria odora & - & - & - & - & - & - & - & - & - & - & - & - & 99 & - & - & - & - \\
\hline Tribulus terrestris & - & - & - & - & - & - & - & - & - & - & 24 & - & 77 & - & - & - & - \\
\hline Linaria dulcis & - & - & - & - & - & - & - & - & - & - & - & - & - & 93 & - & - & - \\
\hline Jurinea ewersmanii & - & - & - & - & - & - & - & - & - & - & - & - & - & 75 & - & - & - \\
\hline Digitaria sanguinalis & - & - & - & - & - & - & - & - & - & - & - & - & - & 72 & - & - & - \\
\hline Silene borysthenica & - & - & - & - & - & - & - & - & - & - & - & - & - & 60 & - & - & - \\
\hline Jurinea cyanoides s. tenuiloba & - & - & - & - & - & - & - & - & - & - & - & - & - & 59 & - & - & - \\
\hline Ambrosia artemisiifolia & - & - & - & - & - & - & - & - & - & - & - & - & - & 55 & - & - & - \\
\hline Lactuca serriola & - & - & - & 16 & - & - & - & - & - & - & - & - & - & 51 & - & - & - \\
\hline Calamagrostis epigejos & - & - & 7 & 24 & - & - & - & - & - & 14 & - & - & - & 38 & - & - & - \\
\hline Sisymbrium orientale & - & - & - & - & - & - & - & - & 16 & - & - & - & - & - & 69 & - & - \\
\hline Anchusa officinalis & - & - & - & - & - & - & - & - & - & - & - & - & - & - & 58 & - & - \\
\hline Achillea collina & - & - & - & - & - & - & - & - & - & - & - & - & - & - & 52 & - & - \\
\hline Sisymbrium polymorphum & - & 11 & 9 & - & - & - & - & - & - & 14 & - & - & - & - & 50 & - & - \\
\hline Inula salicina & - & 16 & - & - & - & - & - & - & - & - & - & - & - & - & 29 & - & - \\
\hline Ephedra distachya & - & - & - & - & 10 & - & - & - & - & - & 11 & - & - & - & - & 54 & - \\
\hline Dianthus capitatus & - & - & - & - & - & - & - & - & - & - & - & - & - & - & - & 28 & - \\
\hline Dianthus bessarabicus & - & - & - & - & - & - & - & - & - & - & - & - & - & - & - & 28 & - \\
\hline Onobrychis arenaria & - & - & - & - & - & - & - & - & - & - & - & - & - & - & - & 28 & - \\
\hline Marrubium pestalozzae & - & - & - & - & - & - & - & - & - & - & - & - & - & - & - & 28 & - \\
\hline Sinapis alba & - & - & - & - & - & - & - & - & - & - & - & - & - & - & - & 28 & - \\
\hline Echinops ritro & - & - & - & - & - & - & - & - & - & - & - & - & - & - & - & 28 & - \\
\hline Thymus dimorphus & - & - & 22 & - & 16 & - & - & - & - & - & 22 & - & - & - & - & 27 & - \\
\hline Achillea setacea & - & - & - & - & - & - & - & - & - & - & - & - & - & - & - & - & 89 \\
\hline Potentilla argentea & - & - & - & - & - & - & - & - & - & - & - & - & - & - & - & - & 78 \\
\hline Hieracium pilosella & - & - & - & - & - & - & - & - & - & - & - & - & - & - & - & - & 76 \\
\hline Poa angustifolia & - & - & - & - & - & - & - & - & - & 21 & - & - & - & - & - & - & 73 \\
\hline Trifolium arvense & - & - & - & - & - & - & - & - & - & - & - & - & - & - & - & - & 72 \\
\hline Cerastium fontanum s. vulgare & - & - & - & - & - & - & - & - & - & - & - & - & - & - & - & - & 70 \\
\hline Taraxacum officinale & - & - & - & - & - & - & - & - & - & - & - & - & - & - & - & - & 57 \\
\hline Artemisia austriaca & - & - & - & - & - & - & - & - & - & - & - & 21 & - & - & - & - & 56 \\
\hline $\begin{array}{l}\text { Jurinea consanguineas. } \\
\text { arachnoidea }\end{array}$ & - & - & - & - & - & - & - & - & - & - & - & - & - & - & - & - & 54 \\
\hline Sedum acre & - & - & - & - & - & - & - & - & - & - & - & - & - & - & - & - & 46 \\
\hline Роа аппиа & - & - & - & - & - & - & - & - & - & - & - & - & - & - & - & - & 44 \\
\hline Galium album & - & - & - & - & - & - & - & - & - & - & - & - & - & - & - & - & 44 \\
\hline Thesium arvense & - & - & - & - & - & - & - & - & - & - & 15 & - & - & - & - & - & 41 \\
\hline Stellaria graminea & - & - & - & - & - & - & - & - & - & - & - & - & - & - & - & - & 39 \\
\hline Herniaria glabra & - & - & - & - & - & - & - & - & - & - & - & - & - & - & - & - & 36 \\
\hline Sanguisorba officinalis & - & - & - & - & - & - & - & - & - & - & - & - & - & - & - & - & 31 \\
\hline Convolvulus arvensis & - & - & - & - & - & - & - & - & - & - & - & - & - & - & - & - & 28 \\
\hline Jurinea polyclonos & - & - & - & - & - & - & - & - & - & - & - & - & - & - & - & - & - \\
\hline Achillea nobilis & - & - & - & - & - & - & - & - & - & - & - & - & - & 22 & - & - & 14 \\
\hline Centaurea arenarias. & - & - & - & - & - & - & - & - & - & - & - & - & - & - & - & - & - \\
\hline Asparagus officinalis & - & - & - & - & - & - & - & - & - & - & - & - & - & - & - & - & - \\
\hline Jasione montana & - & - & - & - & - & - & - & - & - & - & - & - & - & - & - & - & - \\
\hline Tragopogon ruthenicus s. tanaiticus & - & - & - & - & - & - & - & - & - & - & - & - & - & - & - & - & - \\
\hline Agrostis vinealis & - & - & - & - & - & - & - & - & - & - & - & - & - & - & - & - & - \\
\hline Gypsophila paniculata & - & - & - & - & - & - & - & - & - & - & - & - & - & - & - & 14 & - \\
\hline Reseda lutea & - & - & - & - & - & - & - & - & - & - & - & - & - & - & - & - & - \\
\hline Verbascum thapsus & - & - & - & - & - & - & - & - & - & - & - & - & - & - & - & - & - \\
\hline Salix acutifolia & - & - & - & - & - & - & - & - & - & - & - & - & - & - & - & - & - \\
\hline Potentilla cinerea & - & - & - & - & - & - & - & - & - & - & - & - & - & - & - & - & - \\
\hline n robertian & - & - & - & - & - & - & - & - & - & - & - & - & - & - & - & - & - \\
\hline mellus & - & - & - & - & - & - & - & - & - & - & - & - & - & - & - & - & - \\
\hline Pulsatilla vulgaris & - & - & - & - & - & - & - & - & - & - & - & - & - & - & - & - & - \\
\hline Astragalus sulcatus & - & - & - & - & - & - & - & - & - & - & - & - & - & - & - & - & - \\
\hline Viola ambigua & - & - & - & - & - & - & - & - & - & - & - & - & - & - & - & - & - \\
\hline Potentilla thyrsiflora & - & - & - & - & - & - & - & - & - & - & - & - & - & - & - & - & - \\
\hline Silene chlorantha & - & - & - & - & - & - & - & - & - & - & - & - & - & - & - & - & - \\
\hline Equisetum arvense & - & - & - & - & - & - & - & - & - & - & - & - & - & - & - & - & - \\
\hline Elymus hispidus s. barbulatus & - & - & - & - & - & - & - & - & - & - & - & - & - & - & - & - & - \\
\hline Melissa officinalis & - & - & - & - & - & - & - & - & - & - & - & - & - & - & - & - & - \\
\hline npressa & - & - & - & - & - & - & - & - & - & - & - & - & - & - & - & - & - \\
\hline Delphinium cuneatum & - & - & - & - & - & - & - & - & - & - & - & - & - & - & - & - & - \\
\hline Melilotus officinalis & - & - & - & - & - & - & - & - & - & - & - & - & - & - & - & - & - \\
\hline Leymus racemosus s. sabulosus & - & - & - & - & - & - & - & - & - & - & - & - & - & - & - & - & - \\
\hline Elymus farctus s. bessarabicus & - & - & - & - & - & - & - & - & - & - & - & - & - & - & - & - & - \\
\hline Eryngium maritimum & - & - & - & - & - & - & - & - & - & - & - & - & - & - & - & - & - \\
\hline Astrodaucus littoralis & - & - & - & - & - & - & - & - & - & - & - & - & - & - & - & - & - \\
\hline Festuca arundinacea s. orientalis & - & - & 19 & - & - & - & - & - & - & - & - & - & - & - & - & - & - \\
\hline
\end{tabular}




\begin{tabular}{|c|c|c|c|c|c|c|c|c|c|c|c|c|c|c|c|c|c|}
\hline No. of syntaxa & 1 & 2 & 3 & 4 & 5 & 6 & 7 & 8 & 9 & 10 & 11 & 12 & 13 & 14 & 15 & 16 & 17 \\
\hline Number of relevés & 10 & 17 & 20 & 13 & 18 & 4 & 9 & 43 & 9 & 10 & 14 & 5 & 10 & 7 & 5 & 12 & 10 \\
\hline Stipa capillata & - & - & - & - & - & - & - & - & - & - & - & - & - & - & - & - & - \\
\hline Coronilla varia & - & - & - & - & - & - & - & - & - & - & - & - & - & - & - & - & - \\
\hline Dianthus pseudarmeria & - & - & - & - & 17 & - & - & - & - & - & 16 & - & - & - & - & - & - \\
\hline Allium flavum s. tauricum & - & - & - & - & 19 & - & - & - & - & - & 11 & - & - & - & - & - & - \\
\hline Trigonella monspeliaca & - & - & - & - & 17 & - & - & - & - & - & 14 & - & - & - & - & - & - \\
\hline Descurainia sophia & - & - & - & - & - & - & - & - & - & - & - & - & - & - & - & - & - \\
\hline Silene chersonensis & - & - & - & - & - & - & - & - & - & - & - & - & - & - & - & - & - \\
\hline Buglossoides arvensis & - & - & - & - & - & - & - & - & - & - & - & - & - & - & - & - & - \\
\hline Alcea rugosa & - & - & - & - & - & - & - & - & - & - & - & - & - & - & - & - & - \\
\hline Cladonia species & - & - & - & - & - & - & - & - & - & - & - & - & - & - & - & 21 & - \\
\hline Gagea arvensis & - & - & - & - & - & - & - & - & - & - & - & - & - & - & - & - & - \\
\hline Gagea dubia & - & - & - & - & - & - & - & - & - & - & - & - & - & - & - & - & - \\
\hline Saxifraga tridactylites & - & - & - & - & - & - & - & - & - & - & - & - & - & - & - & - & - \\
\hline Salvia aethiopis & - & - & - & - & - & - & - & - & - & - & - & - & - & - & - & - & - \\
\hline Agropyron cimmericum & - & - & - & - & - & - & - & - & - & - & - & - & - & - & - & - & - \\
\hline Cirsium alatum & - & - & - & - & - & - & - & - & - & - & - & - & - & - & - & - & - \\
\hline Cirsium vulgare & 20 & - & - & - & - & - & - & - & - & - & - & - & - & - & - & - & - \\
\hline Ceratodon purpureus & - & - & - & - & - & - & - & - & - & - & - & - & - & - & - & - & - \\
\hline Cladonia convoluta & - & - & - & - & - & - & - & - & - & - & - & - & - & - & - & - & - \\
\hline Allium paniculatum & - & - & - & - & - & - & - & - & - & - & - & - & - & - & - & - & - \\
\hline Neofuscelia species & - & - & - & - & - & - & - & - & - & - & - & - & - & - & - & - & - \\
\hline Scorzonera ensifolia & - & - & - & - & - & - & - & - & - & - & - & - & - & - & - & - & - \\
\hline Achillea micrantha & - & - & - & - & - & - & - & - & - & - & - & - & 18 & - & - & - & - \\
\hline Rumex confertus & - & - & - & - & - & - & - & - & - & - & - & - & - & - & - & - & - \\
\hline Crepis sancta & - & - & - & - & - & - & - & - & - & - & - & - & - & - & - & - & - \\
\hline Artemisia absinthium & - & - & - & - & - & - & - & - & - & - & - & - & - & - & - & - & - \\
\hline Papaver dubium & - & - & - & - & - & - & - & - & - & - & - & - & - & - & - & - & - \\
\hline Cichorium intybus & 23 & - & - & - & - & - & - & - & - & - & - & - & - & - & - & - & 23 \\
\hline Lepidium latifolium & - & - & - & - & - & - & - & - & - & - & - & - & - & - & - & - & - \\
\hline Koeleria macrantha & - & - & - & - & - & - & - & - & - & - & - & - & - & - & - & - & - \\
\hline Chenopodium opulifolium & - & - & - & - & - & - & - & - & - & - & - & - & - & - & - & - & - \\
\hline Falcaria vulgaris & - & - & - & - & - & - & - & - & - & - & - & - & - & - & - & - & 21 \\
\hline Lamium amplexicaule & - & - & - & - & - & - & - & - & - & - & - & - & - & - & - & - & - \\
\hline Echium vulgare & - & - & - & - & - & - & - & - & - & - & - & - & - & - & - & - & 15 \\
\hline Bassia prostrata & - & - & - & - & - & - & - & 8 & - & - & - & - & - & - & - & - & - \\
\hline Elymus farctus & - & - & - & - & - & - & - & - & - & - & - & - & - & - & - & - & - \\
\hline Artemisia tschernieviana & - & - & - & - & - & - & - & - & - & - & 24 & - & - & - & - & - & - \\
\hline Alyssum borzaeanum & - & - & - & - & - & - & - & - & - & - & - & - & - & - & - & - & - \\
\hline Asperula setulosa & - & - & - & - & 7 & - & - & - & - & - & 22 & - & - & - & - & - & - \\
\hline Silene exaltata & - & - & - & - & - & - & - & - & - & - & - & - & - & - & - & - & - \\
\hline Orobanche coerulescens & - & - & - & - & - & - & - & - & - & - & - & - & - & - & - & - & - \\
\hline Onosma arenaria & - & - & - & - & - & - & - & - & - & - & - & - & - & - & - & - & - \\
\hline Leymus racemosus & - & - & - & - & - & - & - & - & - & - & - & - & - & - & - & - & - \\
\hline Linaria genistifolia & - & - & - & - & - & - & - & - & - & - & - & - & - & - & - & - & - \\
\hline Inula salicina s. aspera & - & - & - & - & - & - & - & - & - & 21 & - & - & - & - & - & - & - \\
\hline $\begin{array}{l}\text { Rumex acetosella s. acetosella } v . \\
\text { temuifolius }\end{array}$ & - & - & - & - & - & - & - & - & - & - & - & - & - & - & - & - & - \\
\hline Vicia hirsuta & - & - & - & - & - & - & - & - & - & - & - & - & - & - & - & - & - \\
\hline Centaurium pulchellum & - & - & - & - & - & - & - & - & - & - & - & - & - & - & - & - & - \\
\hline Veronica spicata & - & - & - & - & - & - & - & - & - & - & - & - & - & - & - & - & - \\
\hline Verbascum phoeniceum & - & - & - & - & - & - & - & - & - & - & - & - & - & - & - & - & - \\
\hline Echinops ritro s. ruthenicus & - & - & - & - & - & - & - & - & - & - & - & - & - & - & - & - & - \\
\hline Scabiosa ochroleuca & - & - & - & - & - & - & - & - & - & - & - & - & - & - & - & - & - \\
\hline Thalictrum minus & - & - & - & - & - & - & - & - & - & - & - & - & - & - & - & - & - \\
\hline Euphorbia cyparissias & - & - & - & - & 21 & - & - & - & - & - & - & - & - & - & - & - & - \\
\hline Centaurea rhenana & - & - & - & - & - & - & - & - & - & - & - & - & - & - & - & - & - \\
\hline Chamaecytisus ruthenicus & - & - & - & - & - & - & - & - & - & - & - & - & - & - & - & - & - \\
\hline Apera spica-venti s. maritima & 58 & 9 & 10 & - & - & 42 & - & - & - & - & - & - & - & - & - & - & - \\
\hline Lotus corniculatus & 35 & - & - & - & - & - & - & - & - & - & - & - & - & - & - & - & 35 \\
\hline Matricaria perforata & 30 & - & - & - & - & - & - & - & - & - & - & - & - & - & - & - & - \\
\hline Milium vernale & 21 & 31 & - & 35 & - & - & - & - & - & - & - & - & - & - & - & - & - \\
\hline Scirpus holoschoenus & - & 14 & 32 & 25 & - & - & - & - & - & 14 & 16 & - & - & - & - & - & - \\
\hline Salix rosmarinifolia & - & - & - & 63 & - & - & - & - & - & - & - & - & - & - & - & - & - \\
\hline Galium ruthenicum & - & - & - & 41 & - & - & - & - & - & 38 & - & - & - & - & - & - & - \\
\hline Conyza canadensis & - & - & - & 29 & - & - & - & - & - & - & - & - & - & 47 & - & - & - \\
\hline Arabidopsis toxophylla & - & - & - & - & 38 & - & - & - & - & - & 29 & - & - & - & - & - & - \\
\hline Bassia sedoides & - & - & - & - & 32 & - & - & - & - & - & 33 & - & - & - & - & - & - \\
\hline Arenaria serpyllifolia & - & - & - & - & 26 & - & - & - & - & - & - & - & - & - & - & - & - \\
\hline Thlaspi perfoliatum & - & - & - & - & 26 & - & - & - & - & - & 49 & - & - & - & - & - & - \\
\hline Anthemis ruthenica & - & - & - & - & 7 & 40 & - & - & - & - & - & 56 & - & - & - & - & - \\
\hline Limonium meyeri & - & - & - & - & - & 34 & - & - & - & - & - & - & - & - & - & - & - \\
\hline Arenaria serpyllifolia s. leptoc & - & - & - & - & - & 30 & - & - & - & - & - & - & - & - & - & - & - \\
\hline Marrubium peregrinum & - & - & - & - & - & 30 & - & - & - & - & 61 & - & - & - & - & - & - \\
\hline Poa bulbosa & - & - & - & - & - & 29 & - & - & - & - & - & - & - & - & - & - & 20 \\
\hline Artemisia taurica & - & - & - & - & - & - & 56 & - & - & - & 27 & - & - & - & - & - & - \\
\hline Senecio vernalis & - & - & - & - & - & - & 34 & - & - & - & 50 & - & - & - & - & - & - \\
\hline Galium humifusum & - & - & - & - & - & - & 34 & - & - & - & 20 & - & - & - & - & - & - \\
\hline Xanthopaemelia camschadalis & - & - & 18 & - & 10 & - & 28 & - & - & - & 51 & - & - & - & - & 19 & - \\
\hline Anthemis arvensis & - & - & - & - & - & - & - & 30 & 32 & - & - & - & - & - & - & - & - \\
\hline Linum perenne & - & - & - & - & - & - & - & - & - & 45 & - & - & - & - & - & - & - \\
\hline
\end{tabular}




\begin{tabular}{|c|c|c|c|c|c|c|c|c|c|c|c|c|c|c|c|c|c|c|c|c|c|c|c|c|c|c|c|}
\hline No. of syntaxa & 1 & 2 & 3 & 4 & 5 & 6 & 7 & 8 & 9 & 10 & 11 & 12 & 13 & 14 & 15 & 16 & 17 & 18 & 19 & 20 & 21 & 22 & 23 & 24 & 25 & 26 & 27 \\
\hline Number of relevés & 10 & 17 & 20 & 13 & 18 & 4 & 9 & 43 & 9 & 10 & 14 & 5 & 10 & 7 & 5 & 12 & 10 & 13 & 21 & 8 & 47 & 16 & 10 & 10 & 10 & 92 & 47 \\
\hline Picris hieracioides & - & - & - & - & - & - & - & - & - & 25 & 49 & - & - & - & - & - & - & - & - & - & - & - & 18 & - & - & - & - \\
\hline Eragrostis minor & - & - & - & - & - & - & - & - & - & - & 63 & - & - & - & - & - & - & - & - & 27 & - & - & - & - & - & - & - \\
\hline Alyssum hirsutum & - & - & 13 & - & 15 & - & 24 & - & - & - & 38 & - & - & - & - & - & - & - & - & 36 & - & 18 & - & - & - & - & - \\
\hline Silene spergulifolia & - & - & - & - & - & - & - & - & - & - & 31 & - & - & - & - & - & - & - & - & - & - & 27 & - & - & - & - & - \\
\hline Syrenia cana & - & - & - & - & - & - & - & - & - & - & 29 & - & - & - & - & - & - & - & - & - & 12 & - & - & - & 37 & - & - \\
\hline Crambe maritima & - & - & - & - & - & - & - & - & - & - & 27 & - & - & - & - & - & - & - & 17 & - & - & 30 & - & - & - & - & - \\
\hline Lappula squarrosa & - & - & - & - & - & - & - & - & - & - & - & 60 & - & 27 & - & - & - & - & - & - & - & - & - & - & - & - & - \\
\hline Lithospermum officinale & - & - & - & - & - & - & 16 & - & - & - & - & 33 & - & - & 51 & - & - & - & - & - & - & - & - & - & - & - & - \\
\hline Achillea asplenifolia & - & 10 & - & - & 18 & - & - & - & - & - & 13 & 28 & - & - & - & - & - & - & - & 26 & - & - & - & - & - & - & - \\
\hline Seseli tortuosum & - & - & - & - & 19 & - & - & - & - & - & - & 28 & - & - & - & 17 & - & - & 9 & 29 & - & - & - & - & - & - & - \\
\hline Polygonum arenarium s. arenarium & - & - & - & - & - & - & - & - & - & - & - & - & 73 & - & - & - & - & - & - & - & - & - & 39 & - & - & - & - \\
\hline Agropyron dasyanthum & - & - & - & - & - & - & - & - & - & - & - & - & 45 & - & - & - & - & - & - & - & 30 & - & - & 18 & 24 & 7 & - \\
\hline Anchusa gmelinii & - & - & - & - & - & - & - & - & - & - & - & - & 40 & - & - & - & - & - & - & - & 24 & - & - & - & 31 & - & - \\
\hline Syrenia montana & - & - & - & - & - & - & - & - & - & - & - & - & 32 & - & - & - & - & - & - & - & 22 & - & 38 & - & 15 & - & - \\
\hline Polygonum arenarium & - & - & - & - & - & - & - & - & - & - & - & - & - & 70 & - & - & - & - & - & - & 40 & - & - & - & - & - & - \\
\hline Veronica verna & - & - & - & - & - & - & - & - & - & - & - & - & - & 58 & - & - & 57 & - & - & - & - & - & - & - & - & - & - \\
\hline Centaurea apiculata s. adpressa & - & - & - & - & - & - & - & - & - & - & - & - & - & - & 37 & - & - & - & - & - & - & - & 44 & - & - & - & - \\
\hline Medicago sativa s. fal & - & - & - & - & 16 & - & - & - & - & - & 20 & - & - & - & 21 & 33 & - & - & - & 29 & - & - & 16 & - & - & - & - \\
\hline Gypsophila muralis & - & - & - & - & - & - & - & - & - & - & - & - & - & - & - & - & 43 & 41 & - & - & - & - & - & - & - & - & - \\
\hline Eryngium campestre & - & - & - & - & - & - & - & - & - & - & - & - & - & - & - & - & 34 & - & - & 36 & - & 23 & - & - & - & - & - \\
\hline Berteroa incana & - & - & - & - & - & - & - & - & - & - & - & - & - & - & - & - & 31 & 30 & - & - & - & 23 & - & - & - & - & - \\
\hline Chamaecytisus borysthenicus & - & - & - & 16 & - & - & - & - & - & - & - & - & - & - & - & - & - & 32 & - & - & 14 & - & - & 65 & - & - & - \\
\hline Hieracium echioides & - & - & - & - & - & - & - & - & - & - & - & - & - & - & - & - & - & 28 & - & - & 20 & - & - & 21 & 29 & - & - \\
\hline Centaurea margaritac & - & - & - & - & - & - & - & - & - & - & - & - & - & - & - & - & - & - & - & - & 60 & - & - & 32 & 25 & - & - \\
\hline Jurinea albicaulis $s$. & - & - & - & - & - & - & - & - & - & - & - & - & - & - & - & - & - & - & - & - & 47 & - & - & 42 & - & - & - \\
\hline Alyssum tortuosum & - & - & - & - & - & - & - & - & - & - & - & - & - & - & - & - & - & - & - & - & 43 & - & - & - & 40 & - & - \\
\hline Minuartia viscosa & - & - & - & - & - & - & - & - & - & - & - & - & - & - & - & - & - & - & - & - & 39 & - & - & 61 & 20 & - & - \\
\hline Thymus pallasiamus & - & - & - & - & - & - & - & - & - & - & - & - & - & - & - & - & - & - & - & - & 35 & - & - & 60 & - & - & 8 \\
\hline Cladina arbuscula & - & - & - & - & - & - & - & - & - & - & - & - & - & - & - & - & - & - & - & - & 34 & - & - & - & 72 & - & - \\
\hline Thymus borysthenicus & - & - & - & - & - & - & - & - & - & - & - & - & - & - & - & - & - & - & - & - & 31 & - & - & - & - & 36 & 25 \\
\hline Helichrysum arenarium & - & - & - & - & - & - & - & - & - & - & - & - & - & - & - & - & 23 & 17 & - & - & 25 & - & - & 27 & 23 & 8 & 13 \\
\hline Seseli arenarium & - & - & - & - & - & - & - & - & - & - & - & - & - & - & - & - & - & - & - & - & 12 & - & 57 & 12 & 44 & - & - \\
\hline Silene conica & - & - & - & - & - & - & - & - & - & - & - & - & - & - & - & - & - & - & - & - & 13 & - & 46 & - & 36 & - & - \\
\hline Herniaria polygama & - & - & - & - & - & - & - & - & - & - & - & - & - & - & - & - & - & - & - & - & 23 & - & - & 38 & 28 & - & - \\
\hline Cladonia subcariosa & - & - & - & - & - & - & - & - & - & - & - & - & - & - & - & - & - & - & - & - & 21 & - & - & 36 & 61 & - & - \\
\hline Cerastium semidecandrum & - & - & - & - & - & - & - & - & - & - & - & - & - & 15 & - & - & 13 & - & - & - & 10 & - & 24 & 35 & 35 & - & - \\
\hline Goniolimon graminifolium & - & - & - & - & - & - & - & - & - & - & - & - & - & - & - & - & - & - & - & - & 21 & - & - & 31 & 31 & - & - \\
\hline Scabiosa argentea & - & - & - & 17 & - & - & - & - & - & - & - & - & - & - & - & - & - & - & - & - & 25 & - & - & 31 & 42 & - & - \\
\hline Astragalus varius & - & - & - & - & 12 & - & - & - & - & - & 9 & - & - & - & - & - & 16 & - & - & - & 6 & - & - & 27 & 45 & - & - \\
\hline Dianthus platyodon & - & - & - & - & - & - & - & - & - & - & - & - & - & - & - & - & - & - & - & - & 22 & - & - & 26 & 26 & 12 & - \\
\hline Cerastium pumilum s. glutinosum & - & 35 & 16 & - & - & 58 & - & - & - & - & 26 & - & - & - & - & - & - & - & - & - & - & - & - & - & - & - & - \\
\hline Plantago lanceolata & - & - & - & - & - & 46 & - & - & - & - & 26 & - & - & - & - & - & 46 & - & - & - & - & - & - & - & - & - & - \\
\hline Bassia hirsuta & - & - & - & - & - & - & 40 & - & - & - & 53 & - & - & - & - & 29 & - & - & - & - & - & - & - & - & - & - & - \\
\hline chum acutum & - & - & 21 & - & - & - & 30 & - & - & - & 29 & - & - & - & - & 21 & - & - & - & 28 & - & - & - & - & - & - & - \\
\hline ium polium & - & - & - & - & 20 & - & 29 & - & - & - & 44 & - & - & - & - & 15 & - & - & - & 34 & - & - & - & - & - & - & - \\
\hline Bromus tectorum & - & - & - & - & - & - & 27 & 5 & 18 & - & 7 & 19 & 27 & 27 & - & - & 11 & - & - & - & - & 9 & - & - & - & - & - \\
\hline Plantago arenaria & - & - & - & - & - & - & - & - & - & - & - & - & 49 & 49 & 49 & - & - & - & - & - & - & - & - & - & - & - & - \\
\hline Erophila verna & - & - & - & - & - & - & - & - & - & - & - & - & - & - & - & - & 37 & - & - & - & 28 & - & - & - & 56 & - & - \\
\hline Myosotis stricta & - & - & - & - & - & - & - & - & - & - & - & - & - & - & - & - & 33 & - & - & - & 20 & - & - & 51 & 51 & - & - \\
\hline urea arenaria & - & - & - & - & 15 & - & - & - & - & - & - & - & - & - & - & - & - & - & 19 & 44 & - & 40 & 38 & - & - & - & - \\
\hline Bassia laniflora & - & - & - & - & - & - & - & - & - & - & - & - & - & - & - & - & - & - & - & - & 33 & - & - & 49 & 35 & - & - \\
\hline Veronica dillenii & - & - & - & - & - & - & - & - & - & - & - & - & - & - & - & - & - & - & - & - & 28 & - & - & 57 & 57 & - & - \\
\hline Tragopogon brevirostri & - & - & - & - & - & - & - & - & - & - & - & - & - & - & 23 & - & - & - & - & - & 28 & - & - & 38 & 44 & - & - \\
\hline ron cristatum & - & - & - & - & - & - & - & - & - & - & - & - & - & - & - & - & - & - & - & - & 28 & - & - & 26 & 73 & - & - \\
\hline borysthenic & - & - & - & - & - & - & - & - & - & - & - & - & 25 & - & - & - & - & - & - & - & 27 & - & - & 37 & 50 & - & - \\
\hline Cladonia foliacea & - & - & - & - & - & - & - & - & - & - & - & - & - & - & - & - & - & - & - & - & 21 & - & 47 & 47 & 47 & - & - \\
\hline Xanthopaemelia somloensis & - & - & - & - & - & - & - & - & - & - & - & - & - & - & - & - & - & - & - & - & 13 & - & 46 & 31 & 39 & - & - \\
\hline Alyssum minutum & - & - & - & - & - & - & - & - & - & - & - & - & - & - & - & - & - & - & - & - & 15 & - & 29 & 50 & 43 & - & - \\
\hline Cladonia rangiformis & - & - & - & - & - & - & - & - & - & - & - & - & - & - & - & - & - & - & - & - & 15 & - & 28 & 44 & 39 & - & 8 \\
\hline caulon aculeatum & - & - & - & - & - & - & - & - & - & - & - & - & - & - & - & - & - & - & - & - & 23 & - & 25 & 57 & 51 & - & - \\
\hline Centaurea diffusa & - & - & 5 & - & - & 45 & 33 & - & - & - & 30 & - & - & - & - & 14 & 29 & - & - & - & - & - & - & - & - & - & - \\
\hline Cynodon dactylon & 10 & 13 & - & - & 14 & 31 & 31 & - & - & 10 & 25 & - & - & - & 14 & 13 & - & - & - & 26 & - & - & - & - & - & - & - \\
\hline Artemisia campestris agg. & - & - & - & - & - & - & - & - & 28 & - & - & - & - & - & - & - & - & - & - & - & 28 & - & - & 28 & 28 & 10 & 19 \\
\hline Tortula ruralis & - & - & - & - & - & 18 & 10 & - & - & - & 29 & - & - & - & - & 11 & - & - & - & 18 & 11 & 10 & 29 & 29 & 29 & - & - \\
\hline la graveolens & - & - & - & - & - & - & - & - & - & - & - & - & 31 & - & - & - & - & - & - & - & 30 & - & - & 48 & 31 & - & - \\
\hline Koeleria glauca & - & 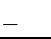 & - & - & - & - & - & - & - & - & - & - & - & 27 & - & - & - & 20 & - & - & 26 & - & 18 & 27 & 27 & - & 27 \\
\hline
\end{tabular}

Notes: only plant species having diagnostic value were listed; due to the table's width restriction, phi values were listed to integer, numbers mark syntaxa: 1 - Aperetum maritimae; 2 Dauco guttati-Chrysopogonetum grylli; 3 -Carici colchicae-Holoschoenetum vulgaris; 4 - Salici rosmarinifoliae-Holoschoenetum vulgaris; 5 -Secali-Stipetum borysthenicae; 6 Cynodonto-Medicaginetum minimae; 7-Secali-Cynodontetum dactyli; 8-Secaletum sylvestris; 9-Secali sylvestri-Brometum tectorum; 10 - Allio guttati-Festucetum rupicolae; 11 Heliotropio dolosi-Brometum japonici; 12 - Trago-Anthemietum ruthenicae, 13 - Linario odorae-Agropyretum dasyanthi; 14 - Linario odorae-Agropyretum dasyanthi (sensu Kovalenko); 15 - Plantaginetum arenariae; 16 - Poo bulbosae-Caricetum colchicae; 17 - Achilleo setacei-Festucetum beckeri; 18 - Centaureo gerberii-Chamaecytisetum borysthenici; 19 - Centaureo odessanae-Caricetum colchicae; 20 - Centaureo odessanae-Stipetum capillatae; 21 - Centaureo brevicipiti-Festucetum beckeri var. typica; 22 Centaureo odessanae-Festucetum beckeri (sensu Kolomiychuk et al.); 23 - Centaureo odessanae-Festucetum beckeri (Vicherek relevés); 24 - Centaureo brevicipiti-Festucetum beckeri salicetosum rosmarinifoliae; 25 - Centaureo brevicipiti-Festucetum beckeri imuletosum sabuletorum; 26 - Festucetum beckeri var. typica; 27 - Festucetum beckeri var. Koeleria glauca.

The class Festucetea vaginatae combines communities of sandy seppes and sandy meadows. In Ukraine, these coenoses are common in the steppe zone on the lower Dnieper River arenas, in the Black Sea region and

the Azov Sea regions. These coenoses are less common on riverine sands. According to our study, in Ukraine, the class includes one order, two alliances and 22 associations. Cluster analysis suggests that Artemisio arenariae- 
Festucion beckeri alliance is comprised of $4-5$ sub-alliances, but it is not still possible to identify clear diagnostic blocks (Fig. 5, Table 2). The leading factors of territorial differentiation of phytocoenoses also determining their coenotic diversity are the mesorelief patterns (elements of dunes, interdunal depressions, flat areas in arenas), soil type, its humus content and density, as well as the degree of aeolian processes influence. The hyperspace of ecological conditions from humid (deep depressions) to semi-desert (dune tops) contributes to their significant coenotic richness and diversity. The alliance Festucion beckeri includes coenoses occurring on sandy areas of low hilly coastal and riverine dunes which consist of non-carbonate soils. The alliance Artemisio arenariae-Festucion beckeri includes steppe plant communities occurring on sandy and sandy-shell soils of the Black Sea and the Azov Sea coastal areas.

According to the results obtained from ordination analysis of Festucetea vaginatae associations it was found that the ecological differentiation of syntaxa within the class is determined by the complex influence of ecologi- cal factors, and most of plant communities develop under similar environmental conditions (Fig. 6). The general leading factor is the soil salinity regime. Moisture, content of carbonates in the soil and its aeration are of crucial importance to the communities existing in Allio guttati-Festucetum rupicolae and Achilleo setacei-Festucetum beckeri associations. The distribution of Aperetum maritimae, Dauco guttati-Chrysopogonetum grylli and Carici colchicae-Holoschoenetum vulgaris coenoses in the ecological space is determined mainly by content of available nitrogen forms in the soil. Coenoses in the Secaletum sylvestris association are characterized by the widest ecological amplitude. Their placement is determined by the variability of damping and temperature conditions.

Coenoses belonging to Helichryso-Crucianelletea maritimae class occupy specific ecotopes in stabilized overgrown (grey) dunes and overgrown uprush berm crest areas on the coasts of the Black Sea and Azov Sea. In Ukraine, the class includes 1 order, 4 alliances and 10 associations (Fig. 7, Table 3).

Table 3

Synoptic table of Helichryso-Crucianelletea maritimae class

\begin{tabular}{|c|c|c|c|c|c|c|c|c|c|c|}
\hline No. of syntaxa & 1 & 2 & 3 & 4 & 5 & 6 & 7 & 8 & 9 & 10 \\
\hline Number of relevés & 13 & 10 & 7 & 10 & 24 & 14 & 10 & 14 & 17 & 9 \\
\hline Onopordum tauricum & 60.0 & - & - & - & - & - & - & - & - & - \\
\hline Matricaria perforata & 59.2 & - & - & - & - & - & - & - & - & - \\
\hline Poa angustifolia & 55.1 & - & - & - & - & - & - & - & - & - \\
\hline Cynanchum acutum & 54.5 & - & - & - & - & - & - & - & - & - \\
\hline Seseli tortuosum & 50.8 & - & - & - & - & - & - & - & - & - \\
\hline Falcaria vulgaris & 46.9 & - & - & - & - & - & - & - & - & - \\
\hline Cerastium gracile & 46.3 & - & - & - & - & - & - & - & - & - \\
\hline Allium flavum s. tauricum & 46.3 & - & - & - & - & - & - & - & - & - \\
\hline Berteroa incana & 45.0 & - & - & - & - & - & - & - & - & - \\
\hline Camelina microcarpa & 41.3 & - & - & - & - & - & - & - & - & - \\
\hline Bromus squarrosus & 40.7 & - & - & - & - & - & - & - & - & - \\
\hline Bassia laniflora & 39.5 & - & - & - & - & - & - & - & - & - \\
\hline Allium scorodoprasum s. waldsteinii & 37.5 & - & - & - & - & - & - & - & - & - \\
\hline Phragmites australis & 37.5 & - & - & - & - & - & - & - & - & - \\
\hline Galium humifusum & 35.3 & - & - & - & - & - & - & - & - & - \\
\hline Crambe maritima & 32.8 & - & - & - & - & - & - & - & - & - \\
\hline Alyssum desertorum & 32.4 & - & - & - & - & - & - & - & - & - \\
\hline Odontites verna s. serotina & 29.5 & - & - & - & - & - & - & - & - & - \\
\hline Elymus repens & 28.8 & - & - & - & - & - & - & - & - & - \\
\hline Helichrysum arenarium & - & 51.1 & - & 20.0 & - & - & - & - & - & - \\
\hline Veronica verna & - & 42.9 & - & - & - & - & - & - & - & - \\
\hline Arenaria serpyllifolia s. leptoclados & - & 40.8 & - & - & - & - & - & - & - & - \\
\hline Silene spergulifolia & - & 35.0 & - & - & - & - & - & - & - & - \\
\hline Conyza canadensis & - & 26.3 & - & - & - & - & - & - & - & - \\
\hline Scabiosa argentea & - & - & 86.2 & 16.8 & - & - & - & - & - & - \\
\hline Lithospermum officinale & - & - & 57.5 & - & - & - & - & - & - & - \\
\hline Centaurea margaritacea s. breviceps & - & - & 51.4 & - & - & - & - & - & - & - \\
\hline Medicago minima & - & - & 49.1 & - & - & - & - & - & - & 21.2 \\
\hline Gypsophila paniculata & - & - & 35.5 & - & - & - & - & - & - & - \\
\hline Asperula setulosa & - & - & - & 100.0 & - & - & - & - & - & - \\
\hline Seseli arenarium & - & - & - & 100.0 & - & - & - & - & - & - \\
\hline Artemisia tschernieviana & - & - & - & 87.5 & - & - & - & - & - & - \\
\hline Elymus farctus & - & - & - & 82.3 & - & - & - & - & - & - \\
\hline Cerastium semidecandrum & - & - & - & 69.2 & - & - & - & - & - & - \\
\hline Silene conica & - & - & - & 68.8 & - & - & - & - & - & - \\
\hline Alyssum minutum & - & - & - & 68.8 & - & - & - & - & - & - \\
\hline Syrenia montana & - & 22.3 & - & 58.9 & - & - & - & - & - & - \\
\hline Onosma arenaria & - & - & - & 55.5 & - & - & - & - & - & - \\
\hline Polygonum arenarium s. arenarium & 20.2 & - & - & 54.7 & - & - & - & - & - & - \\
\hline Orobanche coerulescens & - & - & - & 45.9 & - & - & - & - & - & - \\
\hline Koeleriaglauca & - & - & - & 43.0 & - & - & - & 14.2 & - & - \\
\hline Asparagus officinalis s. officinalis & - & - & - & 42.9 & - & - & - & - & - & - \\
\hline Festuca beckeri & - & - & - & 42.8 & - & - & - & - & 14.5 & - \\
\hline Linum perenne & - & - & - & 37.2 & - & - & - & - & - & - \\
\hline Picris hieracioides & - & - & - & 37.2 & - & - & - & - & - & - \\
\hline Centaurea arenaria s. odessana & 15.0 & - & - & 34.4 & - & - & - & 16.9 & - & - \\
\hline Echinops sphaerocephalus & - & - & - & - & 39.1 & - & - & - & - & - \\
\hline Galium ruthenicum & - & - & - & - & 33.8 & - & - & - & - & - \\
\hline Centaurea arenaria s. borysthenica & - & - & - & - & 33.8 & - & - & - & - & - \\
\hline Glycyrrhiza glabra & - & - & - & - & 27.5 & - & - & - & - & - \\
\hline Consolida regalis & - & - & - & - & 27.5 & - & - & - & - & - \\
\hline Seseli campestre & - & - & - & - & - & 87.7 & - & - & - & - \\
\hline Asperula graveolens & - & - & - & - & - & 78.6 & - & - & - & - \\
\hline Galium tenderiense & - & - & - & - & - & 74.3 & - & - & - & - \\
\hline Melica transsilvanica s. klokovii & - & - & - & - & - & 63.5 & - & - & - & - \\
\hline Limonium meyeri & - & - & - & - & - & 56.2 & - & - & - & - \\
\hline Anchusa gmelinii & - & - & - & - & - & 51.4 & - & - & - & - \\
\hline Elymus elongatus & - & - & - & - & - & 51.4 & - & - & - & - \\
\hline Eleocharis palustris & - & - & - & - & - & 44.4 & - & - & - & - \\
\hline Cuscuta monogyna & - & - & - & - & - & 44.4 & - & - & - & - \\
\hline
\end{tabular}




\begin{tabular}{|c|c|c|c|c|c|c|c|c|c|c|}
\hline No. of syntaxa & 1 & 2 & 3 & 4 & 5 & 6 & 7 & 8 & 9 & 10 \\
\hline Number of relevés & 13 & 10 & 7 & 10 & 24 & 14 & 10 & 14 & 17 & 9 \\
\hline Linaria dulcis & - & - & - & - & - & 39.8 & - & - & - & - \\
\hline Papaver dubium s. laevigatum & - & - & - & - & - & 36.7 & - & - & - & - \\
\hline Consolida paniculata & - & - & - & - & - & 36.3 & - & - & - & - \\
\hline Artemisia scoparia & - & - & - & - & - & 36.1 & - & - & - & - \\
\hline Scabiosa ochroleuca & - & - & - & - & - & 36.1 & - & - & - & - \\
\hline Senecio vernalis & - & - & - & - & - & 31.5 & - & - & - & 24.7 \\
\hline Bromus tectorum & - & 24.2 & - & - & - & 30.9 & - & 21.3 & - & - \\
\hline Apera spica-venti s. maritima & - & - & - & - & - & 26.4 & - & - & - & - \\
\hline Aster oleifolius & - & - & - & - & - & - & 61.2 & - & - & - \\
\hline Echium vulgare & - & - & - & - & - & - & 56.6 & 15.5 & - & - \\
\hline Anthemis cotula & - & - & - & - & - & - & 52.8 & - & - & - \\
\hline Limonium latifolium & - & - & - & - & - & - & 49.0 & - & 17.0 & - \\
\hline Dianthus marschallii & - & - & - & - & - & - & 47.3 & - & - & - \\
\hline Goniolimon tataricum & - & - & - & - & - & - & 43.5 & - & - & - \\
\hline Helianthemum salicifolium & - & - & - & - & - & - & 43.0 & - & - & - \\
\hline Viola kitaibeliana & - & - & - & - & - & - & 42.9 & - & - & - \\
\hline Salvia aethiopis & - & - & - & - & - & - & 42.9 & - & - & - \\
\hline Scorzonera cana & - & - & - & - & - & - & 42.9 & - & - & - \\
\hline Peganum harmala & - & - & - & - & - & - & 42.9 & - & - & - \\
\hline Petrorhagia prolifera & - & - & - & - & - & - & 42.9 & - & - & - \\
\hline Centaurea orientalis & - & - & - & - & - & - & 42.9 & - & - & - \\
\hline Capsella bursa-pastoris & - & - & - & - & - & - & 42.9 & - & - & - \\
\hline Satureja rumelica & - & - & - & - & - & - & 42.9 & - & - & - \\
\hline Potentilla astracanica & - & - & - & - & - & - & 39.5 & - & - & - \\
\hline Valerianella pumila & - & - & - & - & - & - & 32.0 & - & - & - \\
\hline Asperula supina & - & - & - & - & - & - & - & 91.9 & - & - \\
\hline Euphorbia esula s. tommasiniana & - & - & - & - & - & - & - & 91.9 & - & - \\
\hline Carex liparocarpos & - & - & - & - & - & - & - & 90.1 & - & - \\
\hline Linaria sabulosa & - & - & - & - & - & - & - & 85.4 & - & - \\
\hline Thymus pannonicus & - & - & - & - & - & - & - & 84.4 & - & - \\
\hline Clypeola jonthlaspi & - & - & - & - & - & - & - & 83.2 & - & - \\
\hline Medicago praecox & - & - & - & - & - & - & - & 78.6 & - & - \\
\hline Trigonella monspeliaca & - & - & - & - & - & - & - & 77.3 & - & - \\
\hline Eragrostis minor & - & - & - & - & - & - & - & 69.7 & - & - \\
\hline Erysimum diffusum & - & - & - & - & - & - & - & 66.5 & - & 23.9 \\
\hline Arenaria serpyllifolia & - & - & - & - & - & - & - & 63.4 & - & - \\
\hline Stipa capillata & - & - & - & - & - & - & - & 63.1 & - & - \\
\hline Alcearugosa & - & - & - & - & - & - & - & 62.6 & - & - \\
\hline Sisymbrium orientale & - & - & - & - & - & - & - & 61.8 & - & - \\
\hline Convolvulus lineatus & - & - & - & - & - & - & - & 60.1 & - & - \\
\hline Erodium cicutarium & - & - & - & - & - & - & - & 60.1 & - & - \\
\hline Setaria viridis & - & - & - & - & - & - & - & 59.0 & 20.8 & - \\
\hline Thesium arvense & - & - & - & - & - & - & - & 58.9 & - & - \\
\hline Leontodon hispidus & - & - & - & - & - & - & - & 52.7 & - & - \\
\hline Lappula barbata & - & - & - & - & - & - & - & 51.7 & - & - \\
\hline Achillea leptophylla & - & - & - & - & - & - & - & 50.9 & - & - \\
\hline Psilurus incurvus & - & - & - & - & - & - & - & 49.1 & - & - \\
\hline Minuartia hybrida & - & - & - & - & - & - & - & 48.5 & - & - \\
\hline Hieracium umbellatum & - & - & - & - & - & - & - & 48.5 & - & - \\
\hline Tribulus terrestris & - & - & - & - & - & - & - & 45.9 & - & - \\
\hline Artemisia taurica & - & - & - & - & - & - & - & 44.4 & - & - \\
\hline Minuartia glomerata & - & - & - & - & - & - & - & 44.4 & - & - \\
\hline Erophila verna s. praecox & - & - & - & - & - & - & - & 42.7 & - & - \\
\hline Cerastium pumilum s. glutinosum & - & - & - & - & - & - & - & 41.9 & - & - \\
\hline Plantago lanceolata & - & - & - & - & - & - & 24.0 & 41.6 & - & - \\
\hline Silene conica s. subconica & 18.8 & - & - & - & - & - & - & 39.7 & - & 24.6 \\
\hline Papaver rhoeas & - & - & - & - & - & - & - & 38.0 & 20.7 & - \\
\hline Heliotropium ellipticum & - & - & - & - & - & - & - & 36.1 & - & - \\
\hline Cerastium fontanum s. vulgare & - & - & - & - & - & - & - & 36.1 & - & - \\
\hline Artemisia austriaca & - & - & - & - & - & - & - & 34.7 & - & - \\
\hline Artemisia campestris agg. & - & - & - & - & - & - & - & 33.0 & - & 21.1 \\
\hline Centaurea diffusa & - & - & - & - & - & - & - & 28.9 & - & - \\
\hline Salvia scabiosifolia & - & - & - & - & - & - & - & - & 66.7 & - \\
\hline Centaurea sterilis & - & - & - & - & - & - & - & - & 62.2 & - \\
\hline Apera spica-venti & - & - & - & - & - & - & - & - & 57.4 & - \\
\hline Crataegus laciniata & - & - & - & - & - & - & - & - & 52.2 & - \\
\hline Rosa canina & - & - & - & - & - & - & - & - & 52.2 & - \\
\hline Adonis aestivalis & - & - & - & - & - & - & - & - & 52.2 & - \\
\hline Melica ciliata s. monticola & - & - & - & - & - & - & - & - & 46.6 & - \\
\hline Koeleria macrantha & - & - & - & - & - & - & - & - & 46.6 & - \\
\hline Festuca rupicola & - & - & - & - & - & - & - & - & 46.6 & - \\
\hline Arum elongatum & - & - & - & - & - & - & - & - & 40.2 & - \\
\hline Anthriscus cerefolium & - & - & - & - & - & - & - & - & 40.2 & - \\
\hline Sambucus nigra & - & - & - & - & - & - & - & - & 40.2 & - \\
\hline Bromus hordeaceus s. hordeaceus & - & - & - & - & - & - & - & - & 40.2 & - \\
\hline Alyssum calycocarpum & - & - & - & - & - & - & - & - & 40.2 & - \\
\hline Trinia crithmifolia & - & - & - & - & - & - & - & - & 40.2 & - \\
\hline Geum urbanum & - & - & - & - & - & - & - & - & 40.2 & - \\
\hline Valerianella coronata & - & - & - & - & - & - & - & - & 40.2 & - \\
\hline Dactylis glomerata & - & - & - & - & - & - & - & - & 40.2 & - \\
\hline Galium aparine & - & - & - & - & - & - & - & - & 40.2 & - \\
\hline Milium vernale & - & - & - & - & - & 24.8 & - & - & 36.9 & - \\
\hline Agropyron cristatum s. ponticum & - & - & - & - & - & - & - & - & 32.7 & - \\
\hline Thalictrum minus & - & - & - & - & - & - & - & - & 32.7 & - \\
\hline
\end{tabular}




\begin{tabular}{|c|c|c|c|c|c|c|c|c|c|c|}
\hline No. of syntaxa & 1 & 2 & 3 & 4 & 5 & 6 & 7 & 8 & 9 & 10 \\
\hline Number of relevés & 13 & 10 & 7 & 10 & 24 & 14 & 10 & 14 & 17 & 9 \\
\hline Dactylis glomerata s. hispanica & - & - & - & - & - & - & - & - & 32.7 & - \\
\hline Buglossoides arvensis & - & - & - & - & - & - & - & - & 32.5 & - \\
\hline Lactuca tatarica & - & - & - & - & - & - & - & - & 31.9 & - \\
\hline Carex ligerica & - & - & - & 23.4 & - & - & - & - & 30.1 & - \\
\hline Agropyron cimmericum & - & - & - & - & - & - & - & - & 27.1 & - \\
\hline Potentilla species & - & - & - & - & - & - & - & - & - & 76.3 \\
\hline Linaria genistifolia s. genistifolia & - & - & - & - & - & - & - & - & - & 64.7 \\
\hline Asperula tenella & - & - & - & - & - & - & - & - & - & 64.7 \\
\hline Onosma tinctoria & - & - & - & - & - & - & - & - & - & 60.1 \\
\hline Cerastium pumilum & - & - & - & - & - & - & - & - & - & 60.1 \\
\hline Medicago lupulina & - & - & - & - & - & - & - & - & - & 55.7 \\
\hline Cichorium intybus & - & - & - & - & - & - & - & - & - & 49.8 \\
\hline Elymus farctus s. bessarabicus & - & - & - & - & - & - & - & - & - & 49.8 \\
\hline Holosteum umbellatum & - & - & - & - & - & - & - & - & - & 45.2 \\
\hline Crepis foetida s. rhoeadifolia & - & - & - & - & - & - & - & 24.5 & - & 43.7 \\
\hline Hieracium echioides & - & - & - & - & - & - & - & - & 19.0 & 42.8 \\
\hline Astrodaucus littoralis & - & - & - & - & - & - & - & - & - & 30.1 \\
\hline Coronilla varia & - & - & - & - & - & - & - & 18.3 & - & 26.3 \\
\hline Artemisia santonicum & 57.2 & - & - & - & - & 25.6 & - & - & - & - \\
\hline Silene exaltata & 53.3 & - & - & 54.0 & - & - & - & - & - & - \\
\hline Medicago sativa s. falcata & 33.5 & - & - & - & - & - & - & 23.9 & - & 26.0 \\
\hline Gypsophila perfoliata & 30.8 & 25.8 & - & - & - & - & - & - & - & - \\
\hline Potentilla argentea & 30.8 & 25.8 & - & - & - & - & - & - & - & - \\
\hline Linaria genistifolia & 29.5 & - & - & 43.2 & - & - & - & - & - & - \\
\hline Leymus racemosus s. sabulosus & - & 47.0 & - & - & 32.9 & - & - & - & - & - \\
\hline Eryngium maritimum & - & 32.6 & - & - & - & - & - & - & - & 48.2 \\
\hline Melilotus alba & - & 28.7 & - & - & - & - & - & - & - & 56.0 \\
\hline Syrenia cana & - & - & 50.6 & - & - & 27.3 & - & - & - & - \\
\hline Anthemis ruthenica & - & - & 41.8 & - & - & - & 38.3 & - & - & - \\
\hline Festuca valesiaca & - & - & 39.7 & - & - & - & - & 33.0 & - & - \\
\hline Alyssum borzaeanum & - & - & - & 64.4 & - & 58.6 & - & - & - & - \\
\hline Ephedra distachya & - & - & - & - & 34.3 & 34.3 & - & - & - & - \\
\hline Centaurea apiculata s. adpressa & - & - & - & 24.8 & - & 30.8 & - & 30.8 & - & - \\
\hline Ajuga chamaepitys s. chia & - & - & - & - & - & - & 71.8 & 46.9 & - & - \\
\hline Marrubium peregrinum & - & - & - & - & - & - & 53.9 & 32.6 & - & - \\
\hline Cynodon dactylon & - & - & - & - & - & - & 47.1 & 47.1 & - & - \\
\hline Linum austriacum s. euxinum & - & - & - & - & - & - & 42.3 & 50.0 & - & - \\
\hline Rumex tuberosus & - & - & - & - & - & - & 39.5 & - & 33.7 & - \\
\hline Echinops ritro & - & - & - & - & - & - & 37.3 & - & 46.6 & 23.6 \\
\hline Eryngium campestre & - & - & - & - & - & - & 36.9 & 41.1 & - & - \\
\hline Carduus uncinatus & - & - & - & - & - & - & 35.5 & 58.8 & - & 24.3 \\
\hline Potentilla taurica & - & - & - & - & - & - & 27.8 & 66.8 & - & - \\
\hline Silene densiflora & - & - & - & - & - & - & 26.2 & 50.4 & - & - \\
\hline Sideritis montana s. montana & - & - & - & - & - & - & - & 60.8 & - & 57.5 \\
\hline Thymus dimorphus & - & - & - & - & - & - & - & 53.4 & - & 59.0 \\
\hline Teucrium polium & - & - & - & - & - & - & - & 52.8 & - & 28.2 \\
\hline Silene syreistschikowii & - & - & - & - & - & - & - & 47.5 & - & 39.2 \\
\hline Elymus uralensis s. viridiglumis & - & - & - & - & - & - & - & 45.3 & 20.0 & 26.9 \\
\hline Stipa borysthenica & - & - & - & 21.9 & - & - & - & 37.6 & 34.4 & - \\
\hline Allium guttatum & - & - & - & - & - & - & - & 33.0 & - & 27.8 \\
\hline Alyssum tortuosum & - & - & - & - & - & - & - & 29.5 & - & 44.4 \\
\hline Plantago arenaria & - & - & - & - & - & - & - & 26.9 & - & 43.2 \\
\hline Verbascum pinnatifidum & - & - & - & - & - & - & - & 23.3 & 46.8 & 55.7 \\
\hline Jurinea albicaulis s. laxa & - & - & - & - & - & - & - & - & 39.9 & 57.3 \\
\hline Leymus racemosus & - & - & - & - & - & - & - & - & 33.3 & 65.8 \\
\hline Astragalus onobrychis & 26.1 & - & - & - & - & - & - & - & 33.8 & 41.9 \\
\hline Alyssum turkestanicum & - & - & - & - & - & - & - & 54.9 & 41.7 & 29.9 \\
\hline Alyssum hirsutum & - & - & - & - & - & - & - & 44.8 & 37.3 & 25.9 \\
\hline Chondrilla juncea & - & - & - & - & - & - & - & 33.6 & 40.5 & 33.1 \\
\hline Astragalus varius & - & - & - & - & - & - & 23.7 & 26.8 & 31.5 & 36.8 \\
\hline Secalesylvestre & - & - & - & - & - & - & - & - & 24.4 & 20.9 \\
\hline Euphorbia seguierana & - & - & - & 19.6 & - & - & - & 23.4 & - & - \\
\hline Astragalus cicer & - & - & - & - & 23.0 & - & - & - & - & - \\
\hline Melica ciliata & - & - & - & - & - & - & - & - & 23.8 & - \\
\hline Xanthium strumarium s. italicum & - & - & - & - & - & - & - & - & 21.9 & - \\
\hline Poabulbosa & - & - & - & - & - & - & - & 15.7 & 13.5 & - \\
\hline
\end{tabular}

Notes: numbers mark syntaxa: 1 - Anisantho tectorum-Medicaginetum kotovii; 2 - Anisantho tectorum-Helichrysetum arenariae; 3 - Scabioso ucranicae-Caricetum ligericae; 4 - Secali sylvestri-Alyssetum borzaeani; 5 - Ephedro-Caricetum colchicae; 6 -Medicagini tenderiensis-Seselietum tenderiensi; 7 - Cynodonto-Ajugetum chiae; 8 - Carici liparicarpo-Centaureetum adpressae; 9 -Astragalo borysthenici-Ephedretum distachyae; 10 -Leymo-Verbascetum pinnatifidi. 


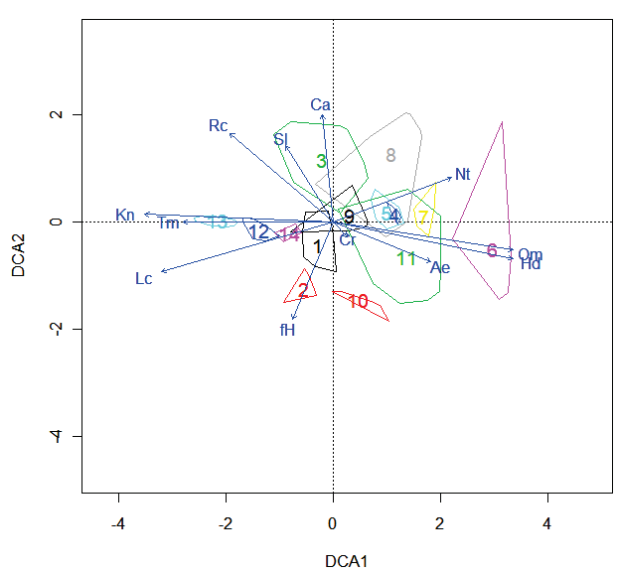

Fig. 4. Results of ordination analysis of syntaxa of the class KoelerioCorynephoretea canescentis; numbers mark syntaxa: 1 - Veronico dillenii-Secaletum sylvestris; 2 -Chamaecytiso ruthenicae-Festucetum beckeri; 3 -Diantho deltoidis-Armerietum elongatae; 4-Jasiono montanae-Thymetum serpylli; 5 -Jasiono montanae-Festucetum ovinae; 6-Corynephoro-Silenetum tataricae; 7-Artemisio campestris-

Dianthetum borbasii; 8 - Artemisio dniproicae-Sedetum sexangularis; 9 -

Veronico dillenii-Secaletum sylvestris (sensu Kovalenko); 10 -Diantho borbasii-Agrostietum syreistschikovii; 11 - Thymo angustifolii-

Festucetum beckeri; 12 - Centaureo borysthenicae-Festucetum beckeri; 13 - Veronico dillenii-Corynephoretum; 14 - Corniculario aculeataeCorynephoretum canescentis; here and further the scale of ecological factors developed by Didukh (2011) was used: $\mathrm{Hd}$ - soil moisture; $\mathrm{Nt}$ content of available nitrogen forms in the soil; $\mathrm{Ca}$-content of carbonates; $\mathrm{Rc}$ - soil acidity; $\mathrm{Sl}$ - salt regime; $\mathrm{fH}$ - soil moisture variability; $\mathrm{Ae}$ - soil aeration; $\mathrm{Lc}$ - light intensity; $\mathrm{Kn}$ - climate continentality; $\mathrm{Om}$ ombroregime; $\mathrm{Tm}$ - temperature regime; $\mathrm{Cr}$ - cryoregime; DCA1, DCA2 - ordination axes

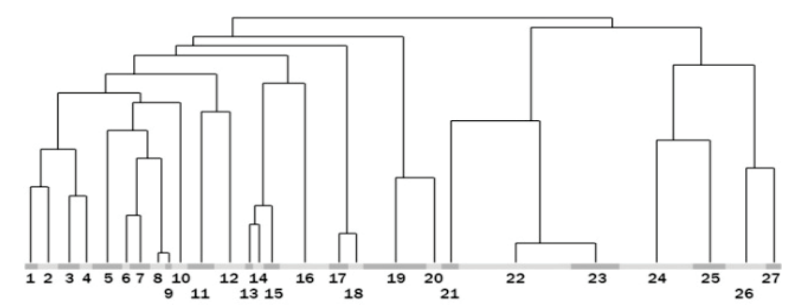

Fig. 5. Cluster analysis of phytosociological data of the Festucetea vaginatae class; clusters: 1 -Aperetum maritimae; 2 -Dauco guttatiChrysopogonetum grylli; 3 -Carici colchicae-Holoschoenetum vulgaris; 4-Salici rosmarinifoliae-Holoschoenetum vulgaris; 5-Secali-Stipetum borysthenicae; 6-Cynodonto-Medicaginetum minimae; 7 -SecaliCynodontetum dactyli; 8 -Secaletum sylvestris; 9 - Secali sylvestriBrometum tectorum; 10 - Allio guttati-Festucetum rupicolae; 11 Heliotropio dolosi-Brometum japonici; 12 -Trago-Anthemietum ruthenicae; 13 -Linario odorae-Agropyretum dasyanthi; 14 -Linario odorae-Agropyretum dasyanthi (sensu Kovalenko); 15 - Plantaginetum arenariae; 16 -Poo bulbosae-Caricetum colchicae; 17 -Achilleo setacei-

Festucetum beckeri;18 - Centaureo gerberii-Chamaecytisetum borysthenici; 19-Centaureo odessanae-Caricetum colchicae; 20 Centaureo odessanae-Stipetum capillatae; 21 - Centaureo brevicipitiFestucetum beckeri var. typica; 22 - Centaureo odessanae-Festucetum beckeri (sensu Kolomiychuk); 23 -Centaureo odessanae-Festucetum beckeri (Vicherek relevés); 24-Centaureo brevicipiti-Festucetum beckeri salicetosum rosmarinifoliae; 25 -Centaureo brevicipiti-Festucetum beckeri inuletosum sabuletorum; 26-Festucetum beckeri var. typica; 27 - Festucetum beckeri var. Koeleria glauca

The Ephedro distachyae-Medicaginion romanicae alliance includes plant communities of stabilized coastal dunes and uprush berm crest areas covered with dense sand-shell soils in the Black Sea and Azov Sea. The Medicagini tenderiensis-Seselion tenderiensi alliance is presented by coenoses of mesophytic sand steppes on accumulative sand-shell macroforms in the northwest coast of the Black Sea. The Scabiosion ucranicae alliance is presented by coenoses of stabilized coastal dunes in the Western Black Sea Region. The Cynodonto-Teucrion polii alliance includes plant communities on young dunes, as well as on uprush limit parts of berm crest areas growing on washed sandy and shelly soils, enriched with detritus, on the Azov-Black Sea coast of Crimea.

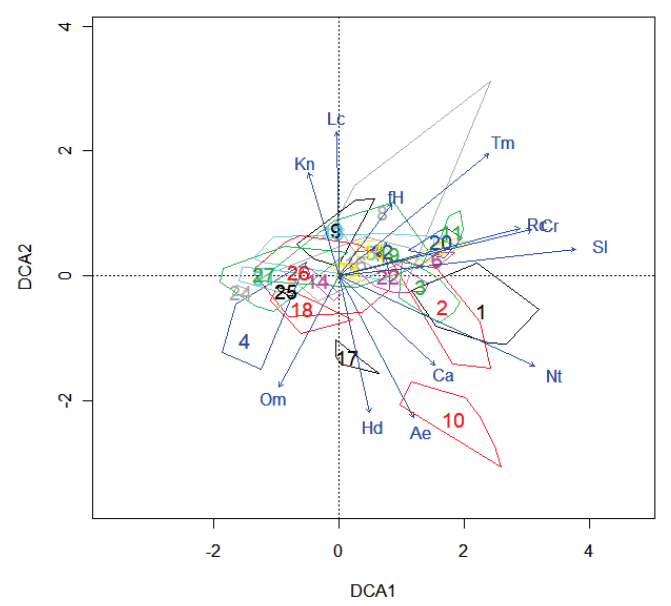

Fig. 6. Results of ordination analysis obtained for syntaxa of Festucetea vaginatae class; numbers mark syntaxa: 1 - Aperetum maritimae; 2-Dauco guttati-Chrysopogonetum grylli; 3 - Carici colchicaeHoloschoenetum vulgaris; 4 -Salici rosmarinifoliae-Holoschoenetum vulgaris; 5 -Secali-Stipetum borysthenicae; 6-Cynodonto-

Medicaginetum minimae; 7 -Secali-Cynodontetum dactyli; 8 -Secaletum sylvestris; 9 - Secali sylvestri-Brometum tectorum; 10 - Allio guttati-

Festucetum rupicolae; 11 - Heliotropio dolosi-Brometum japonici; 12 Trago-Anthemietum ruthenicae; 13 - Linario odorae-Agropyretum dasyanthi (Vicherek relevés); 14-Linario odorae-Agropyretum dasyanthi (sensu Kovalenko); 15 - Plantaginetum arenariae; 16-Poo bulbosae-Caricetum colchicae; 17 -Achilleo setacei-Festucetum beckeri; 18 -Centaureo gerberii-Chamaecytisetum borysthenici; 19 - Centaureo odessanae-Caricetum colchicae; 20 - Centaureo odessanae-Stipetum capillatae; 21 - Centaureo brevicipiti-Festucetum beckeri var. typica; 22 Centaureo odessanae-Festucetum beckeri (sensu Kolomiychuk et al.); 23 -Centaureo odessanae-Festucetum beckeri (Vicherek relevés); 24 -

Centaureo brevicipiti-Festucetum beckeri salicetosum rosmarinifoliae; 25 - Centaureo brevicipiti-Festucetum beckeri inuletosum sabuletorum; 26-Festucetum beckeri var. typica; 27-Festucetum beckeri var. Koeleria glauca

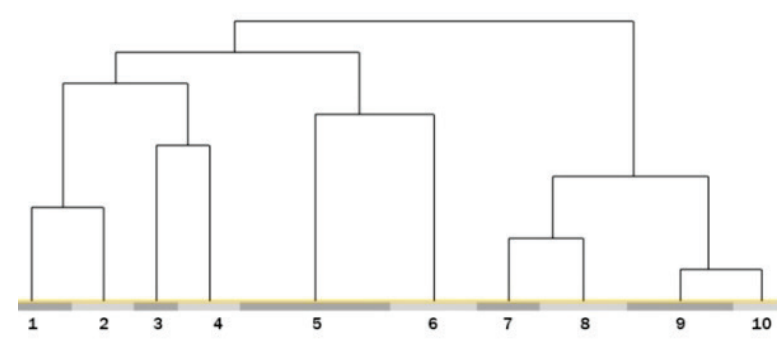

Fig. 7. Cluster analysis of phytosociological data of HelichrysoCrucianelletea maritimae class: 1 -Anisantho tectorum-Medicaginetum kotovii; 2 -Anisantho tectorum-Helichrysetum arenariae; 3 -Scabioso ucranicae-Caricetum ligericae; 4 -Secali sylvestri-Alyssetum borzaeani;

5-Ephedro-Caricetum colchicae; 6-Medicagini tenderiensis-

Seselietum tenderiensi; 7 -Cynodonto-Ajugetum chiae; 8-Carici

liparicarpo-Centaureetum adpressae; 9 - Astragalo borysthenici-

Ephedretum distachyae; 10 - Leymo-Verbascetum pinnatifidi

The leading factors of territorial differentiation of these communities are the landforms and their relative age, groundwater level, substrate density and type (sandy, shelly), as well as the degree of dune geomorphostructures stabilization depending on the activity of the sea and aeolian processes. 


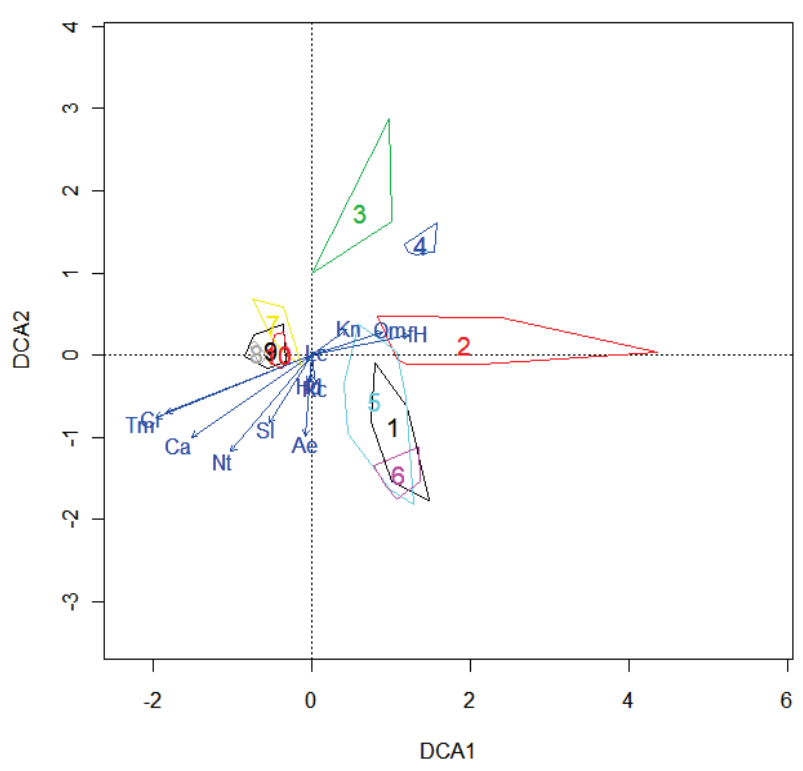

Fig. 8. Results obtained from ordination analysis of associations in the class Helichryso-Crucianelletea maritimae: 1 -Anisantho tectorumMedicaginetum kotovii; 2 -Anisantho tectorum-Helichrysetum arenariae; 3 -Scabioso ucranicae-Caricetum ligericae; 4 -Secali sylvestriAlyssetum borzaeani; 5-Ephedro-Caricetum colchicae; 6-Medicagini tenderiensis-Seselietum tenderiensi; 7 - Cynodonto-Ajugetum chiae; 8 Carici liparicarpo-Centaureetum adpressae; 9-Astragalo borystheniciEphedretum distachyae; 10 -Leymo-Verbascetum pinnatifidi

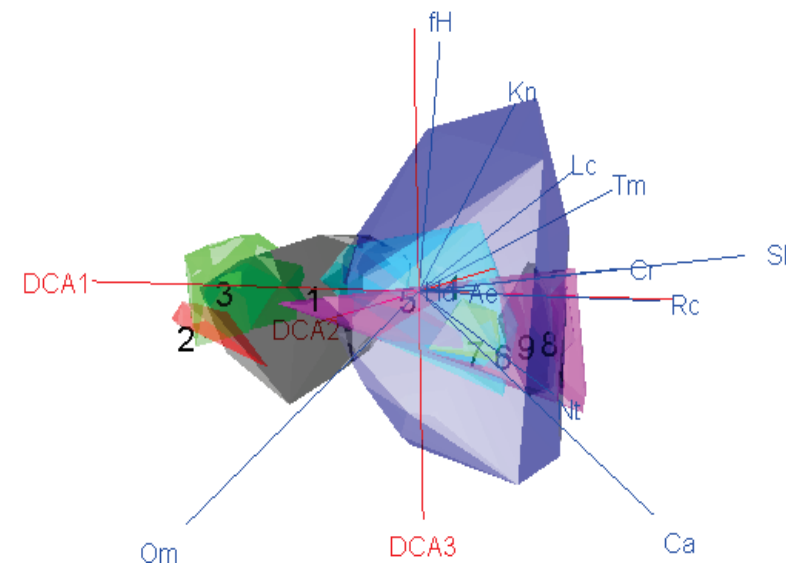

Fig. 9. Results of ordination analysis obtained for the classes Festucetea vaginatae, Koelerio-Corynephoretea canescentis and HelichrysoCrucianelletea maritimae by alliances: numbers mark alliances:

1 -Koelerion glaucae; 2 -Armerion elongatae; 3 - Corynephorion canescentis; 4-Artemisio arenariae-Festucion beckeri; 5-Festucion

beckeri; 6-Ephedro distachyae-Medicaginion romanicae; 7 Scabiosion ucranicae; 8 -Medicagini tenderiensis-Seselion tenderiensi; 9-Cynodonto-Teucrion polii

Within the ecological space, distribution of communities is mainly determined by the variability of damping, ombroregime and climate continentality factors (Fig. 8). They have a greater influence on the coenoses in Anisantho tectorum-Helichrysetum arenariae, Scabioso ucranicae-Caricetum ligericae and Secali sylvestri-Alyssetum borzaeani associations. In addition to these factors, Anisantho tectorumMedicaginetum kotovii, Ephedro-Caricetum colchicae and Medicagini tenderiensis-Seselietum tenderiensi communities also depend on the degree of soil aeration, soil humidity and the content of various salts. Anisantho tectorum-Helichrysetum arenariae, Scabioso ucranicaeCaricetum ligericae and Ephedro-Caricetum colchicae syntaxa have greater stenotopy, while coenoses of Cynodonto-Teucrion polii alliance developing in the Crimea demonstrate stenotopy by most ecological parameters.

\section{Discussion}

Studies on the vegetation cover of natural zones in Eurasia are important for understanding the processes of their development and functioning at the continental scale (Erdös et al., 2018). Therefore, a critical analysis of the accumulated phytocoenological materials against the background of modern knowledge is important. At the present time, the syntaxonomic content of the classes Festucetea vaginatae and KoelerioCorynephoretea canescentis is still a debatable issue, which is facilitated by the transitional position of their syntaxa and the belonging of certain diagnostic species of the highest classification ranks to the same genus, in particular, coenose-forming species Festuca psammophila, F. polesica, $F$. vaginata, and $F$. beckeri. As already noted, a number of European authors classified phytocoenoses of overgrown sands as belonging to the order Festucetalia vaginatae Soó 1957 in the class Koelerio-Corynephoretea canescentis (Dring et al., 2002; Borhidi, 2003; Biondi et al., 2014), when others classified them as entering into the class Festuco-Brometea (Rodwell et al., 2002; Tzonev et al., 2009). Complementary to Ukraine, scientists from the Czech Republic (Chytrý, 2007), Romania (Sanda et al., 2008) and Russia (Demina, 2009, 2011; Ermakov, 2012) recognized the class independence, where syntaxonomic diversity of sandy steppes is higher than that of Central Europe. According to the authors, the leading factors of differentiation of communities in Festucetea vaginatae and Koelerio-Corynephoretea canescentis classes are the origin (genesis) of sandy substrates, as well as a reaction of soil solution. In the habitats of coenoses of the class Festucetea vaginatae, sandy soils were developed by marine and river sediments and have an alkaline reaction. Communities of Koelerio-Corynephoretea canescentis class were developed mainly on fluvioglacial sands having acidic reaction. This was confirmed by the ordination analysis results of alliances in these classes (Fig. 9). Climatic conditions of their distribution are also important in the steppe (Festucetea vaginatae) and forest and forest-steppe zones (Koelerio-Corynephoretea canescentis), respectively.

Ordination analysis of psammophytic vegetation alliances allowed us to determine the main factors of ecological differentiation of the communities (Fig. 9). They are primarily soil acidity (Rc) and salt regime (Sl). Most of the alliances are located along the DCA1 axis, i.e. along these vectors. According to soil $\mathrm{pH}$ gradient, coenoses of the classes Koelerio-Corynephoretea canescentis and Helichryso-Crucianelletea maritimae occupy the opposite positions. Communities of Festucetea vaginatae are located in the center, occupying an intermediate position and overlapping most of the HelichrysoCrucianelletea maritimae syntaxa, which indicates that they occur under similar environmental conditions. However, as the graph shows, one of the main parameters for the latter class is the content of carbonates $(\mathrm{Ca})$ and nitrogen $(\mathrm{Nt})$ in the soil. It is reasonable to assume an influence of ombroregime (Om) on distribution of Koelerio-Corynephoretea canescentis communities associated with forest and forest-steppe zones.

Syntaxa ratio in the classes Koelerio-Corynephoretea canescentis, Sedo-Scleranthetea (vegetation of rock outcrops) and Festucetea vaginatae also remains debatable. Individual European authors recognize the class Koelerio-Corynephoretea canescentis in a broad sense, combining all orders into a single class (Borhidi, 2003). Most researchers combine Koelerio-Corynephoretea canescentis and Sedo-Scleranthetea into one (Rodwell et al., 2002; Dengler, 2004; Chytrý, 2007; Sanda et al., 2008; Tzonev et al., 2009). In the latest review of European vegetation, Sedo-Scleranthetea was assigned to a separate class, and Festucetea vaginatae was included in Koelerio-Corynephoretea canescentis as an order (Mucina et al., 2016). Slovak (Valachovič et al., 1995; Jarolímek \& Šibík, 2008), Italian (Biondi et al., 2014), Spanish and Portuguese (RivasMartínez et al., 2001), Russian (Ermakov, 2012; Dulepova et al., 2018), as well as the majority of Ukrainian phytocoenologists maintain the position of the three classes being independent.

The syntaxonomic position of psammophytic communities with the participation of the shrub Salix rosmarinifolia (association Salici rosmarinifoliae-Holoschoenetum vulgaris Mititelu et al. 1973 and subassociation Centaureo brevicepsis-Festucetum beckeri salicetosum rosmarinifoliae Vicherek 1972) has to be clarified in the future. This is due to the fact that such coenoses were classified as Salicetea arenariae 
Weber 1999 on the Atlantic coast area of Western Europe (Mucina et al., 2016), but in Ukraine some authors classified it to the class NerioTamaricetea $\mathrm{Br}$-Bl. et O. de Bolòs 1958, the order Salicetalia arenariae Preising et Weber 1997, and to the provisionally allocated alliance Scirpoido holoschoeni-Salicion rosmarinifoliae I. Solomakha, Vorobyov et Moysienko 2015 prov. (Solomakha et al., 2015), and others classified it to the class Festucetea vaginatae (Dubyna et al., 2003, 2019a).

It should be noted that the position of Jasiono montanae-Festucetum ovinae Klika 1941 within Koelerion glancae is obviously somewhat ambiguous; since there is clearly a transitional position of its communities to meso-xerophytic vegetation on shell skeletal silicate soils of the SedoScleranthetea class. Czech scientists attributed this association to the alliance Hyperico perforati-Scleranthion perennis Moravec 1967 within the Sedo-Scleranthetea class (Sádlo \& Chytrý, 2007), but in Ukraine they are described on sandy habitats having a weak acidic reaction of soil solution; so, the authors previously assigned these communities to the KoelerioCorynephoretea canescentis class. Coenoflora composition and syntaxonomic position of the association Festuco psammophilae-Koelerietum glaucae sensu Gal'chenko 2006, non Klika 1931 remain unclear, since the diagnostic species given by the author was Festuca valesiaca (Galchenko, 2006), and the floristic composition differs significantly from the protolog. Further conducting of phytosociological studies of communities with the participation of such psammophytic coenose-forming species as Festuca psammophila and $F$. polesica are required, in particular, on the territory of the Ukrainian Polesie, which in this regard has not been sufficiently studied. Presumably, phytocoenoses of Festuco psammophilae-Koelerietum glaucae Klika 1931, Spergulo-Festucetum psammophilae Passarge 1960, Diantho arenarii-Festucetum polesicae R. Tx. 1937 and other associations should be distributed on the territory of Ukraine.

Probably, further research on communities in the little-known class Pyrolo-Pinetea sylvestris in Ukraine will allow us to determine the syntaxonomic position of associations Thymo pallasiani-Centauretum sumensis Shevchyk et Solomakha in Shevchyk, Solomakha et Voytuk 1996 and Cladonietum Shevchyk et Polishko 2000 nom. inval. (art. 3b) originally identified by the authors as part of Koelerion glaucae alliance (Shevchyk et al., 1996; Shevchyk \& Polishko, 2000). Previously, according to results obtained from the analysis of psammophytic vegetation, the associations in their floral composition were closer to native dry pine forests on sandy soils developed on the sandy terraces of large rivers (Koelerio glaucae-Pinion sylvestris alliance Ermakov 1999, Pyrolo-Pinetea sylvestris class).

Class Helichryso-Crucianelletea maritimae has been allocated recently on the territory of Ukraine (Dubyna et al., 2019a). Previously, its communities were considered within the class Festucetea vaginatae. There are currently two orders were allocated in Western Europe: Artemisio-Koelerietalia Sissingh 1974 (sandy meadows and shrubs on the rich stabilized grey dunes on the coasts of the Atlantic Ocean and of the Northern, the Ligurian and the Adriatic seas) and Crucianelletalia maritimae Sissingh 1974 (Mediterranean, Cantabro-Francoatlantic dwarf shrubs and Xerothermic meadows on stabilized coastal grey dunes). Their plant communities differ significantly from those of the Black Sea region; because of this a separate order was proposed in the class for the territory of Ukraine: Ephedro distachyaeMedicaginetalia romanicae (Dubyna et al., 2019a). Although some researchers see no rationale for separation of this class and consider it as a synonym of Ammophiletea (Marcenò et al., 2018), the results obtained allow us to maintain the position of its ecological and floral isolation. Despite the fact that both of these high-rank syntaxa include several common species (Eryngium maritimum, Leymus racemosus ssp. sabulosus, Ephedra distachya), the class Helichryso-Crucianelletea maritimae is characterized by the presence of a block of diagnostic species that distinguish it from the complex of diagnostic species Ammophiletea and Festucetea vaginatae, at least on the territory of Ukraine. These species are the following: Artemisia tschernieviana, Asparagus maritimus, Astragalus onobrychis, Centaurea apiculata ssp. adpressa, Erodium cicutarium, Medicago romanica $(\sim M$. sativa ssp. falcata), Melica transsilvanica ssp. klokovii, Scabiosa argentea, Tamarix gracilis, Teucrium polium, Trachomitum sarmatiense, Verbascum pinnatifidum. Ecologically stable dune communities also are distinct in that they do not experience periodic effects of sea waves, since they are protected by the berm crest, and their habitats have morphological differences. Most often, these are gently sloping uprush parts of berm crest and, to some extent, elevated sand deposits (dunes) anchored mostly by perennial vegetation. According to our data, communities of HelichrysoCrucianelletea maritimae class were even closer to Festucetea vaginatae than to Ammophiletea.

Worldwide, coastal dunes and other psammophytic habitats are recognized as highly vulnerable and dynamic ecosystems (Rannow \& Neubert, 2014; Acosta \& Ercole, 2015; Sarmati et al., 2019); in Ukraine and other countries they are systematically over-used. Inadequate land management contributes to the fact that these habitats are threatened with extinction, especially in the Mediterranean region (Sabatini et al., 2018). Frequent invasions of alien and atypical species into the structure of communities have been recorded; it resulted in their transformation and degradation (Dubyna et al., 2019b). All of this indicates the need for urgent actions to protect and preserve them.

\section{Conclusion}

Pioneer psammophytic vegetation of Ukraine is represented by 45 associations, 9 alliances and 3 orders belonging to 3 classes (KoelerioCorynephoretea canescentis, Helichryso-Crucianelletea maritimae and Festucetea vaginatae). The main factors of territorial and ecological differentiation of pioneer psammophytic vegetation in Ukraine were identified. It was found that the territorial distribution of communities of KoelerioCorynephoretea canescentis, Festucetea vaginatae and HelichrysoCrucianelletea maritimae were mainly influenced by ecotope mesorelief pattern, soil composition and the thickness of humic horizon, as well as the degree of aeolian processes development. The main factors of their ecological differentiation are soil acidity, salt content and ombroregime. Based on the results of DCA-ordination of syntaxa within certain vegetation classes, it was found that their distribution is influenced by factors that correlate with the environment-specific conditions. It was found that an ecological differentiation of syntaxa within Festucetea vaginatae is determined by the integrated effect of gradients, and soil salinity can be distinguished among these. The main factors in syntaxa distribution within the Koelerio-Corynephoretea canescentis class were temperature regime and climate continentality, as well as the gradients of ombroregime and soil humidity. The distribution of communities of Helichryso-Crucianelletea maritimae class in the ecological space is determined mainly by factors of variability of damping, ombroregime and climate continentality. Coenoses of Secaletum sylvestris, Diantho deltoidis-Armerietum elongatae, Corynephoro-Silenetum tataricae, Artemisio dniproicae-Sedetum sexangularis, Thymo angustifolii-Festucetum beckeri, Anisantho tectorum-Helichrysetum arenariae, Scabioso ucranicaeCaricetum ligericae and Ephedro-Caricetum colchicae associations were characterized by the widest ecological amplitude.

The authors adhere to the position of independence of the studied classes: Koelerio-Corynephoretea canescentis, Helichryso-Crucianelletea maritimae and Festucetea vaginatae, considering that the main factors of differentiation of communities in the classes Festucetea vaginatae and Koelerio-Corynephoretea canescentis were the origin (genesis) of sandy substrates, as well as the reaction of soil solution. Phytosociological analysis of a large number of coastal littoral vegetation relevés also provide support for independence of Helichryso-Crucianelletea maritimae and Ammophiletea classes based on their floristic and ecological differences.

The conducted research will allow the place of selected syntaxonomic units of the pioneer psammophytic vegetation of Ukraine to be determined in the pan-European system. The results of the work will be suitable for development of the Nature Reserve network and Eco-network of Ukraine, maintaining Prodrome of the vegetation of Ukraine, Prodrome of the vegetation of Europe, preparing the next volume of the publication "Vegetation of Ukraine" and "Green Data Book of Ukraine", for further study of successional and adaptation processes in extreme environmental conditions of pioneer habitats, establishing patterns of regenerative geosystems' development and finding out the ways to manage them. The ability of plant communities to indicate the environment state (phytoindication of environmental conditions), which in recent years has become an urgent sociological necessity, will allow the environmental situation in the regions to be monitored, as well help in as determining trends in their further changes.

The authors gratefully acknowledge A. Kuzemko, O. Kovalenko, O. Chusova, O. Senchylo, V. Kolomiychuk, T. Fitsailo, I. Goncharenko, V. Konischuk, G. 
Tsukanova, Y. Gaiova, D. Iakushenko, V. Konogray, for the kindly provided relevés of psammophyte vegetation from their dissertation manuscripts.

\section{References}

Acosta, A., \& Ercole, S. (Eds.). (2015). Gli habitat delle coste sabbiose italiane: Ecologia e problematiche di conservazione [Italian coastal dune habitats: Ecology and conservation issues]. ISPRA, Serie Rapporti (in Italian).

Aleshkina, U. M. (2011). Rastitelnye soobshchestva zelenoy zony g. Kieva [Plant communities of the green zone of Kiev]. In: Vserossiyskaya nauchnaya konferentsiya "Otechestvennaya geobotanika: Osnovnye vekhi i perspektivy". Boston-Spektr, Sankt-Petersburg. Pp. 7-10 (in Russian).

Androsova, A. Y., \& Solomakha, T. D. (1996). Psamofilna roslynnist' Bilosarayskoi kosy i morskoho uzberezhzhya poblyzu m. Mariupolya [Psammophilous vegetation of the Bilosarai spit and the sea coast near Mariupol]. Ukrainian Phytosociological Collection, Series A, 1, 41-49 (in Ukrainian).

Bairak, O. M. (1998). Florystychna klasyfikatsiya roslynnoho pokryvu Livoberezhnoho Prydniprov'ya [Floristic classification of vegetation of the Left Bank Dnieper]. Ukrainian Botanical Journal, 55(2), 139-145 (in Ukrainian)

Bardat, J., Bioret, F., Botineau, M., Boullet, V., Delpech, R., Genu, J.-M., Haury, J. Lacoste, A., Rameau, J.-C., Royer, J.-M., Roux, G., \& Touffet, J. M. (2004). Prodrome des végétations de France [The prodrome of French vegetation]. Muséum National d'Histoire Naturelle, Paris (in French).

Berg, C., Dengler, J., Abdank, A., \& Iermann, M. (Eds.). (2004). Die Pflanzengesellschaften Mecklenburg-Vorpommerns und ihre Gefährdung [The plant communities of Mecklenburg-Vorpommern and their vulnerability]. Wiessdorn Verlag, Jena (in German).

Biondi, E., Blasi, C., Allegrezza, M., Anzellotti, I., Azzella, M. M., Carli, E., Casavecchia, S., Copiz, R., Del Vico, E., Facioni, L., Galdenzi, D., Gasparri, R., Lasen, C., Pesaresi, S., Poldini, L., Sburlino, G., Taffetani, F., Vagge, I., \& Zivkovic, L. (2014). Plant communities of Italy: The vegetation prodrome. Plant Biosystems, 148(4), 728-814.

Bondareva, V. V., Nikolaychuk, L. F., \& Golub, V. B. (2019). Kserofitnye rastitelnye soobshchestva doliny Nizhney Volgi [Xerophytic plant communities of the Lower Volga valley]. Phytodiversity of Eastem Europe, 13(1), 4-29 (in Russian).

Borhidi, A. (2003). Magyarorszag növćnytársulásai [Plant associations in Hungary] Akadémiai Kiadó, Budapest (in Hungarian).

Braun-Blanquet, J. (1964). Pflanzensoziologie - Grundzüge der Vegetationskunde [Phytosociology - Broad Vegetation Science]. 3rd ed. Springer, Wien (in German).

Bulokhov, A. D. (2019). Soobshchestva klassa Sedo-Scleranhetea Br.-Bl. 1955 v poyme reki Desny [Communities of the class Sedo-Scleranhetea Br.-Bl. 1955 in floodplain of the Desna River]. Diversity of Plant World B, 3(3), 56-66 (in Russian)

Cao, G., Tsuchiya, K., Zhu, W., \& Okuro, T. (2019). Vegetation dynamics of abandoned paddy fields and surrounding wetlands in the lower Tumen River Basin Northeast China. PeerJ, 7, 1-17.

Chusova, O. O. (2019). Roslynnist' ta biotopy baseynu r. Krasna [Vegetation and biotopes of the Krasna river basin]. Phytosociocentre, Kyiv (in Ukrainian).

Chynkina, T. B. (2003). Syntaksonomiya i antropohenna dynamika roslynnosti hyrlovoji oblasti Dnipra [Syntaxonomy and anthropogenic dynamics of vegetation of the mouth of the Dnieper]. Publishing Kherson National University, Kyiv (in Ukrainian).

Chytrý, M. (Ed.). (2007). Vegetace České republiky. 1. Travinná a keřičková vegetace [Vegetation of the Czech Republic. 1. Grassland and Heathland Vegetation]. Academia, Praha (in Czech)

Chytrý, M., Chiarucci, A., Pärtel, M., Pillar, V. D., Bakker, J. P., Mucina, L., Peet, R. K., \& White P. S. (2019). Progress in vegetation science: Trends over the past three decades and new horizons. Journal of Vegetation Science, 31(1), 1-4.

Çoban, S., \& Willner, W. (2019). Numerical classification of the forest vegetation in the Western Euxine Region of Turkey. Phytocoenologia, 49 (1), 71-106.

Davydova, A. O. (2019). Syntaksonomiya roslynnosti Natsionalnoho Pryrodnoho Parku "Dzharylhatskyi". Klas Festucetea vaginatae [Syntaxonomy of vegetation of national nature park "Dzharylhatskyi". The class Festucetea vaginatae] Biology and Ecology, 5(1), $34-43$ (in Ukrainian).

Demina, O. N. (2009). Soobshchestva klassa Festucetea vaginatae Soó em. Vicherek 1972 na territorii Tsimlyanskikh peskov v Rostovskoy oblasti [Communities of the class Festucetea vaginatae Soó em. Vicherek 1972 on the territory of Tsimlyansk sands in the Rostov region]. In: Materialy Moskovskogo Tsentra Russkogo Geograficheskogo Obshchestva. Biogeografiya, Moscow, 15, 27-38 (in Russian).

Demina, O. N. (2015). Klassifikatsiya rastitelnosti stepey basseyna Dona [Classification of the steppe vegetation of the Don basin]. Publisher of the Southern Federal University, Rostov na Donu (in Russian).

Dengler, J. (2004). Klasse Koelerio-Corynephoretea Klika in Klika et Novak 1941 Sandtrockenrasen und Felsgrusfluren von der submeridionalen bis zur borealen Zone [Class Koelerio-Corynephoretea Klika in Klika et Novak 1941 - dry sand turf and rocky meadows from the submeridional to the boreal zone]. In: Berg, C., Dengler, J., Abdank, A., \& Iermann, M. (Eds.). Die Pflanzengesellschaften Mecklenburg-Vorpommerns und ihre Gefährdung. Wiessdorn Verlag, Jena. Pp. 301-326 (in German).

Didukh, Y. P. (2011). The ecological scales for the species of Ukrainian flora and their use in synphytoindication. Phytosociocentre, Kyiv.

Didukh, Y. P. (2012). Osnovy bioindykatsii [Fundamentals of bioindication] Naukova Dumka, Kyiv (in Ukrainian)

Didukh, Y. P., \& Korotchenko, I. A. (1996). Stepova roslynnist' pivdennoi chastyny Livoberezhnoho Lisostepu Ukrainy. 1: Klasy Festucetea vaginatae ta Helianthemo-Thymetea [Steppe vegetation of the southem part of the Left-Bank Forest-Steppe of Ukraine. 1: Classes Festucetea vaginatae and HelianthemoThymetea]. Ukrainian Phytosociological Collection, Series A, 2, 56-63 (in Ukrainian).

Dring, J., Hoda, P., Mersinllari, M., Mullaj, A., Pignatti, S., \& Rodwell, J. (2002). Plant communities of Albania - a preliminary overview. Annali di Botanica, 2 , 7-30.

Dubyna, D. V., Neuhäuslová, Z., \& Shelyag-Sosonko, Y. R. (1995). Vegetation of the "Birjucij Island" Spit in Azov Sea. Sand Steppe Vegetation. Folia Geobotanica et Phytotaxonomica, 30, 1-31.

Dubyna, D. V., Dziuba, T. P., Zhmud, O. I., Tymoshenko, P. A., \& Shelyag-Sosonko, Y. R. (1996). Syntaksonomiya roslynnosti Zhebryyanskoho prymorskoho pasma (Odeska obl.). I. Pisky [Syntaxonomy of vegetation of the Zhebriyansky coastal ridge (Odessa region). I. Sands]. Ukrainian Phytosociological Collection, Series A, 2, 44-56 (in Ukrainian).

Dubyna, D. V., Shelyag-Sosonko, Y. R., Zhmud, O. I., Dvoretskiy, T. V., Dziuba, T. P., \& Tymoshenko, P. A. (2003). Dunayskyi biosfernyi zapovidnyk. Roslynnyi svit [Dunaisky Biosphere Reserve. Plant Kingdom]. Phytosociocentre, Kyiv (in Ukrainian).

Dubyna, D. V., Neuhäuslová, Z., Dziuba, T. P., \& Shelyag-Sosonko, Y. R. (2004). Klasyfikatsiya ta prodromus syntaksonomichnoi riznomanitnosti vodoym, perezvolozhenykh terytoriy ta aren Pivnichnoho Prychornomor'ya [Classification and Prodrome of vegetation of reservoirs, overmoisturized territories and aren of the Nothern part of the Black Sea region]. Phytosociocentre, Kyiv (in Ukrainian)

Dubyna, D. V., \& Dziuba, T. P. (2005). Fitotsenotychna riznomanitnist' ostrova Dzharylhach (Khersonska oblast) [Phytocenotic diversity of Dzharylgach island (Kherson region)]. Ukrainian Botanical Journal, 62(2), 255-269 (in Ukrainian).

Dubyna D. V. Tymoshenko, P. A \& \& Dvoretskyi, T. V. (2009). Ekoloho-florystychni osoblyvosti uhrupovan klasu Festucetea vaginatae v Ukraini ta zavdannya yikh okhorony [Ecological and floristic features of groups of the class Festucetea vaginatae in Ukraine and tasks of their protection] Chornomorski Botanical Journal, 5(4), 491-501 (in Ukrainian).

Dubyna, D. V., Dziuba, T. P., Iemelianova, S. M., Bagrikova, N. O., Borysova O. V., Borsukevych, L. M., Vynokurov, D. S., Gapon, S. V., Gapon, Y. V., Davydov, D. A., Dvoretsky, T. V., Didukh, Y. P., Zhmud, O. I., Kozyr, M. S., Konyschuk, V. V., Kuzemko, A. A., Paskevych, N. A., Ryff, L. E., Solomakha, V. A., Felbaba-Klushyna, L. M., Fitsailo, T. V., Chorna, G. A., Chorney, I. I., Shelyag-Sosonko, Y. R., \& Iakushenko, D. M. (2019a). Prodromus roslynnosti Ukrainy [Prodrome of the vegetation of Ukraine]. Naukova Dumka, Kyiv (in Ukrainian).

Dubyna, D. V., Iemelianova, S. M., Dvoretzkiy, T. V., Dziuba, T. P., \& Tymoshenko, P. A. (2019b). Adventization of coenofloras of the classes of pioneer vegetation in Ukraine. Ukrainian Botanical Journal, 76(6), 499-510 (in Ukrainian).

Dulepova, N. A., Korolyuk, A. Y., Yamalov, S. M., Lebedeva, M. V., \& Golovanov, Y. M. (2018). Rastitelnost peschanykh stepey Orenburgskoy oblasti [Sandy steppe vegetation in Orenburg Region]. Vegetation of Russia, 33 , 53-65 (in Russian)

Dziuba, T. P., Solomakha, T. D., \& Tymoshenko, P. A. (2010). Roslynni uhrupovannya [Vegetation communities]. In: Pereyaslav-Khmelnytskiy. Nature: Vegetation. Critical inventory annotated notes of flora and vegetation: Vascular plants, bryophytes, lichens, and algae. FOP Majdanchenko I. S., KorsunShevchenkivsky, 86-95 (in Ukrainian).

Erdős, L., Ambarl, D., Anenkhonov, O. A., Bátori, Z., Cserhalmi, D., Kiss, M., Kröel-Dulay, G., Liu, H., Magnes, M., Molnár, Z., Naqinezhad, A. Semenishchenkov, Y. A., Tölgyesi, C., \& Török, P. (2018). The edge of two worlds: A new review and synthesis on Eurasian forest-steppes. Applied Vegetation Science, 21, 345-362.

Ermakov, N. B. (2012). Prodromus vysshikh edinits rastitelnosti Rossii [Prodrome of higher units of vegetation in Russia]. In: Mirkin, B. M., \& Naumova, L. G. (Eds.). Sovremennoe sostoyanie osnovnykh kontseptsiy nauki o rastitelnosti [The current state of the basic concepts of vegetation science]. Gilem, Ufa. Pp. 377-483 (in Russian).

Gaiova, J. Y. (2015). Psamofitna roslynnist' Cherkasko-Chyhyrynskoho heobotanichnoho rayonu [The Psamofit Vegetation of Cherkasy-Chigirin Geobotanic Region]. Naukovyi Visnyk NLTU Ukrainy, 25(9), 131-138 (in Ukrainian). 
Gomlya, L. M. (2005). Roslynnist' dolyny richky Khorol [Vegetation of the Khorol River valley]. Ukrainian Phytosociological Collection, Series A, 22, 2-187 (in Ukrainian).

Gordienko, I. I. (1969). Oleshskie peski i biogeotsenoticheskie svyazi v protsesse ikh zarastaniya [Oleshski Sands and biogeocenotic connections in the process of their overgrowth]. Naukova Dumka, Kyiv (in Ukrainian).

Galchenko, N. P. (2006). Rehionalnyi landshaftnyi park "Kremenchutski plavni". Roslynnyi svit [Kremenchuk Plavni Regional Landscape Park. Plant Kingdom]. Phytosociocentre, Kyiv (in Ukrainian).

Hennekens, S. M., \& Schaminée, J. H. J. (2001). Turboveg, a comprehensive date base management system for vegetation data. Journal of Vegetation Science, 12,589-591.

Hill, M. O., \& Gauch, H. G. (1980). Detrended correspondence analysis: An improved ordination technique. Vegetatio, 42, 47-58.

Hill, M. O. (1979). Twinspan - a Fortran program for arranging multivariate data in an ordered two-way table by classification of the individuals and the attributes. Ithaca, NY.

Jansons, Ā., Robalte, L., Čakšs, R., \& Matisons, R. (2016). Long-term effect of whole tree biomass harvesting on ground cover vegetation in a dry Scots pine stand. Silva Fennica, 50(5), 1661.

Jarolímek, I., Šibík, J., Hegedüšová, K., Janišová, M., Kliment, J., Kučera, P., Májeková, J., Michálková, D., Sadloňová, J., Šibíková, I., Škodová, I., Tichý, L., Ujházy, K., Ujházyová, M., Uhliirová, J., Valachovič, M., \& Zaliberová, M. (2008). Diagnostic, constant and dominant species of the higher vegetation units of Slovakia. Veda, Bratislava.

Konishchuk, V. V. (2006). Otsinka riznomanitnosti ekosystem Cheremskoho Pryrodnoho Zapovidnyka na osnovi kartohrafichnoho modelyuvannya [Estimation of ecosystem diversity of Cheremsky Nature Reserve on the basis of cartographic modeling]. Dia, Kyiv (in Ukrainian).

Korolyuk, A. Y., Lebedeva, M. V., Yamalov, S. M., Golovanov, Y. M., Dulepova, N. A., Zolotareva, N. V., \& Teptina, A. Y. (2018). The petrophytic steppes of the Urals: Diversity and ecological drivers. The Fourth International scientific conference on ecology and geography of plants and plant communities. $\mathrm{KnE}$ Life Sciences. Pp. 88-94.

Korzhenevsky, V. V. (1986). Rastitelnost dyun Kryma [The vegetation of the Crimean dunes]. Collection of Scientific Papers of the State Nikitsky Botanical Garden, 98, 122-133 (in Russian).

Korzhenevsky, V. V., \& Klyukin, A. A. (1990). Rastitelnost abrazionnykh i akkumulyativnykh form rel'efa morskikh poberezhiy i ozer Kryma [Vegetation of abrasion and accumulative relief forms of the Crimean sea coasts and lakes] Moscow, Dep. v VINITI 10.07.1990, No 3822-B90 (in Russian).

Korzhenevsky, V. V., Bagrikova, N. A., Ryff, L. E., \& Levon, A. R. (2003). Prodromus rastitelnosti Kryma (20 let na platforme floristicheskoy klassifikatsii) [Prodromus of the Crimean vegetation (20 years on the platform of floristic classification)]. Bulletin of Main Botanical Garden of RAN, 186, 32-51 (in Russian).

Korzhenevsky, V. V., \& Kvitnitskaya, A. A. (2014). Sintaksonomiya rastitelnosti eolovogo relefa Kryma [Syntaxonomy of vegetation of the Crimean eolian relief] Works of the State Nikitsky Botanical Gardens, 136, 41-55 (in Russian).

Kovalenko, O. A. (2016). Flora, roslynnist' ta fitosozolohichni aspekty NPP "Pyryatynskyi" [Flora, vegetation and phytososological aspects of NPP "Pyriatynsky"]. Mria, Sumy (in Ukrainian).

Kuzemko, A. (2009). Dry grasslands on sandy soils in the forest and forest-steppe zones of the plains region of Ukraine: Present state of syntaxonomy. Tuexenia, $29,369-390$

Kuzemko, A., Becker, T., Didukh, Y. P., Arde-lean, I. V., Becker, U., Beldean, M., Dolnik, C., Jeschke, M., Naqinezhad, A., Uğurlu, E., Ünal, A., Vassilev, K. Vorona, E. I., Yavorska, O. H., \& Dengler, J. (2014). Dry grassland vegetation of Central Podolia (Ukraine) - a preliminary overview of its syntaxonomy, ecology and biodiversity. Tuexenia, 34, 391-430.

Kuzemko, A. A., Steinbauer, M. J., Becker, T., Didukh, Y. P., Dolnik, C., Jeschke, M., Naqinezhad, A., Ugurlu, E., Vassilev, K., \& Dengler, J. (2016). Patterns and drivers of phytodiversity of steppe grasslands of Central Podolia (Ukraine). Biodiversity and Conservation, 25(12), 2233-2250.

Landucci, F., Šumberová, K., Tichý, L., Hennekens, S., Aunina, L., Biță-Nicolae, C., Borsukevych, L., Bobrov, A., Čarni, A., De Bie, E., Golub, V., Hrivnák, R., Iemelianova, S., Jandt, U., Jansen, F., Kacki, Z., Lájer, K., Papastergiadou, E., Šilc, U., Sinkevičienè, Z., Stančić, Z., Stepanovič, J., Teteryuk, B., Tzonev, R., Venanzoni, R., Zelnik, I., \& Chytrý, M. (2020). Classification of the European marsh vegetation (Phragmito-Magnocaricetea) to the association level. Applied Vegetation Science, 23 (2), 297-316

Lengyel, A., Landucci, F., Mucina, L., Tsakalos, J. L., \& Botta-Dukát, Z. (2018). Joint optimization of cluster number and abundance transformation for obtaining effective vegetation classifications. Journal of Vegetation Science, 29, 336-347.

Marcenò, C., Guarino, R., Loidi, J., Herrera, M., Isermann, M., Knollová, I., Tichý, L., Tzonev, R. T., Acosta, A. T. R., FitzPatrick, Ú., Iakushenko, D., Janssen, J. A. M., Jiménez-Alfaro, B., Kącki, Z., Keizer-Sedláková, I., Kolomiychuk,
V.P., Rodwell, J. S., Schaminée, J. H. J., Šilc, U., \& Chytrý, M. (2018). Classification of European and Mediterranean coastal dune vegetation. Applied Vegetation Science, 21(1), 1-27.

Matuszkiewicz, W. (2008). Przewodnik do oznaczania zbiorowisk roślinnych Polski [Guide for identification of the plant communities of Poland]. Państwowe Wydawnictwo Naukowe, Warszawa (in Polish).

Mucina, L., Bültman, H., Dierssen, K., Theurillat, J.-P., Dengler, J., Čarni, A., Šumberová, K., Raus, T., Di Pietro, R., Gavilán Garcia, R., Chytrý, M., Iakushenko, D., Schaminée, J. H. J., Bergmeier, E., Santos Guerra, A., Daniëls, F. J.A., Ermakov, N., Valachovič, M., Pigantti, S., Rodwell, J. S., Pallas, J., Capelo, J., Weber, H. E., Lysenko, T., Solomeshch, A., Dimopoulos, P., Aguiar, C., Freitag, H., Hennekens, S. M., \& Tichý, L. (2016). Vegetation of Europe: Hierarchical floristic classification system of vascular plant, bryophyte, lichen, and algal communities. Applied Vegetation Science, 19(1), 3-264.

Onyshchenko, V. A. (2006). Florystychna klasyfikatsiya roslynnosti Ukrainskoho Polissya [Floristic classification of vegetation of Ukrainian Polissya]. In: Fitoriznomanittya Ukrainskoho Polissya ta yoho okhorona [Phytodiversity of Ukrainian Polissya and its protection]. Phytosociocentre, Kyiv. Pp. 43-84 (in Ukrainian).

Orlov, O. O., \& Yakushenko, D. M. (2005). Roslynnyi pokryv proektovanoho Korostyshivskoho natsionalnoho pryrodnoho parku [Vegetation of the projected Korostyshiv National Nature Park]. Phytosociocentre, Kyiv (in Ukrainian).

Polishko, O. D. (2001). Syntaksonomiya roslynnosti dilyanky borovoi terasy (Prokhorivske lisnytstvo Cherkaskoi oblasti) [Syntaxonomy of pine terrace vegetation (Prochoriv forestry of Cherkasy region)]. Zapovidna Sprava v Ukraini, 7(1), 11-20 (in Ukrainian).

Polishko, O. D. (2005). Syntaksonomiya roslynnosti dilyanky borovoi terasy Dnipra (Chyhyrynske lisnytstvo Cherkaskoi oblasti) [Syntaxonomy of vegetation of the Dnieper pine terrace area (Chyhyryn forestry of Cherkasy region)]. Aktualni Problemy Botaniky ta Ekolohii, 1, 163-176 (in Ukrainian)

Rannow, S., \& Neubert, M. (Eds.). (2014). Managing protected areas in Central and Eastern Europe under climate change. Advances in Global Change Research. Vol. 58. Springer Nature.

Rivas-Martínez, S., Fernández-González, F., Loidi, J., Lousã, M., \& Penas, A (2001). Syntaxonomical checklist of vascular plant communities of Spain and Portugal to association level. Itinera Geobotanica, 14, 5-341.

Rodwell, J. S., Schaminee, J. H. J., Mucina, L., Pignatti, S., Dring, J., \& Moss, D. (2002). The diversity of European vegetation. An overview of phytosociological alliances and their relationships to EUNIS habitats. National Centre for Agriculture, Nature Management and Fisheries, Wageningen.

Roleček, J., Tichý, L., Zelený, D., \& Chytrý, M. (2009). Modified Twinspan classification in which the hierarchy respects cluster heterogeneity. Joumal of Vegetation Science, 20, 596-602.

Sabatini, F. M., Jiménez-Alfaro, B., Burrascano, S., Lora, A., Chytrý, M. (2018). Beta-diversity of Central European forests decreases along an elevational gradient due to the variation in local community assembly processes. Ecography, 41 $1038-1048$.

Sádlo, J., \& Chytrý, M. (2007). Jasiono montanae-Festucetum ovinae Klika 1941. In: Chytrý M. (Ed.), Vegetace České republiky. 1. Travinná a keřǐčková vegetace [Vegetation of the Czech Republic. 1. Grassland and Heathland Vegetation]. Pp. 353-355 (in Czech).

Sanda, V., Öllerer, K., \& Burescu, P. (2008). Fitocenozele din Romănia. Sintaxonomia, structură, dinamică si evolutie. Ars Docendi, Bucureşti (in Romanian).

Sarmati, S., Bonari, G., \& Angiolini, C. (2019). Conservation status of Mediterranean coastal dune habitats: Anthropogenic disturbance may hamper habitat assignment. Rendiconti Lincei. Scienze Fisiche e Naturali, 30, 623-636.

Senchylo, O. O. (2010). Roslynnist' zaplavy Dnipra v mezhakh Lisostepu [Vegetation of the Dnieper floodplain within the Forest-Steppe zone]. Phytosociocentre, Kyiv (in Ukrainian)

Shevchyk, V. L., \& Polishko, O. D. (2000). Syntaksonomiya roslynnosti dilyanky borovoi terasy Dnipra (Liplyavske lisnytstvo Cherkaskoi oblasti) [Syntaxonomy of pine terrace vegetation (Liplavo forestry of Cherkasy region)] Ukrainian Phytosociological Collection, Series A, 16, 67-89 (in Ukrainian).

Shevchyk, V. L., \& Solomakha, V. A. (1996). Syntaksonomiya roslynnosti ostroviv Kruhlyk ta Shelestiv Kanivskoho pryrodnoho zapovidnyka [The syntaxonomy of vegetation of Kruglyk and Shelestiv islands in Kaniv Nature Reserve]. Ukrainian Phytosociological Collection, Series A, 1, 12-27 (in Ukrainian).

Shevchyk, V. L., Solomakha, V. A., \&Voityuk, Y. O. (1996). Syntaksonomiya roslynnosti ta spysok flory Kanivskoho pryrodnoho zapovidnyka [The syntaxonomy of vegetation and list of the flora of Kaniv Nature Reserve]. Ukrainian Phytosociological Collection, Series B, 1(4), 2-120 (in Ukrainian).

Slezák, M., Hrivnák, R., \& Machava, J. (2017). Environmental controls of plant species richness and species composition in black alder floodplain forests of central Slovakia. Tuexenia, 37, 79-94.

Solomakha, I. V., Vorobyov, Y. O., \& Moysiyenko, I. I. (2015). Roslynnyi pokryv lisiv ta chaharnykiv Pivnichnoho Prychornomor'ya [Vegetation of forests 
and shrubs of the Northern Black Sea Coast]. Phytosociocentre, Kyiv (in Ukrainian)

Solomakha, V. A. (2008). Syntaksonomia rosnynnosti Ukrainy. Tretie nablyzhennia [The syntaxonomy of vegetation of the Ukraine. The third approximation] Phytosociocentre, Kyiv (in Ukrainian).

Sörensen, T. A. (1948). A method of establishing groups of equal amplitude in plant sociology based on similary of species content, and its application to analyses of the vegetation on Danish commons. Kongelige Danske Videnskabernes Selskabs Biologiske Skrifter, 5, 1-34.

Soroka, M. I. (2008). Roslynnist' Ukrainskoho Roztochchya [Vegetation of the Ukrainian Roztocze]. Svit, Lviv (in Ukrainian).

Ter Braak, C. J. F., \& Smilauer, P. (2015). Topics in constrained and unconstrained ordination. Plant Ecology, 216(5), 683-696.

Tichy, L. (2002). Juice, software for vegetation classification. Journal of Vegetation Science, $13,451-453$.

Tsukanova, H. O. (2005). Florystychne ta tsenotychne riznomanittya ostroviv Dnipra $\mathrm{v}$ mezhakh $\mathrm{m}$. Kyyeva ta yoho okhorona [Floristic and coenotic diversity of the Dnieper islands within the city of Kyiv and its protection]. Kyiv (in Ukrainian).

Tymoshenko, P. A. (1999). Suchasnyi stan klasyfikatsii roslynnosti aren pivdnya Ukrainy [The modern state of classification of vegetation of the arenas of Southern Ukraine]. Ukrainian Phytosociological Collection, Series A, 12-13, 201-205 (in Ukrainian).

Tyshchenko, O. V. (2006). Roslynnist' prymorskykh kis pivnichnoho uzberezhzhya Azovskoho morya [The vegetation of the northern coastal spits of the Sea of Azov]. Phytosociocentre, Kyiv (in Ukrainian).

Tzonev, R. T., Dimitrov, M. A., \& Roussakova, V. H. (2009). Syntaxa according to the Braun-Blanquet approach in Bulgaria. Phytologia Balcanica, 15(2), 209-233.

Umanets, O. Y., \& Solomakha, I. V. (1999a). Syntaksonomiya roslynnosti Chornomorskoho biosfernoho zapovidnyka. II. Ostriv Tendra [Syntaxonomy of vegetation of the Black Sea Biosphere Reserve. II. Tendra Island]. Ukrainian Phytosociological Collection, Series A, 12-13, 63-77 (in Ukrainian).

Umanets, O. Y., \& Solomakha, I. V. (1999b). Syntaksonomiya roslynnosti Chornomorskoho biosfernoho zapovidnyka. III. Dilyanka Ivano-Rybalchanska [Syntaxonomy of vegetation of the Black Sea Biosphere Reserve. III. IvanoRybalchanska site]. Ukrainian Phytosociological Collection, Series A, 14, 84 102 (in Ukrainian).

Valachovič, M., Ot’ahel'ová, H., Stanová, V., \& Maglocký, Š. (Eds.). (1995). Rastlinné spoločenstvá Slovenska 1. Pionierska vegetácia [Plant communities of Slovakia 1. Pioneer vegetation]. Veda, Bratislava.
Venables, W. N., \& Smith, D. M. (2008). An introduction to R. 2nd edition. Network Theory Ltd.

Vicherek, J. (1972). Die Sandpflanzengesselschaften des unteren und mittleren Dnieprstromgebietes (die Ukraine). Folia Geobotanica et Phytotaxonomica, 7, $9-46$.

Vorobyov, Y. O., Balashov, L. S., \& Solomakha, V. A. (1997). Syntaksonomiya roslynnosti Polis'kogo pryrodnogo zapovidnyka [The syntaxonomy of vegetation of the Polesie Nature Reserve]. Ukrainian Phytosociological Collection, Series B, 8, 2-128 (in Ukrainian).

Ward, J. H., Jr. (1963). Hierarchical grouping to optimize an objective function. Journal of the American Statistical Association, 58, 236-244.

Weber, H. E., Moravec, J., \& Theurillat, J.-P. (2000). International code of phytosociological nomenclature. 3rd edition. Journal of Vegetation Science, $11,739-768$.

Westhoff, V., \& van der Maarel, E. (1973). The Braun-Blanquet approach. 2-nd ed. In: Whittaker, R. (Ed.). Classification of Plant Communities. Pp. 287-399.

Whittaker, R. H. (1978). Approaches to classifying vegetation on classification of plant communities. 2-nd ed. The Hague, Junk.

Willner, W., Roleček, J., Korolyuk, A., Dengler, J., Chytrý, M., Janišová, M., Lengyel, A., Aćić, S., Becker, T., Ćuk, M., Demina, O., Jandt, U., Kącki, Z., Kuzemko, A., Kropf, M., Lebedeva, M., Semenishchenkov, Y., Šilc, U., Stančić, Z., Staudinger, M., Vassilev K., \& Yamalov, S. (2019). Formalized classification of semi-dry grasslands in Central and Eastern Europe. Preslia, 91, $25-49$.

Willner, W., Tichy, L., \& Chytry, M. (2009). Effects of different fidelity measures and contexts on the determination of diagnostic species. Joumal of Vegetation Science, 20, 130-137.

Yakushenko, D. M. (2004). Nova asotsiatsiya psamofilnoi roslynnosti zi skhodu Zhytomyrskoho Polissya [New association of psammophilous vegetation from the east of Zhytomyr Polissya]. Visnyk of Lviv National University. Biology Series, 35, 95-101 (in Ukrainian).

Yousaf, A., Shabbir, R., Jabeen, A., Erum, S., \& Ahmad, S. S. (2016). Linkage between herbaceous vegetation and soil characteristics along rawal dam Islamabad. Journal of Soil Science and Plant Nutrition, 16(1), 88-100.

Zhou, L., Shen, H., Chen, L., Li, H., Zhang, P., Zhao, X., Liu, T., Liu, Sh., Xing, A., Hu, H., \& Fang, J. (2019). Species richness and composition of shrubencroached grasslands in relation to environmental factors in northern China. Journal of Plant Ecology, 12(1), 56-66. 